\title{
GENERATION PRINCIPLES FOR ARM MOVEMENTS IN HUMANS
}

\author{
Dissertation \\ ZUR ERLANGUNG DES MATHEMATISCH-NATURWISSENSCHAFTLICHEN \\ DoKTORgrades "DoKTOR RERUm NATURALIUM" \\ Der Georg-August-Universität Göttingen
}

vorgelegt von

Katja Fiedler

aus Wurzen

Göttingen 2011 
Prof. Dr. Theo Geisel (Referent)

Abteilung für Nichtlineare Dynamik, MPIDS

Institut für Nichtlineare Dynamik, Fakultät für Physik

Prof. Dr. Florentin Wörgötter (Koreferent)

III. Physikalisches Institut, Fakultät für Physik

Dr. Michael Herrmann

IPAB, School of Informatics, Edinburgh

Tag der Verteidigung: 17.06.2011 


\section{Affidavit}

I hereby declare that this dissertation was written independently and with no other sources and aids than quoted.

\section{Feidl}

Göttingen, April 29th, 2011

Katja Fiedler

\section{Acknowledgements}

First of all, I would like to show my gratitude to my thesis committee - Theo Geisel, Florentin Wörgötter, and Michael Herrmann. Without their constant support this thesis would not have been possible.

Furthermore, I would like to thank Annette Witt, Tamar Flash, Sara Solla, Martha Flanders, Anatol Feldman, and Mark Latash for their support and many helpful discussions. My thanks also go to the interesting people I met at the nld.ds.mpg and in the Wörgötter group at Physics department. I also owe my deep gratitude to all the people who made a $\mathrm{PhD}$ students life easier thanks to Regina, Ursel, Tanja, Katharina, Barbara, Yorck, and so many more. I would also like to thank my parents for their patience and support, Marcus, Lishma and Fabio for the great time we had together, Arndt for his constant support and many helpful conversations, my friends from the kayak team of the Waspo Göttingen for the valuable recreation, and, last but not least, Marco for his encouraging distractions. 



\section{Abstract}

This thesis comprises studies on the two major domains of motor control posture and movement - with special focus on human upper limbs.

Endeffector stiffness characterises the resistance of the arm against external disturbances in the hand coordinate frame, and is the key quantity to study arm posture. This thesis presents a method for stiffness estimation in threedimensional endeffector space. The approach enables us to study stiffness in natural postures throughout the daily used workspace. Derived stiffness is tested in the light of results known from classical studies on human upper limbs restricted to a horizontal plane. A variation of stiffness direction and size with hand position as well as with arm posture is found. While the direction of highest stiffness is shown to be aligned with the hand-shoulder axis in planar studies (cf. Flash and Mussa-Ivaldi, 1990), it turns out to be approximately in a line with the forearm axis in the unrestrained arm. This suggests that major compensations of external disturbances are accomplished at shoulder level.

The second part of this thesis deals with human arm movements under time pressure in left- as well as right-handed participants using both preferred and non-preferred hand. A framework to set up virtual parcours to asses via-point movements in combination with an obstacle avoidance task has been developed. Hand trajectories were analysed with respect to dynamic characteristics. Furthermore, an uncertainty about the movement task was imposed by revealing the final movement target just when the participants had to choose on which side - either left or right - to pass the obstacle. Using this bottleneck of processing the new information about the task and its transformation into a motor command allows to study the participants' decision behaviour.

It turns out that with increasing time pressure - or better: rush in execution - attention is more and more (with)drawn from sensory input towards a focus on the own behavioural pattern. Participants who show fast execution of the trials also show a strong stereotypisation of movement direction choice. If an initial overrepresentation of movement targets on the right side was presented, the preferred direction always coincided with it. For overemphasation of left target locations, not all participants preferred this direction, but some the opposite one. These phenomena were rather independent of handedness and

used hand. One possible explanation could be found in cultural imprinting of rightwards movements in reading, time lines, and also visual communication. A direct response to the presented target locations can be excluded on several 
time scales, especially in the fast participants. Behaviour is shown to mainly depend on the own behavioural history. Furthermore, stereotypisation increased with increasing movement speed. This heuristic that saves computation time in critical situations and the organisation of subsequent error corrections can serve as an inspiration for the design of flexible but fast robot controllers. 


\section{Contents}

1 Introduction 1

1.1 Motivation . . . . . . . . . . . . . . 1

1.2 Outline and contributions . . . . . . . . . . . 2

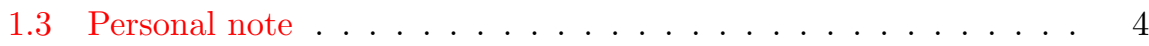

2 Setup and evaluation of a stiffness measurement technique $\quad 5$

2.1 Arm stiffness in biological motor control . . . . . . . . . . 5

2.1.1 Theoretical background . . . . . . . . . . . 5

2.1.2 Methods for stiffness estimation . . . . . . . . . . . 8

2.2 System setup . . . . . . . . . . . . . . . . 9

2.2.1 Experimental method . . . . . . . . . . . . . . 99

2.2 .2 Data processing . . . . . . . . . . . . . . . 15

2.2 .3 Stiffness estimation . . . . . . . . . . . . . 17

2.2.4 Stiffness visualisation and characteristics . . . . . . . . 18

2.3 Discussion of the reliability of the method . . . . . . . 20

2.3 .1 Pilot study . . . . . . . . . . . . . . . . 20

2.3 .2 Estimation of variability . . . . . . . . . . . . 22

3 Arm stiffness in three-dimensional space $\quad 25$

3.1 Participants . . . . . . . . . . . . . 25

3.2 Endeffector stiffness in three-dimensional space . . . . . . . . 25

3.2.1 Variation of hand stiffness with hand position . . . . . . 25

3.2.2 Symmetric versus antisymmetric stiffness component . . . 27

3.2.3 Hand stiffness in external force fields . . . . . . . . . . . 29 
3.2.4 Variation of hand stiffness with arm posture . . . . . . . 31

3.3 Discussion . . . . . . . . . . . . . . . . . 37

3.3.1 Technical and individual challenges . . . . . . . . . 37

3.3.2 Recording time as a practical limit . . . . . . . . . 37

3.3.3 Reproduction and extension of classical results . . . . . . 37

3.3.4 Distinguishability of positions . . . . . . . . . . . . . . 38

3.3.5 Summarising judgement of the method . . . . . . . . . . 38

4 An experimental framework for movement studies $\quad 39$

4.1 Introduction . . . . . . . . . . . . . . . 39

4.2 General methods . . . . . . . . . . . . . . . . . 40

4.2.1 Setup overview ................. . . 40

4.2.2 Details on virtual reality . . . . . . . . . . . . . . 42

4.2 .3 Simulator ..................... . . . 42

4.3 Specific protocol for subsequent studies . . . . . . . . . . . . 44

4.3.1 Framework of the "Probability experiment" . . . . . . . . 44

4.3.2 Specification of a trial ............... . . 44

4.3.3 Technical details .................. . . 45

4.3.4 Experimental procedure .............. . 46

4.4 Participants . . . . . . . . . . . . . . . . 47

$5 \quad$ Planning and replanning of fast movements $\quad 48$

5.1 Introduction . . . . . . . . . . . . . . . . . 48

5.2 Trajectory formation and motor primitives . . . . . . . . . . . 50

5.2 .1 Motor planning ................ 50

5.2 .2 Primitives ................... 50

5.2 .3 Error correction ................ . 50

5.2 .4 Autopilot ................... 51

5.3 Data processing and modelling . . . . . . . . . . . 51

5.3.1 Trajectory-based data processing . . . . . . . . . 51

5.3.2 Bell-shaped velocity profiles and replanning . . . . . . 53

5.4 Results of trajectory-based analysis . . . . . . . . . . . . 55

5.4.1 General results on task performance . . . . . . . . . . 55

5.4 Trajectory timing and replanning . . . . . . . . . . 58

5.4.3 Modelling of direct movements . . . . . . . . . . . . 63

5.4.4 Replanning in terms of bell-shaped velocity profiles . . . . 63

5.4.5 On the question of symmetry of replanning process . . . . 64 
5.5 Summary of trajectory-based analysis . . . . . . . . . 71

6 Motor decisions under time pressure $\quad 73$

6.1 Introduction . . . . . . . . . . . . . . . . 73

6.2 Motor decisions . . . . . . . . . . . . . . . . . . 75

6.2.1 Bayesian decision theory and accumulation of evidence . . 76

6.2.2 Handedness and decisions . . . . . . . . . . . . . 76

6.3 Data processing and modelling . . . . . . . . . . . . . 77

6.3.1 Event-based data processing . . . . . . . . . . . . . 77

6.3.2 Linear model of the decision process . . . . . . . . . 78

6.3.3 Nonlinear model of the directional choice . . . . . . . . 79

6.4 Results of event-based modelling . . . . . . . . . . . . . . . 81

6.4.1 Stereotypical decision patterns . . . . . . . . . . . . 81

6.4.2 Linear model of attention span . . . . . . . . . . . . . 87

6.4.3 Correctness vs. simplicity under time pressure . . . . . 90

6.4.4 "Internal" dynamics subserving directional choice . . . . . 93

6.5 Summary of event-based analysis . . . . . . . . . . . . . 102

$\begin{array}{lll}7 & \text { Overall summary } & 104\end{array}$

A Appendix 115

A.1 Supplementary material . . . . . . . . . . . . . . . . 115

A.1.1 Overview of behavioral regularities in subsequent trials . 115

A.1.2 Full compilation of sessionwise ARMA error plots . . . . . 115

A.1.3 Kink count tables . . . . . . . . . . . . . . . 115

A.1.4 Error correction in terms of bell-shaped velocity profiles . 115

A.2 Technical details of the SensAble Phantom 3.0 6DoF . . . . . . 134

A.3 Toolbox for stiffness data analysis . . . . . . . . . . . . . . . 134

A.4 Toolbox for timed via-point movements . . . . . . . . . . 135

A.4.1 Script language for specification of experiments . . . . . 135

A.4.2 Hierarchy of experimental types and their default settings 137

$\begin{array}{ll}\text { B List of own publications } & 140\end{array}$

$\begin{array}{ll}\text { Bibliography } & 140\end{array}$ 



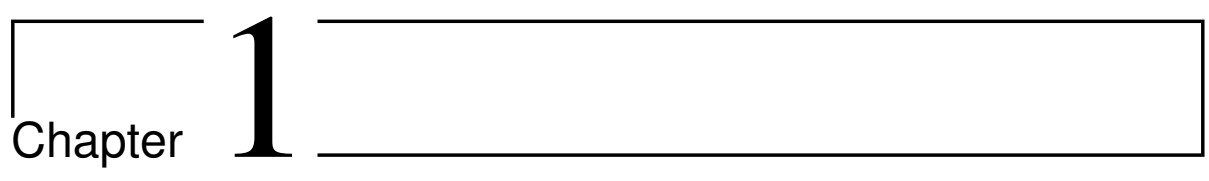

\section{Introduction}

\subsection{Motivation}

Humans constantly interact with their environment and produce a vast variety of arm postures and movements as well as fluent transitions between them in every day life. In these processes the interplay between the control strategy applied by the nervous system and the biomechanical apparatus of the body is highly optimised. This close interaction forms the motor system during the early developmental stages of the individual. It is a challenge in the field of motor control to reveal characteristic features of the system and to design experimental procedures to shed light on the underlying control mechanisms. This research not only targets at an understanding of our bodies, but also on new ideas for the field of prosthesis and robotics.

Arm movements are characterised by position, speed, accuracy and stability. The control of these important features also inspires the design of control strategies for robots. The stability of the limb against external disturbances is characterised by the so-called stiffness. If it is discussed in a Cartesian coordinate frame attached to the hand it is called endeffector stiffness. This key quantity in studies of arm posture summarises the spring-like behaviour of the limb, and serves as a measure of the resistance against external disturbances. Since it is not only influenced by passive material properties but also can be adapted actively (cf. Franklin et al., 2007; Gomi and Osu, 1998), robotisists became interested in it (see eg. Yang et al., 1993; Migliore et al., 2005; Diftler et al., 2011). 
This thesis studies two major aspects of motor control: posture and movement. In the first part, a setup for estimating human endeffector stiffness in three-dimensional space is developed. Classical approaches usually examined endeffector stiffness in the arm which is restricted to a horizontal plane in front of the shoulder and lead to two-dimensional estimates. The method, which I developed, overcomes this restriction and can be used to study stiffness patterns in the natural workspace of the human arm. Specifically the approach can be used to assess endeffector stiffness in three-dimensional hand space. In combination with additional tracking methods also joint stiffness calculation could be accomplished. Slight asymmetries in the estimated stiffness data are taken as a hint to the relation of control strategies to the existing hardware. In the second part, asymmetry in stereotypical arm movements is studied in right- and left-handed participants using both their preferred and non-preferred hand to carry out via-point movements to a final movement target that can be located either left or right behind an obstacle. Since the final target location was not revealed at the beginning of the movement but just at a critical time when the decision whether to pass the obstacle at the left or the right side already should be made, participants had to guess a direction based on previous experience or own assumptions about the setup. Our results indicate no effect of handedness in decisions on compositional arm movements. Furthermore, the participants showed a stronger bias towards movements to one or the other side for passing the obstacle, the faster their movements were. This velocity dependence of the stereotypicity of directional choices can be understood as a "heuristic" in human motor decision behaviour to simplify the task. A discussion of this idea in relation to cultural impriting is provided, and consequences for the design of robot control strategies to handle motor tasks under time pressure are drawn.

\subsection{Outline and contributions}

This section provides an overview of the main contributions of the presented thesis and lists relevant publications.

- In Chapter 2 I provide a detailed view of my developed approach of measuring arm stiffness in the redundant human $\operatorname{arm}^{1}$ in natural postures in everyday workspace. The technical part includes a toolbox which allows

\footnotetext{
1 "Redundant" refers to the larger number of degrees of freedom (four joint angles) that can be controlled by the central nervous system compared to the number of dimensions available at the endeffector. Given an endeffector position, joint angles are not uniquely defined.
} 
automated offline analysis of the measurements. Moreover, the performance of the approach is analysed.

Relevant publications: Fiedler (2007), Fiedler et al. (2008).

- Chapter 3 shows the results of applying the presented technique to estimate endeffector stiffness in several locations in workspace and in different external force fields. A number of classical arm stiffness results could be reconstructed in three-dimensional space. I confirmed the position- and posture-dependence of stiffness magnitude and orientation. However, I found that the direction of highest stiffness tends to be aligned approximately with the forearm axis, and not with the hand-shoulder axis as suggested in the literature.

Relevant publications: Fiedler and Herrmann (2009b).

- In Chapter 4 I present a general framework that can be used for designing experiments that target on motor planning, trajectory generation, and motor decision behaviour in humans. The framework provides means for a user-friendly arrangement of movement targets in a virtual reality setup as well as simplified hardware control of the utilised robot manipulator. In addition, I outline the specific application of this toolbox for the implementation of a two-alternative forced-choice motor task.

Relevant publications: Fiedler et al. (2010).

- Chapter 5 contains the results of the analysis of this motor task in the light of trajectory planning and replanning. Furthermore, a detailed analysis of movement timing and velocities during a critical phase of task execution is presented. Handedness as well as hand usage are examined in their influence on the replanning process. Also a discussion of the replanning process in cases of erroneous motor decisions and an analysis in terms of the superposition of movement primitives can be found in this chapter.

Relevant publications: Fiedler and Herrmann (2011).

- Chapter 6 highlights typical decision behaviour which is a stereotypical choice of mainly one movement direction. I relate this phenomenon to the strategy of passing the intermediate movement target and study possible influencing factors, such as own behavioural history and previously per- 
ceived visual stimuli. Finally, a nonlinear model of the underlying process is presented.

Relevant publications: Fiedler et al. (2010).

Detailed plots showing data from individual participants are placed in the Appendices and accompanied with a summarising figure in the section where they are discussed.

\subsection{Personal note}

This thesis has to be understood as a computer scientists excursion into the field of motor control

- to learn about the way of thinking in a field that deals with natural subjects instead of fully controllable ${ }^{2}$ automata, and

- to learn from nature for the design of more performant devices to achieve an increased acceptance by humans.

In the following different perspectives are adopted. Classical motor control approaches to the topics of human endeffector stiffness, and motor decisions and trajectory formation under time pressure are taken. Before discussing results within the respective contexts a number of technical questions, such as measurement refinements, are treated. Furthermore, from time to time I make some philosophical side notes about how specific results may serve as a source of inspiration for robot and prosthesis design.

2 "Controllable" not to be understood in a control-theoretical reading. 


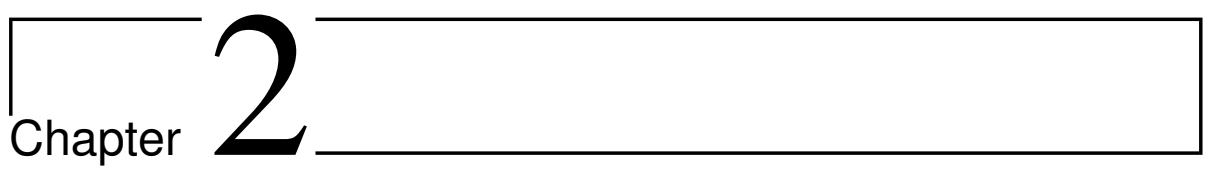

\section{Setup and evaluation of a stiffness} measurement technique

\subsection{Arm stiffness in biological motor control}

\subsubsection{Theoretical background}

Motor control deals with the information processing in preparation of and the generation of posture, movement, and skilled actions. Several theories have been suggested to unify the underlying processes. For example, posture as well as movement can be thought as being generated by the same sort of commands. The nervous system defines a sequence of muscle activations (cf. Bizzi et al., 1984), which are also called virtual trajectory (cf. Hogan, 1985). ${ }^{1}$ Feldman (1966) developed this so-called equilibrium point hypothesis. To understand its keystone one can consider the example of a single joint: an agonist and antagonist activation pattern is given as a descending signal (which means it is sent from the brain "downwards" to the body) to impose a flexion or extension at the joint. For each point in this sequence the muscles transform the activation into forces by contracting (according to their torque-length characteristics (cf. Mussa-Ivaldi et al., 1993)). These forces are balanced (or in equilibrium) in a certain posture. Figure 2.1 illustrates the force-length characteristics of agonist and antagonist (bold curves) as well as the resulting characteristics (thin line).

\footnotetext{
${ }^{1}$ Movement and even more so, posture, are defined by the activity in sensory motor loops, whereas here for the moment only the open-loop aspect is considered.
} 
The equilibrium point is characterised also by its dynamical properties. An external force that is exerted on the joint causes the control mechanism to react by a temporary deflection. Furthermore, the muscle activation results in a typical stiffness at the equilibrium point. It stabilises the endeffector against external disturbances. Given a fixed equilibrium point stiffness can be found as the slope of the resulting net characteristics.

Simply speaking, movement is generated by matching the virtual trajectory to the actual one. The quality of the match is evaluated from the interplay between forces arising from the movement and the ones resulting from arm stiffness.

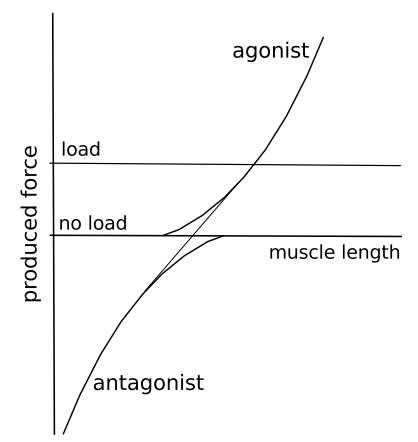

Figure 2.1: Illustration of muscular force-length characteristics at a single joint: A given activation results in a muscle length that produces a certain force. Stiffness is the linear approximation to the net result of all involved characteristics (cf. Feldman, 1966).

This interplay between controlled and intrinsic properties of a biomechanical system has found a lot of attention in the history of behavioural neuroscience. Most existing studies examined endeffector and joint stiffness in a plane. Usable degrees of freedom of the arm are reduced by fixation with cuffs or ropes from the ceiling to support the upper arm. Flash and Mussa-Ivaldi (1990) studied posture in a horizontal plane at shoulder height and showed that endeffector stiffness depends on hand position and varies throughout the workspace. They found direction of the highest stiffness aligned with the hand-shoulder axis and provided a discussion of the relation of this fact to joint stiffness.

Flash and Gurevich (1991) drew a line to levels of muscle activation and proposed a linear superposition model that explained the constant endeffector stiffness in cases of additional external loads in terms of a compensation for thereby additionally imposed forces at joint level. Tsuji et al. (1995) estimated not only endeffector stiffness but also contributions of inertia and viscosity during the displacement. 
Acosta et al. (2000) stated that static stiffness is influenced not only by length-tension characteristics ${ }^{2}$ but also by crossbridge elasticity and the tonic stretch reflex. In movement the situation becomes much more complex as interaction moments can no longer be ignored (see Tsuji et al., 1995).

According to Buneo et al. (1997) the brain must have an internal model of the arm to do posture-dependent transformation for the prediction of torque directions. These authors also found that humeral rotation does not influence torque direction, however, elevation and azimuth do matter along with the plane of arm and elbow angle.

Gomi and Kawato (1996) argue against the equilibrium point hypothesis stating that joint stiffness is too small to stem from the proposed simple mechanisms. Furthermore, they showed that the equilibrium point trajectories sometimes cannot be reasonably well computed from measured stiffness and torques.

Gomi and Osu (1998) studied the influence of cocontraction and force production on stiffness. They found that cocontraction changes shape and orientation. In force production joint stiffness is proportional to single-joint torque of the according joint, while the cross-joint stiffness is correlated to the elbow torque. Changes in joint stiffness are used to regulate endeffector stiffness for interaction with the environment.

Osu and Kawato (1999) studied cocontraction and force production by means of EMG and stiffness. Monoarticular muscles produce stiffness for posture, biarticular ones contribute to movement and force production.

A later study Franklin and Milner (2003) suggest a linear dependence of all stiffness entries on elbow and shoulder torque (which contradicts Gomi and Osu (1998)). Simulations showed that all joint stiffness coefficients need to vary with net torque to gain stability. On application of increased force, they found also increased stability - presumably caused by cocontraction.

Darainy et al. (2004) show that impedance control involves learning and adaption. They experimentally confirmed that there are at least two independent commands for cocontraction: one for the elbow and two-joint muscles; and one for the shoulder muscles. Forces along a single axis rotate the stiffness in the course of learning, isotropic forces give rise to a general increase.

Franklin et al. (2007) showed that for reaching movements in divergent force fields the major axis of stiffness rotates towards the instability (i.e. stiffness

\footnotetext{
${ }^{2}$ First studied by Rack and Westbury (1969) who found that in isometric conditions higher activations of the muscle results in a higher stiffness.
} 
increases in direction of force field). This is an additional proof that there is control of limb impedance and selective adaption to environment.

"The posture of the arm at a given hand location does depend on the starting location of the movement and that, consequently," (Soechting et al., 1995, p. 6271). Donders' law does not apply to arm movements. Purely kinematic modelling is not sufficient for the prediction of final posture. These results established the working hypothesis that final posture minimises the work that is needed to transport the arm there.

Sanger (2000) suggests principle component analysis as a method to study the combined properties of controller and musculoskeletal system.

By presenting the benefits of the consideration of interaction torques in movement data analysis Ketchama et al. (2004) put further emphasis on the importance of biomechanical aspects to movement generation.

Stiffness patterns of the human motor system and their stabilising effects inspired the field of robotics to apply stiffness control (which not only targets joint angles but also joint stiffnesses using a specific setup of servo motors) resulting in a desirable increase in movement stability even in the presence of unpredictable external disturbances (cf. eg. Yang et al., 1993; Migliore et al., 2005). This important step in robotics contributed to studies on human-robot interaction where the whole situation is too complex for analytical modelling which is typically applied to highly structured industrial setups.

\subsubsection{Methods for stiffness estimation}

Various research groups apply methods of stiffness measurement that use specifically designed robot manipulators. First single joint measurements (hold and release paradigm) in humans were carried out by Feldman (1966). Bizzi et al. (1984) used a single joint manipulator to impose angular displacements in a monkey's arm during movement. Interestingly, (assisting) displacements were rather large (up to 30 degrees in a movement of 60 degrees).

Mussa-Ivaldi et al. (1985) pioneered in measuring multi-joint stiffness using a custom-made two-link planar manipulator. They imposed PD-controlled steplike $\left(\right.$ small $^{3}$ ) hand displacements in eight directions (120 ms physiologically induced ramp, $1.5 \mathrm{~s}$ holding phase), and measured the restoring forces.

${ }^{3} 8-14 \mathrm{~mm}$. 
Tsuji et al. (1995) used a strong manipulator to impose PD-controlled displacements. They recorded the restoring forces, and estimated inertia, viscosity, and stiffness from the data.

Gomi and Kawato (1996) developed a parallel link drive air-magnet floating manipulandum which could exert higher forces. They studied hand movements of $40 \mathrm{~cm}$ amplitude (about 1s duration) along a reference trajectory. Small pushpull displacements in the range of $6-8 \mathrm{~mm}$ were imposed for about $200 \mathrm{~ms}$ before, during and after movement. Franklin et al. (2007) used a slightly different protocol: $100 \mathrm{~ms}$ push, $100 \mathrm{~ms}$ hold, $100 \mathrm{~ms}$ pull.

Xu and Hollerbach (1998) extended their existing 1D setup to work in 2D and worked on a parameter identification scheme for moving arms. They imposed a torque to the joints and tracked the angular displacement to characterise reflex activity in multi-joint systems. Furthermore, a voluntary contribution to rapid motor responses was revealed (see e.g. Pruszynski et al., 2008).

Acosta et al. (2000) developed a very strong manipulator to impose stochastic displacements of different frequencies during movement and record restoring forces. Peak-to-peak displacement was about $40 \mathrm{~mm}$. The plane where the measurement are carried out is rotatable. They used a parameter identification scheme originally published by Perreault et al. (1999).

\subsection{System setup}

\subsubsection{Experimental method}

\section{Fundamentals}

The idea of the procedures and methods here is similar to that from previous studies in the horizontal plane, yet there are two major differences:

- recordings were done in three-dimensional (hand) space with four degrees of freedom at the arm, thus in the situation of underdeterminance;

- for technical reasons this redundancy in the system did not allow a definition of displacements, but only the imposition of displacing forces. Hence, displacements were recorded.

Subjects maintained their posture against external disturbances. They were explicitly told to not voluntarily interfere with the effects the external forces might have on their hand. Instead, their attention was drawn to one of two 
distraction tasks: either counting from one to three during the displacement phase, or reading English words from a screen. Participants in the experiment with the second condition were kept completely naïve about the basic idea of the experiment. To strengthen the belief in the distraction task, they even were equipped with a microphone which was pretended to be tested before the experiments began.

\section{Procedure}

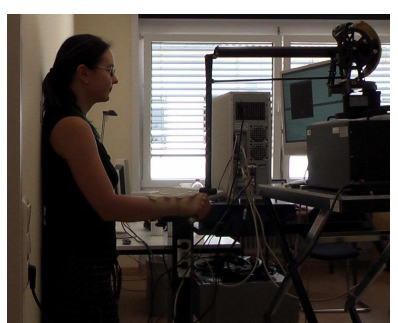

Figure 2.2: Subject standing in front of the manipulandum. The exact position is well-defined by markers on the walls and on the floor. Her back is supported by a wall to prevent swaying. She holds the handle of the manipulandum. The wrist is fixated. Visual feedback for hand positioning is provided on a computer screen.

Subjects stood in front of a six-joint manipulandum (see Appendix A.2 for technical details) with their back leaned to a wall to prevent swaying (cf. Figure 2.2). Standing posture was kept constant using markers on the floor and the wall, so that the participants could take a break, whenever they wished to, without imposing additional bias to the recorded data by choosing a different posture afterwards. The subjects grasped the handle of the machine which could move freely in three-dimensional space. Their wrists were restrained with a brace to prevent movements. Before starting the actual experiment the participants could get used to the frictionless movements with the handle in their hands. The manipulandum was used to track their hand position and the orientation of the handle, as well as to apply forces to the hand during the displacement phase of each trial. During positioning phase, visual feedback of the hand position as well as handle orientation was provided on a computer screen (in a block-world-like fashion using OpenGl). A red ball indicated the hand, an attached cone the handle of the machine (cf. Figure $2.3 \mathrm{~B}$ ).

Hand drift monitoring Before each recording session the steadiness of the subjects' hand was tested in a 60 seconds postural task. The subject had to bring the hand into a starting position guided by visual feedback and then keep it there for 60 seconds. During this time visual feedback was switched off. Afterwards, these data then were used to estimate a hand drift speed threshold 

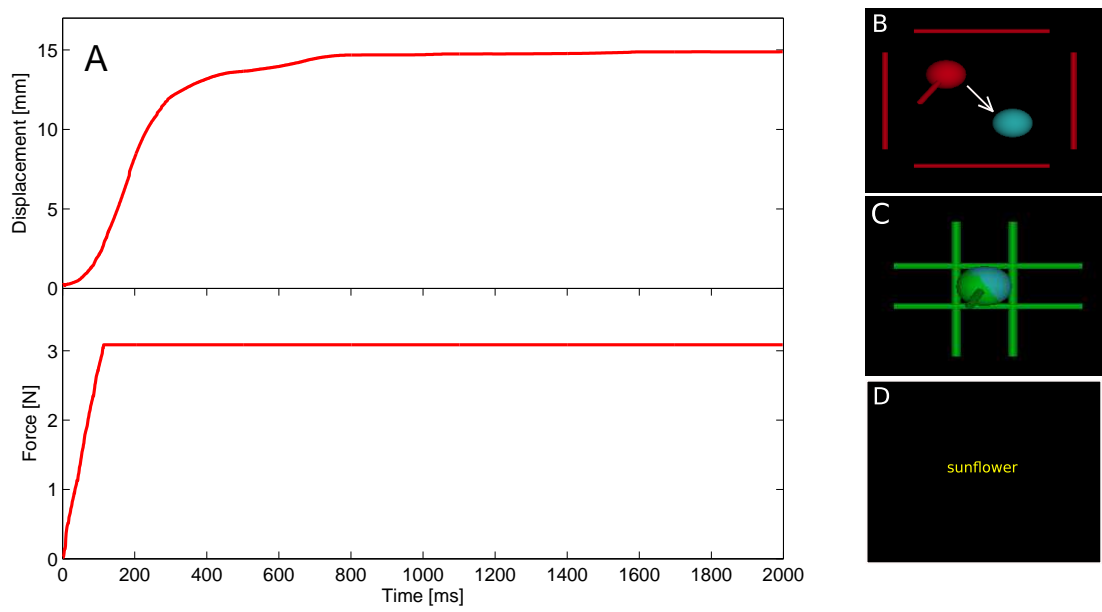

Figure 2.3: Schematic drawing of force and displacement traces (A) as well as provided visual feedback (B-D) of a typical session. A: Upper graph: Euclidean distance of a reference point at the subject's hand to the starting point of the experiment. Lower graph: Force strength applied by the machine to the subject's hand. After an initial positioning phase $(t<0)$ with zero force, a displacing force is ramped on $(0<t<250 \mathrm{~ms})$, resulting in a displacement of the hand. B: Visual feedback during positioning phase. The blue ball indicates the starting position. The red ball with red grid for improved depth perception represents the subject's hand. The red stick attached to the ball indicates the direction of the handle of the machine. C: On precise positioning of the hand at the starting point visual feedback is turned green. D: During the application of the force feedback of the hand and starting position is occluded. The computer screen shows a word which has to be read by the participant.

$v_{\text {thres }}$ which was used during data evaluation to define what had to be considered as the individual 'no hand movement'.

It was made sure that the subjects held the handle in a reproducible way by showing them to markers on the handle and instructing them to release it and grasp it again until they internalised a consistent grasp.

Definition of a trial A typical trial consisted of three phases (see Figure 2.3):

Starting phase: The participants had to bring their hand to a starting position displayed as a blue ball on the computer screen. Starting position was reached when being with the hand as close to the start position $\vec{x}_{s}$ as a predefined precision $d_{s}$ required. $d_{s}$ was chosen according to the subjects' steadiness of the hand and typically ranged between $0.5 \mathrm{~mm}$ and $1.5 \mathrm{~mm}$. On successful positioning the red feedback sphere for the hand turned green. This starting position had to be held for a time $t_{s}$ (typically around $600 \mathrm{~ms}$ ). The combination of 


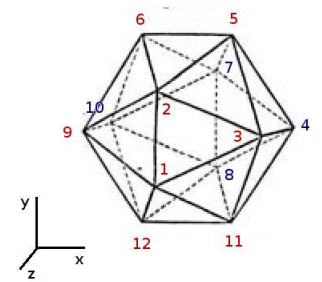

Figure 2.4: The twelve directions of the displacing forces are chosen centre-out in an icosahedron.
Table 2.1: Directions of the twelve displacing forces $(\mathrm{s}=0.618)$.

\begin{tabular}{|c|c|c|c|}
\hline 1 & 2 & 3 & 4 \\
\hline$(0,-\mathrm{s}, 1)$ & $(0, \mathrm{~s}, 1)$ & $(1,0, \mathrm{~s})$ & $(1,0,-\mathrm{s})$ \\
\hline 5 & 6 & 7 & 8 \\
\hline$(\mathrm{s}, 1,0)$ & $(-\mathrm{s}, 1,0)$ & $(0, \mathrm{~s},-1)$ & $(0,-\mathrm{s}, 1)$ \\
\hline 9 & 10 & 11 & 12 \\
\hline$(-1,0, \mathrm{~s})$ & $(-1,0,-\mathrm{s})$ & $(\mathrm{s},-1,0)$ & $(-\mathrm{s},-1,0)$ \\
\hline
\end{tabular}

$d_{s}$ and $t_{s}$ was chosen based on the individual hand drift speed threshold $v_{\text {thres }}$. After an additional random delay between 100 and $300 \mathrm{~ms}$ the displacement phase began ${ }^{4}$.

Displacement phase: Visual feedback of hand and starting position was switched off. Instead, the screen was kept black, if the participant had to do the counting task, or showed a randomly chosen English word ${ }^{5}$ which had to be read out loudly in the reading distraction task. Simultaneously, a displacing force was ramped on. Force direction was randomly chosen centre-out of a set of twelve directions uniformly distributed on a sphere (cf. Figure 2.4 and Table 2.1) Force strength was individually chosen based on a set of test trials such that the resulting displacement was small in the sense of stiffness measuring paradigms (cf. Mussa-Ivaldi et al. (1985)).

Movement phase: After counting or after the word was read from the screen the participants had to move their hand to the opposite direction as where the force came from. This was done to indicate a clear end of the trial. Then (after 2.5 to $3 \mathrm{~s}$ ) the displacing force was switched off again.

Sessions Each session with a new subject began with three to five test repetitions of 36 displacements. The strength of the displacing force first was chosen based on a gender and sports training state based heuristics, and then adjusted in course of the testing time to cause small displacements. The test recordings furthermore allowed the participant to get used to the setup.

\footnotetext{
${ }^{4}$ This random delay in combination with the distraction task prevented the participants from anticipatory changes in arm stiffness (cf. Biryukova et al., 1999).

${ }^{5}$ Ispell dictionary, version 3.2 .06 on Suse 9.0
} 
Afterwards the experiment began according to one of the protocols. Usually data for one protocol with different setup parameters were recorded in one session.

Each experimental session was followed by another drift monitoring minute which was used to judge the fatigue caused by the session.

Furthermore, forearm and upper arm length were determined after visually estimating the rotation axes of the joints.

\section{Available protocols}

Three main protocols which target on the role of the most relevant influencing variables - force, position and, posture - were implemented.

In the position variation protocol, the starting position of the hand was given. Since the subject's arm comprises four degrees of freedom, one was not determined by the task. For a given hand position this means that the rotation angle of the elbow around the hand-shoulder axis could be chosen at comfort. Recordings were done in various hand positions all over the workspace

In the posture variation protocol all degrees of freedom of the arm were predefined. This protocol involved an initial test of the working range of the participants' arm for a given hand position $\vec{x}_{s}$ : First the subject had to bring the hand to the starting position ten times. The average elbow rotation around the hand-shoulder axis $\vec{o}_{c}$ ( $c$ denotes comfort) was approximated by the mean angle between the handle of the manipulandum and the z-axis of the external workspace. Since only one additional degree of freedom had to be assessed this measure was sufficient to uniquely define the elbow rotation with regard to the natural limits of the joints (cf. Winter (2005)). Afterwards the subject was told to rotate the elbow as far as possible anti-clockwise around the hand-shoulder axis while still keeping the hand in starting position $\vec{x}_{s}$. After ten of these calibration rotations the maximal rotation limit $\vec{o}_{m}$ ( $m$ denotes maximum) was calculated as the average over all maximally met rotations.

These average handle orientations $\vec{o}_{c}$ and $\vec{o}_{m}$ were used to define the handle orientations for the experiments:

- $\vec{o}_{1}=\vec{o}_{c}$

- $\vec{o}_{2}=\vec{o}_{c}+\frac{1}{3} \vec{o}_{m}$

- $\vec{o}_{3}=\vec{o}_{c}+\frac{2}{3} \vec{o}_{m}$ 
A trial was started if the direction of the handle matched the predefined orientation better than a given threshold. For current pointing direction $\vec{h}$ of the handle and given orientation $\vec{o}$ the criterion

$$
|\vec{h}-\vec{o}<0.1|
$$

was applied. It was intended to measure stiffness for the arm in these three postures (comfortable, elbow lifted by one third of the maximum elevation, and elbow lifted by two thirds of the maximum elevation) for several hand position. Due to the demanding procedure of repeatedly bringing the arm into the starting postures, leading to early abortions of the sessions or very high variance in the data this protocol was mainly expected to work in athletic participants. This could successfully been shown in one participant with a strong sports background.

The external force field protocol is a version of the position variation protocol with a constant force acting against the subject's hand all over the experiment. The strength of the external force field and the starting position were varied.

\section{Remark on the underlying assumptions}

There are a number of parameters influencing the quality of the recorded data. Here is a list of the assumptions underlying our approach .

1. Grasping of the handle is assumed to be perfectly stable and reproducible after releasing the handle. We have reduced the effects of grasp variations by restraining the wrist, by definition of the grasping position on the gimbal, as well as by averaging of several grasps.

2. Wrist restriction is thought to be optimal and should lead to only $4 \mathrm{DoF}$ at the arm. Here the necessary compromise between the discomfort induced by the restraining bandage and the remaining movability of the wrist has been shifted as much as possible towards immobilisation of the wrist joint.

3. Errors in anthropomorphic measurements are assumed not to affect the qualitative results. Measurements were repeated under different conditions and averaged. Compared to the more critical structural assumptions e.g. of mono-centric joints, the numerical errors can be neglected.

4. Estimation of joint angles from snapshot data is assumed to be correct (at least in vertical plane). 
5. Force displacements are sufficiently small so that they do not alter the equilibrium position.

While the caveats 3 and 4 would only matter in case of joint stiffness estimation, which turned out to be not feasible, and caveat 5 could be prevented by means of accommodation trials where force strength was adjusted, caveat 1 and 2 remained present throughout the experiments. Therefore, according variations were suppressed as much as possible.

\subsubsection{Data processing}

\section{Displacement extraction}
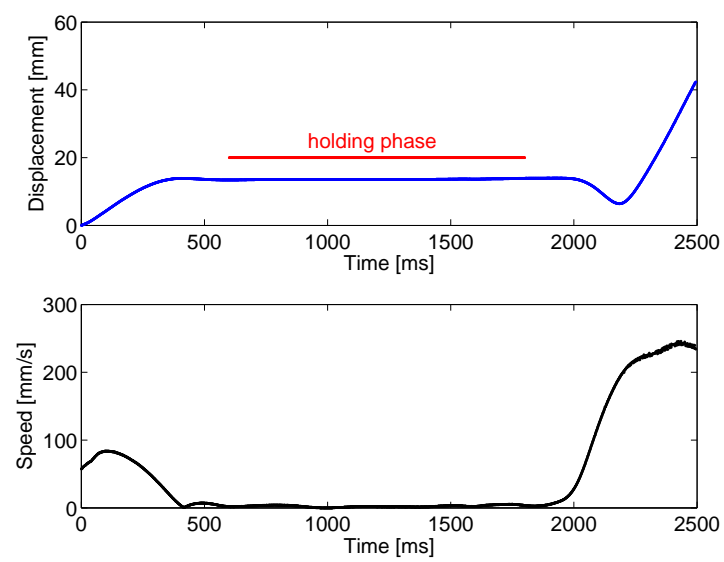

Figure 2.5: Illustration of data extraction during holding phase: Upper plot: Time course of the distance of the hand from the starting position for an example trial. Holding phase - where measurement of the displacement takes place - is marked with a red bar. Lower plot: Speed trace which is used to make the judgement about the holding phase.

Figure 2.5 shows an example of the time course of the hand displacement. The plateau between 600 and $1200 \mathrm{~ms}$ is called holding phase. The extent of the displacement is measured during this interval. Since the hand position is not completely steady throughout this holding phase a heuristic has been developed to decide what still can be considered as holding and what has to be rejected as spurious movements. For this purpose the hand position data $\vec{x}(t)$ is smoothed (using sliding mean with a window size of 10 data points), the velocity $\vec{v}(t)$ is computed using numerical differentiation and projected to the force direction $\vec{F}$. If this resulting velocity $\vec{v}_{\vec{F}}(t)$ is smaller than the drift speed threshold $v_{\text {thres}}$, 
the hand is considered as still. The drift speed threshold $v_{\text {thres }}$ is computed based on $60 \mathrm{~s}$ recording of keeping the starting position before the measurement session. For each instant of time the hand speed is computed and binned into a speed histogram (see Figure 2.6). $v_{\text {thres }}$ is chosen so that 98 percent of this speed data is below it. Typical values are around $3.0 \mathrm{~mm} / \mathrm{s}$ which resemble typical definitions of 'hand in rest' from previous studies (eg. McIntyre et al. (1996)).

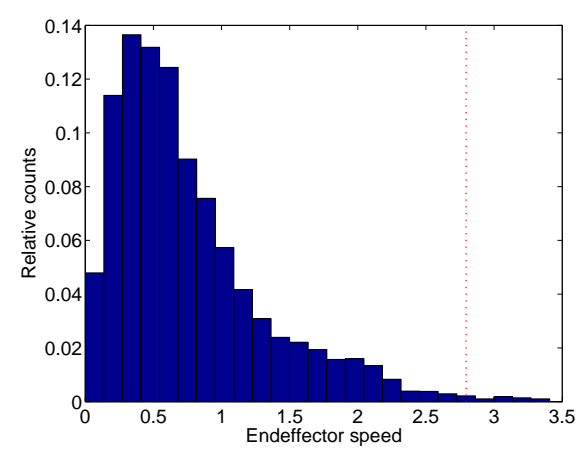

Figure 2.6: Endeffector speed histogram of 30 seconds of posture maintenance in the starting position without external disturbances. Individual drift speed threshold is set such that it is higher than 98 percent of all speed counts. Red dotted line indicates $v_{\text {thres }}$ at $2.79 \mathrm{~mm} / \mathrm{s}$.
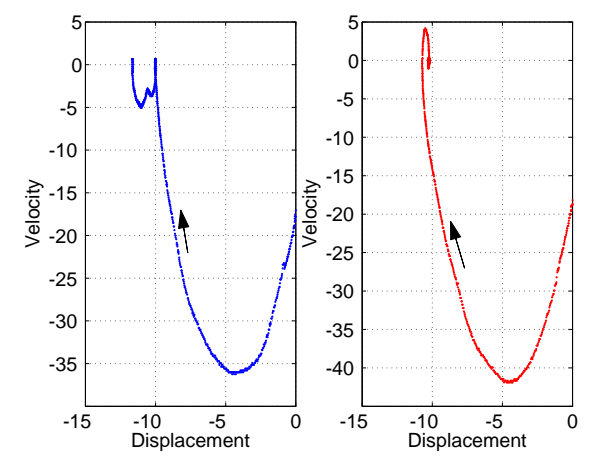

Figure 2.7: Illustration of "corrections", i.e. major changes in endeffector position that do not stem from an undisturbed decay of an oscillation around the equilibrium point. Left: Speeddisplacement diagram of a trial with a correction of about $2 \mathrm{~mm}$ in the end. Right: Speed-displacement of a trial without correction. Black arrows indicate the movement.

The displacement in each spatial direction was extracted for the complete holding phase for final computation of the mean displacement of that trial.

This automated processing was supported by manual check with a visual detection of the holding phase. The very few spurious trials were sorted out this way.

Analysis of voluntary corrections Since voluntary corrections ${ }^{6}$ by the participant - i.e. moving the arm back towards the 'felt approximation' of the starting point - would severely corrupt the results, a check for corrections is applied.

\footnotetext{
${ }^{6}$ I applied Feldman's definition of corrections (for details see Mihaltchev et al., 2005; Foisy and Feldman, 2006).
} 
For this purpose phase plots of single trials were analysed. Figure 2.7 shows the phase plots of two trials, one without correction where the trajectory comes to rest within the first two zero crossings of velocity, and one with correction where the final holding point moves further away from where it was originally directed to. It has to be assumed that in these trials the participants made voluntary corrections.

To compensate for this shift in the equilibrium position the displacement value from the time of the correction onset was taken. This results in a displacement error in submillimeter range, compared to one up to $5 \mathrm{~mm}^{7}$ when accepting the corrupted final displacement.

\subsubsection{Stiffness estimation}

\section{Endeffector stiffness estimation in posture}

Assuming a rigid-body model of the arm movement in joint space (as done in Biess et al. (2007); also cf. Tee et al. (2004)) with three degrees of freedom at the shoulder $(\eta, \theta, \xi)$ and one at the elbow $(\phi)$ summarised in the joint posture $\vec{q}=(\eta, \theta, \xi, \phi)$ the arm dynamics can be written as

$$
M(\vec{q}) \ddot{\vec{q}}+C(\dot{\vec{q}}, \vec{q}) \dot{\vec{q}}+N(\vec{q})=\overrightarrow{\tau_{d}}
$$

$M$ denotes the inertia matrix, $C$ the Coriolis matrix (including centrifugal force components), and $N$ the gravitational torques. In the interaction with an external force $F_{\text {ext }}$ this torque has to be produced additionally to move the arm. Hence, the resulting joint torque has to be

$$
\vec{\tau}=-J(\vec{q})^{T} F_{\mathrm{ext}}+\overrightarrow{\tau_{d}}
$$

with $J$ being the Jacobian of the transformation from hand to joint space.

Joint stiffness $R$ reflects the resistance of the arm (posture) against external (torque) displacements, hence

$$
R=\left(\frac{\delta \tau_{i}}{\delta q_{j}}\right)_{i, j=1 . .4}
$$

\footnotetext{
${ }^{7}$ This value is the maximum over all participants. Average correction size is between 2 and $3 \mathrm{~mm}$.
} 
This can be rewritten as

$$
R=J^{T}\left(\frac{\delta F_{i}}{\delta x_{j}}\right)_{i, k=1 . .4}\left(\frac{\delta x_{k}}{\delta q_{j}}\right)_{k, j=1 . .4} J+\frac{d J^{T}}{d \vec{q}} \vec{F} .
$$

Since endeffector stiffness is estimated in the static condition, only

$$
K=\left(\frac{\delta F_{i}}{\delta x_{j}}\right)_{i, k=1 . .4}
$$

remains after the switch to hand space.

Stiffness estimation Equation 2.6 was implemented using least square optimisation for applied forces $d \vec{F}$ resulting in an endeffector displacement $d \vec{x}$. Since the displacement is not too large, the equilibrium is kept.

Visualisation of endeffector stiffness For visualisation purposes the not necessarily symmetric stiffness matrix $K$ is decomposed into a antisymmetric $K_{a}$ and a symmetric part $K_{s}$ according to

$$
K_{s}=\left[\begin{array}{ccc}
K_{x x} & 0.5\left(K_{x y}+K_{y x}\right) & 0.5\left(K_{x z}+K_{z x}\right) \\
0.5\left(K_{x y}+K_{x y}\right) & K_{y y} & 0.5\left(K_{y z}+K_{z y}\right) \\
0.5\left(K_{x z}+K_{z x}\right) & 0.5\left(K_{y z}+K_{z y}\right) & K_{z z}
\end{array}\right]
$$

and $K=K_{a}+K_{s}$ (cf. Mussa-Ivaldi et al. (1985)). While the former represents the rotational part of stiffness, the latter contains all conservative components. The symmetric part of endeffector stiffness now can be visualised by interpreting $K_{s}$ as a transformation which acts on a sphere of unit displacements, resulting in a distribution of restoring forces that has the shape of an ellipsoid (which is the generalised version of the stiffness ellipse in Flash and Mussa-Ivaldi (1990)). A longer extension of the ellipsoid indicates a high stiffness in this direction.

\subsubsection{Stiffness visualisation and characteristics}

\section{Visualisation}

Endeffector stiffness is depicted as an ellipsoid that graphically shows the resulting restoring forces to unit displacements in the according directions. This a generalisation of the stiffness ellipse suggested by Flash and Mussa-Ivaldi (1990). The major axis of the ellipsoid indicates the direction of highest stiffness, i.e. 
the direction where the least displacement can be imposed using a given force strength. The minor axes point in directions of lower stiffness (allowing larger displacements).

In all pictures an additional colormap (ranging from blue to red for values $\left.\left[c_{\min }, c_{\max }\right]\right)$ is used to give a better impression of the third dimension that is missing in the plots. The upper value of the colour range provides a coarse estimate of the largest stiffness involved. Furthermore, images always show two views of the ellipsoid, one from the right side of the subject and one looking towards the computer screen from the participant's eye plane.

\section{Orientation, size and shape of the stiffness ellipsoid}

For a comparison of different stiffnesses $K_{1}$ and $K_{2}$ a set of measures was generated, using three-dimensional generalisations of the visually inspired ideas presented by Flash and Mussa-Ivaldi (1990). Let $K_{1}$ be a symmetric stiffness

matrix with eigenvectors $\vec{\lambda}_{1}, \vec{\lambda}_{2}$, and $\lambda_{3}$ (with $\vec{\lambda}_{1} \leq \vec{\lambda}_{2} \leq \vec{\lambda}_{3}$ ), and according eigenvalues $e_{1}, e_{2}$, and $e_{3}$. Furthermore, let $K_{2}$ be also symmetric with eigenvectors $\vec{\mu}_{1}, \vec{\mu}_{2}$, and $\vec{\mu}_{3}$ (with $\vec{\mu}_{1} \leq \vec{\mu}_{2} \leq \vec{\mu}_{3}$ ), and according eigenvalues $f_{1}, f_{2}$, and $f_{3}$. The size of the stiffness ellipsoid can be associated to its volume

$$
V=\frac{4}{3} \pi e_{1} e_{2} e_{3}
$$

The shape can be read from the ratios of the eigenvalues

$$
r_{1}=\frac{e_{1}}{e_{2}} \text { and } r_{2}=\frac{e_{2}}{e_{3}}
$$

For the relative orientation of the ellipsoid of $K_{2}$ to the one of $K_{1}$ the angle $\phi$ between their major axes $\vec{\lambda}_{1}$ and $\vec{\mu}_{1}$ has to be considered, as well as the angle $\xi$ between the orthogonal projection of $\vec{\mu}_{3}$ into the $\vec{\lambda}_{2}-\vec{\lambda}_{3}$ plane

$$
\vec{\mu}_{3}^{\prime}=\vec{\mu}_{3}-\frac{\vec{\mu}_{3} * \vec{\lambda}_{1}}{\left|\vec{\lambda}_{1}\right|^{2}} \vec{\lambda}_{1}
$$

and $\vec{\lambda}_{3}$.

When only considering one ellipsoid the relative orientation to a fixed coordinate frame can be taken (with $\left.\vec{\mu}_{1}=(0,0,1)^{T}, \vec{\mu}_{2}=(0,1,0)^{T}, \vec{\mu}_{3}=(1,0,0)^{T}\right)$.

In the following, $\phi$ is referred to as tilt, and $\xi$ as twist with respect to a given frame of reference. 


\section{Discussion of stiffness orientation}

According to a derivation by Flash and Mussa-Ivaldi (1990), in the nonredundant two-dimensional case the orientation of endeffector stiffness $K$ can be discussed in terms of single- and two-joint muscle contribution to the according joint stiffness $R=J^{T} K J$ with $J$ being the Jacobi matrix of the transformation from joint to hand space. The decomposition of joint stiffness into contributions from single-joint muscles at the shoulder $R_{s}$, and at the elbow $R_{e}$, and two-joint muscles $R_{t}$ leads to

$$
R=\left[\begin{array}{ll}
R_{s}+R_{t} & R_{t} \\
R_{t} & R_{t}+R_{e}
\end{array}\right] .
$$

For $R_{t}=R_{e}=0$ and $R_{s} \neq 0$ the major axis of $K$ points along the forearm (with an according eigenvalue of $R_{s} /\left(l_{f}^{2} \sin ^{2} \theta_{e}\right)$ ). In the contrary case of $R_{t}=$ $R_{s}=0$ and $R_{e} \neq 0$ the major axis of $K$ points along the radial axis of a polar coordinate system attached to the shoulder (with the eigenvalues coinciding at $r^{2} R_{e} /\left(l_{u}^{2} l_{f}^{2} \sin ^{2} \theta_{e}\right)$ ), with $r$ being the distance between hand and shoulder). An alignment of the major axis of $K$ with the upper arm axis can be found for $R_{e}=R_{s}=0$ and $R_{t} \neq 0$ (non-zero eigenvalue $R_{t} /\left(l_{f}^{2} \sin ^{2} \theta_{e}\right)$ ).

\subsection{Discussion of the reliability of the method}

\subsubsection{Pilot study}

Before collecting data for stiffness estimation, important parameters of the experiment were tested in their effects. These parameters are: positioning precision (maximal distance from starting point to still consider it as reached), positioning time (minimal time of correct positioning in the starting point in the above mentioned sense), ramping time (time till the displacing force is switched on completely), force strength (strength of the displacing force), and duration of one trial.

\section{Subjects}

In a pilot study the setup was tested in three right-handed subjects (2 male, 1 female), aged between 26 and 28. Their vision was normal or corrected to normal. None of the participants reported any neuromotor arm dysfunction. 


\section{Hand drift with and without support from the back}

For an evaluation of hand drift speed (in order to judge hand 'steadiness' later on) the subjects were asked to keep their hand in a predefined (central) position for 60 seconds. Hand position was tracked throughout this time. Visual feedback of the endeffector position on the screen was occluded. Figure 2.8 shows a comparison of two cases: (A) the participant standing freely in front of the manipulandum, (B) the participant is allowed to lean to a wall. From endeffector tracking (left subfigure) it can be seen clearly that the support by a wall reduces the drift in endeffector position. Moreover, breathing artifacts (oscillation of about $1 \mathrm{~mm}$ amplitude) can be seen in $\mathrm{x}$-direction (along the participants forearm) due to mechanical coupling ${ }^{8}$. Furthermore, leaning to the wall reduces the velocity spectrum (see right subfigure) by excluding sway components, resulting in a more frequent occurrence of lower drift speeds. So the backrest is an essential constituent of the setup.
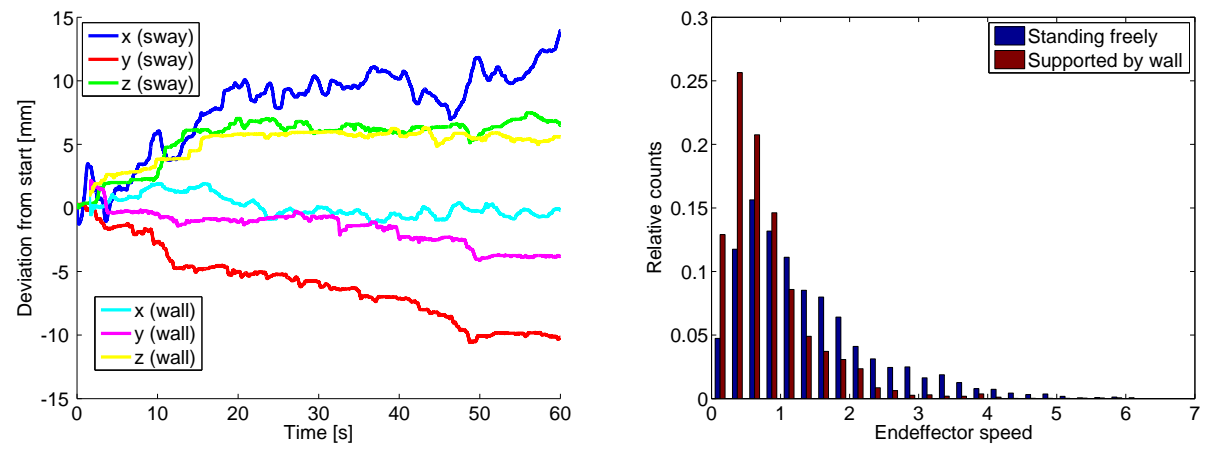

Figure 2.8: Comparison of endeffector drift characteristics of one participant standing freely and supported by a wall. Left: Coordinate deviation from starting point over time. Right: Speed histogram of both cases.

Calculation of the expected drift The distribution of possible deviations in all three directions of space for a given time interval $\delta t$ is calculated using $\vec{x}(t+\delta t)-\vec{x}(t)$. Table 2.2 shows a comparison of expected deviations for freely standing participants and the ones supported by a wall.

In a second set of tests different force fields were applied during the whole test to evaluate their influences on hand steadiness. On average they slightly

\footnotetext{
${ }^{8}$ Breathing effects during holding phase are negligible in the sense that for a breathing rate of about 14 times in a minute one breath takes more than four seconds compared to holding phases of only up to one second.
} 
Table 2.2: Expected endeffector deviations in $\mathrm{mm}$ within given time windows

\begin{tabular}{|c|c|c|c|c|c|c|}
\hline time window & \multicolumn{3}{|c|}{$0.5 \mathrm{~s}$} & \multicolumn{3}{c|}{$1.0 \mathrm{~s}$} \\
\hline direction of deviation & $\mathrm{x}$ & $\mathrm{y}$ & $\mathrm{z}$ & $\mathrm{x}$ & $\mathrm{y}$ & $\mathrm{z}$ \\
\hline standing free & 0.48 & 0.17 & 0.16 & 0.82 & 0.27 & 0.28 \\
\hline supported by wall & 0.18 & 0.13 & 0.12 & 0.32 & 0.22 & 0.20 \\
\hline
\end{tabular}

reduced endpoint variability in terms of a narrower speed histogram. So the protocol using external force fields did not suffer from an increase in additional noise.

\subsubsection{Estimation of variability}

Every measurement is influenced by a certain amount of noise resulting from various sources. Due to the rather small amount of data the measured variability may not completely resemble the true span of natural variability in the system. This led to the conclusion that the mean of the data cannot be recklessly taken as a solid basis for stiffness estimation. Therefore, a more elaborate statistical technique was considered.

The algorithm that is presented in the following provides not only an estimate for endeffector stiffness and the according variability based on a sample of recordings but also results in a suggestion of an optimal number of trials for each participant.

\section{$N$-fold cross-validation}

The procedure introduced in this section is also referred to as "leaving $n$ data points out" (or short: $\mathrm{LnO})^{9}$. Consider a set of $N$ measurements $\left\{\left(F_{i}, d x_{i}\right)\right\}$ (for simplicity just 1D here) with force $F_{i}$ and displacement $d x_{i}$. From this data set now $M$ samples are drawn without repetition to form an ensemble $E_{M}$. Now, stiffness $K_{M, 1}$ is calculated from it by minimising the cost function

$$
C=\sum_{j \in \operatorname{ind}\left(E_{M}\right)}\left|F_{j}-K_{M, 1} d x_{j}\right|
$$

(least square fit). To judge the variance this procedure is repeated $P$ times to produce the estimates $K_{M, 2}$ to $K_{M, P}$. The variance depicted in dependence on

\footnotetext{
${ }^{9} \mathrm{LnO}$ is a bootstrapping method (cf. eg. Freedman and Peters, 1984).
} 
the number of data points left out for the estimation results in a distribution that suggests two regimes: one where many data points are used and the variance is small, and one where only few data points are used and the according variance is high (cf. Figure 2.9 for a 1D example). The intersection of the two lines produced by a bilinear fit to the data gives an estimate of the optimal number of data points needed for a reliable estimation.

Table 2.3 shows the estimated stiffness along with the variance and the optimal number of data points needed for a reliable estimation. Although the optimum depends on the individual runs of the LnO algorithm for each number of repetitions a consistent suggestion for the variance and the number of data points is given. For all experimental data the $\mathrm{LnO}$ has been run with 50 repetitions.

Table 2.3: Dependence of estimated stiffness on number of repetitions in LnO algorithm

\begin{tabular}{|c|c|c|}
\hline $\begin{array}{c}\text { Number of } \\
\text { repetitions }\end{array}$ & $\begin{array}{c}\text { Estimated stiffness } \\
+/- \text { variance }\end{array}$ & $\begin{array}{c}\text { Optimal number of } \\
\text { left-out data points }\end{array}$ \\
\hline 10 & $0.488+/-0.024$ & 37 \\
20 & $0.523+/-0.023$ & 36 \\
30 & $0.505+/-0.024$ & 39 \\
40 & $0.503+/-0.028$ & 38 \\
50 & $0.475+/-0.023$ & 37 \\
60 & $0.506+/-0.027$ & 37 \\
70 & $0.491+/-0.025$ & 37 \\
80 & $0.514+/-0.025$ & 37 \\
90 & $0.499+/-0.027$ & 38 \\
100 & $0.506+/-0.025$ & 35 \\
110 & $0.484+/-0.028$ & 37 \\
\hline
\end{tabular}




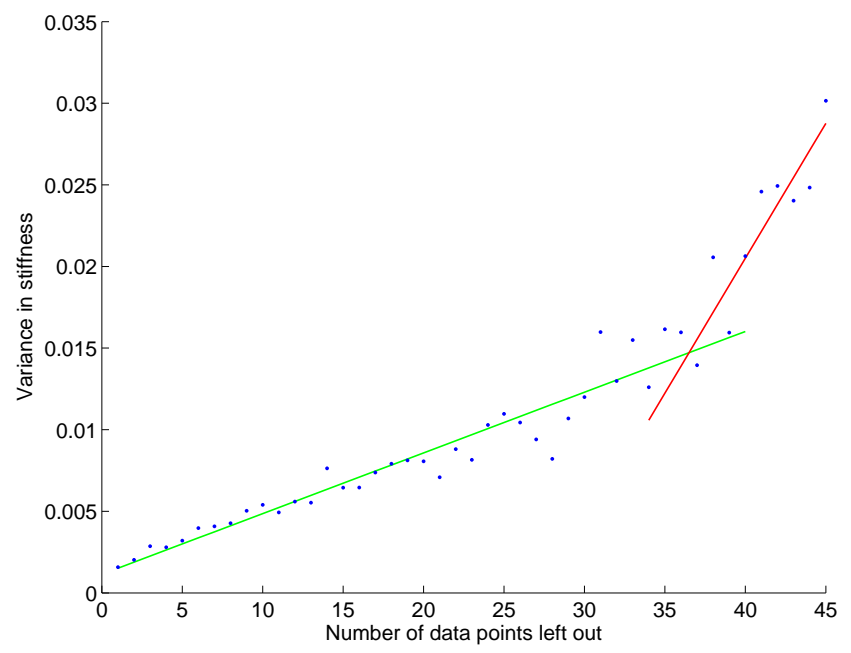

Figure 2.9: Illustration of LnO algorithm. Standard deviation in 1D stiffness estimate in dependence on the number of left-out data points. After creation of virtual data ensembles from 50 noisy 'measurements' of the system (noise uniformly distributed, fraction 0.4 of correct position). By randomly leaving out $\mathrm{n}$ data points and calculating stiffness and standard deviation from them, a bi-linear fit to standard deviation is used to find the optimal trade-off between minimal number of measurements and low variability (found at intersection of linear approximations). This leads to an estimation of the variance as well as to the necessary number of trials. Green and red line indicate best fitting lines to data (blue dots). Green: many data points used, thus, low variance (but big recording effort), red: few data points used, hence the outliers and noise dominate. 


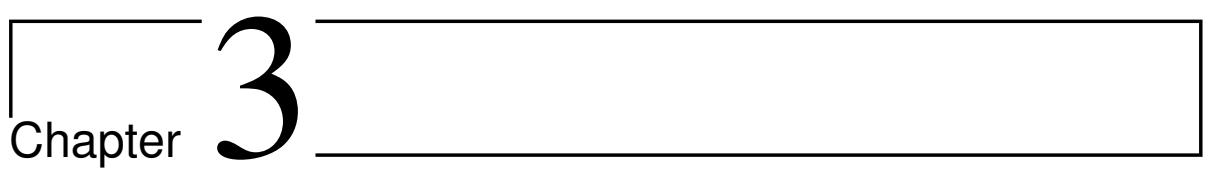

\section{Arm stiffness in three-dimensional}

\section{space}

\subsection{Participants}

18 right-handed subjects (aged 20 to 43) participated in this series of experiments. Ten of them completed the position protocol, eight the force field, and one in addition the posture protocol. As in the pilot study, their vision was normal or corrected to normal, and none of the participants reported any neuromotor arm dysfunction.

\subsection{Endeffector stiffness in three-dimensional space}

\subsubsection{Variation of hand stiffness with hand position}

\section{Stiffness in the sagittal plane}

Force-induced displacements without additional load were carried out in twelve healthy subjects in several locations of the daily used workspace (6 repetitions for each force direction $)^{1}$. In the 'long line' protocol seven positions $(10 \mathrm{~cm}$ apart each) were recorded. These data are available from two subjects. A 'short line'

\footnotetext{
${ }^{1}$ Consistency in the choice of the non-predefined degree of freedom (rotation of the elbow around hand-shoulder axis) was found across participants, in accordance with Tillery et al. (1995).
} 
of only four positions (with again $10 \mathrm{~cm}$ distance between neighbouring hand locations) was recorded in six participants. Data from further four subjects covers recording positions off from the line in front of the right shoulder.

Figures 3.1 show the position dependence of endeffector stiffness in the 'long line' condition. It can be seen that the major axis of hand stiffness tends to be aligned with the forearm axis. According to Section 2.2.4 this suggests that a significant degree of stiffness is produced at the shoulder while keeping the elbow (as well as the shoulder-elbow interaction component of stiffness) in a reactive range with a rather low stiffness.
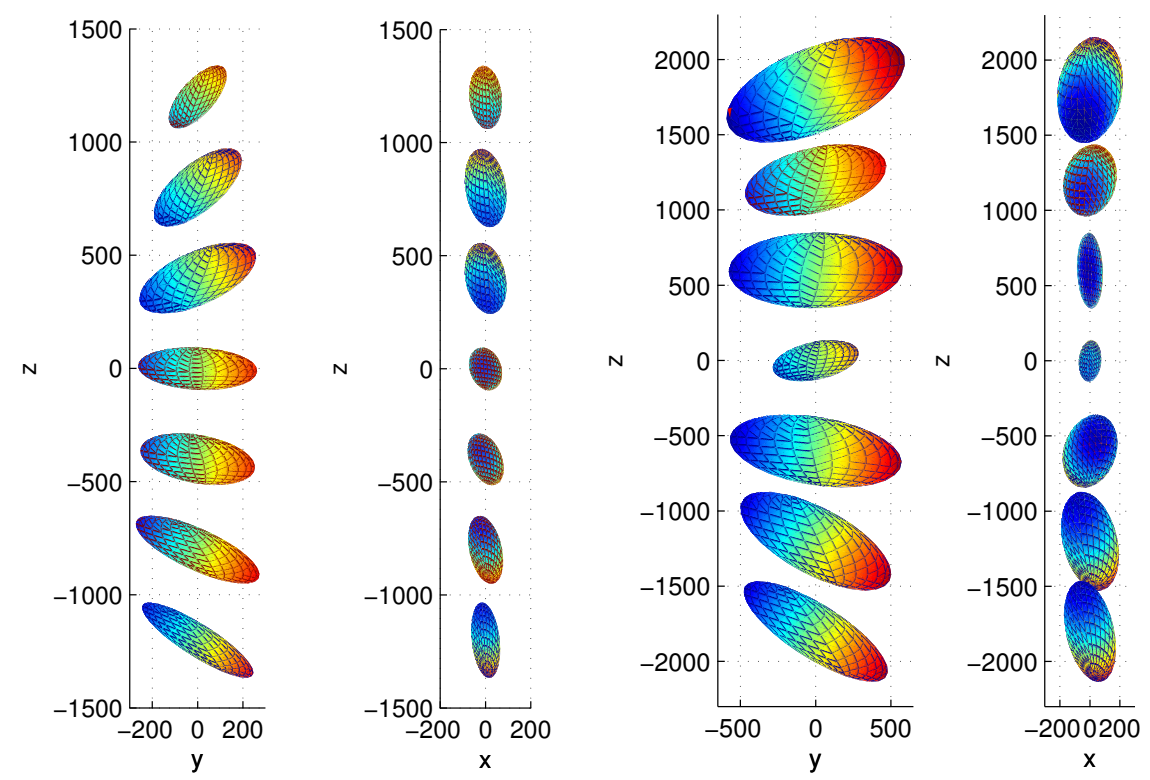

Figure 3.1: Endeffector stiffness along a vertical line in front of the participant's shoulder. Studied hand positions were $10 \mathrm{~cm}$ apart, three above and three below the central position (which is 90 degrees at the elbow, and zero at the shoulder). Left: Data from female participants. Right: Data from male.

An analysis that only considers the orientation of the endeffector stiffness is shown in Figure 3.2. The angle between x-axis and major axis of endeffector stiffness remains almost unchanged throughout recording positions. This is caused by the standard way of grasping the handle and afterwards bringing it to starting position (which causes a rightwards rotation of about 90 degrees). 

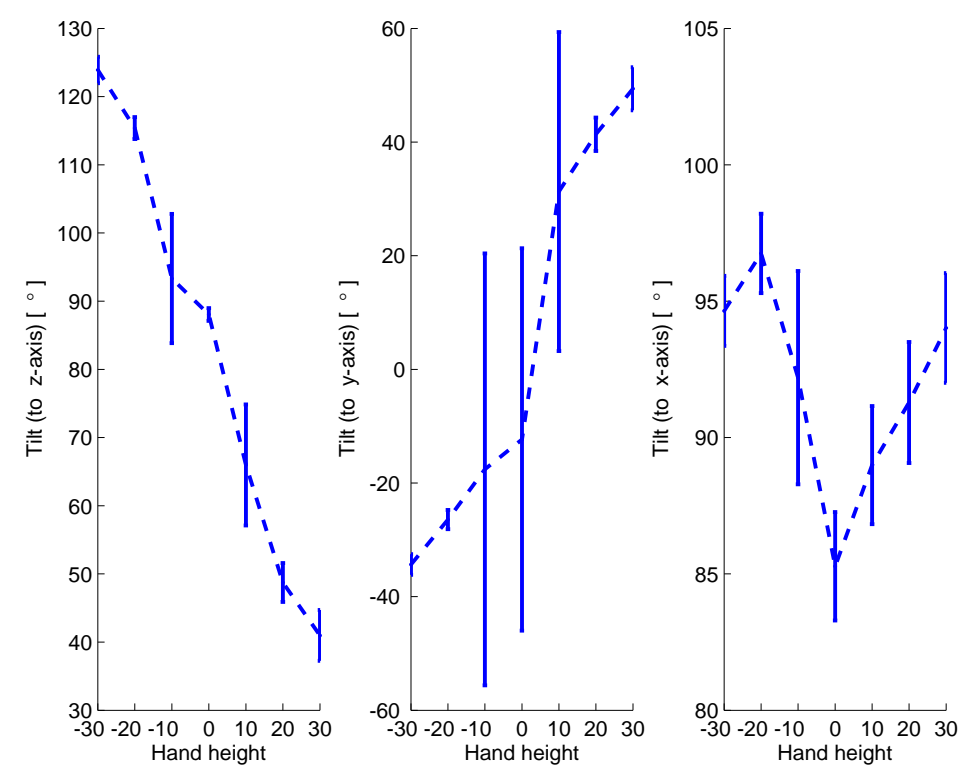

Figure 3.2: Tilt of endeffector stiffness with respect to the external coordinate frame. Subfigures contain angels to z-axis, $\mathrm{y}$-axis and $\mathrm{x}$-axis.

Figures 3.3 and 3.4 show the overall endeffector stiffness pattern of six naïve ${ }^{2}$ subjects.

\subsubsection{Symmetric versus antisymmetric stiffness component}

Table 3.1: Fractions norm of antisymmetric stiffness to norm of symmetric stiffness in two subjects who were aware of the special emphasis of posture maintenance in the experiment.

\begin{tabular}{|l|c|c|c|c|c|c|c|}
\hline Subject & $\begin{array}{c}\text { Centre } \\
-30 \mathrm{~cm}\end{array}$ & $\begin{array}{c}\text { Centre } \\
-20 \mathrm{~cm}\end{array}$ & $\begin{array}{c}\text { Centre } \\
-10 \mathrm{~cm}\end{array}$ & Centre & $\begin{array}{c}\text { Centre } \\
+10 \mathrm{~cm}\end{array}$ & $\begin{array}{c}\text { Centre } \\
+20 \mathrm{~cm}\end{array}$ & $\begin{array}{c}\text { Centre } \\
+30 \mathrm{~cm}\end{array}$ \\
\hline KF & 0.035 & 0.039 & 0.029 & 0.028 & 0.028 & 0.028 & 0.013 \\
\hline MS & 0.073 & 0.042 & 0.021 & 0.018 & 0.058 & 0.033 & 0.021 \\
\hline
\end{tabular}

For protocols the antisymmetric component of endeffector stiffness was computed, and its 2-norm was set in relation to the one of the symmetric stiffness.

\footnotetext{
${ }^{2}$ The experiment was done with distraction task to read English words from the screen. Since the probands' main focus lay on reading correctly and loudly, they were naïve about the strong motor control of the experiment.
} 

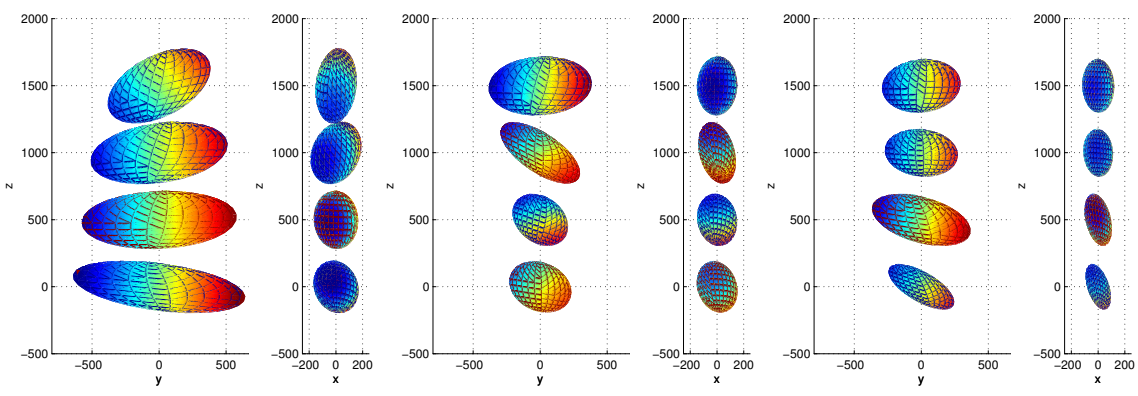

Figure 3.3: Endeffector stiffness along a short vertical line in front of the participant's shoulder. Studied hand positions were $10 \mathrm{~cm}$ apart, starting with a table height of 100 $\mathrm{cm}$. Left: Data from female participant. Colour scale of stiffness (ranging from blue to red): [-600, 600]. Middle: Data from another female. Colour scale of stiffness: [-400, 400]. Right: Data from male participant. Colour scale of stiffness: [-400, 400.].
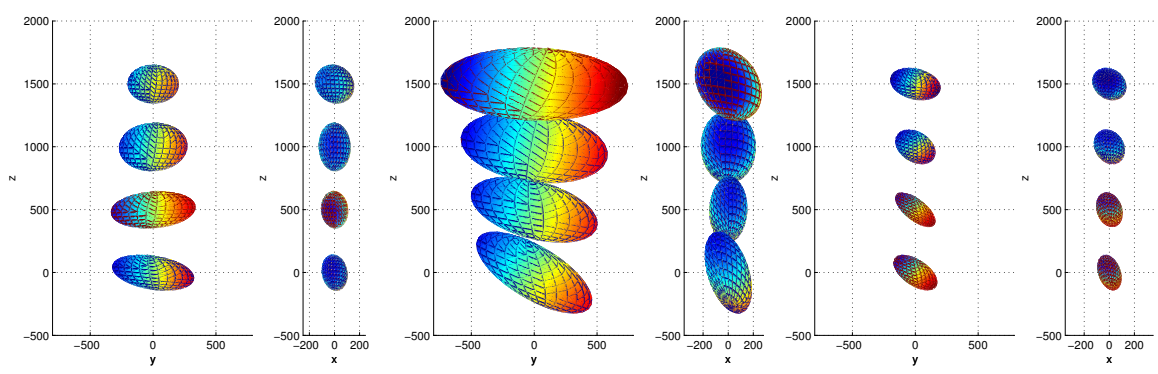

Figure 3.4: Endeffector stiffness along a short vertical line in front of the participant's shoulder. Studied hand positions were $10 \mathrm{~cm}$ apart, starting with a table height of 100 $\mathrm{cm}$. Data from three more female participants. Left: Colour scale of stiffness: [-400, 400]. Middle: Colour scale of stiffness: [-700, 700]. Right: Colour scale of stiffness: $[-200,200]$.

In general this fraction was low, indicating that the antisymmetric stiffness component is negligible (cf. Mussa-Ivaldi et al., 1985).

The overview of the fractions in 'well-informed' and 'naïve' subjects in Tables 3.1 and 3.2 shows an interesting difference. Participants who were not especially reminded of the importance of the postural task (but instead reinforced in the distraction task of reading English words from the screen) show a higher rotational component. This gives rise to the surmise that the antisymmetric part of stiffness can be voluntarily suppressed (at least to some extent) during this the measurement in the redundant arm. 
Table 3.2: Fractions norm of antisymmetric stiffness to norm of symmetric stiffness in six subjects who completed the experiment with emphasis on the word reading distraction task.

\begin{tabular}{|l|c|c|c|c|}
\hline Subject & $\begin{array}{c}\text { Table } \\
\text { at } 100 \mathrm{~cm}\end{array}$ & $\begin{array}{c}\text { Table } \\
\text { at } 110 \mathrm{~cm}\end{array}$ & $\begin{array}{c}\text { Table } \\
\text { at } 120 \mathrm{~cm}\end{array}$ & $\begin{array}{c}\text { Table } \\
\text { at } 130 \mathrm{~cm}\end{array}$ \\
\hline NP1 & 0.027 & 0.030 & 0.048 & 0.048 \\
\hline NP2 & 0.076 & 0.227 & 0.223 & 0.105 \\
\hline NP3 & 0.015 & 0.062 & 0.063 & 0.041 \\
\hline NP4 & 0.030 & 0.025 & 0.085 & 0.088 \\
\hline NP5 & 0.147 & 0.166 & 0.237 & 0.240 \\
\hline NP6 & 0.085 & 0.110 & 0.128 & 0.102 \\
\hline
\end{tabular}

\subsubsection{Hand stiffness in external force fields}

This section contains data about endeffector stiffness in constant external force fields. My aim was to show that the presence of such a force does not affect endeffector stiffness since it is compensated by additional torques at joint level (as suggested by Flash and Gurevich, 1991). That means calculating endeffector stiffness by using the applied probing forces and resulting hand deviations should lead to the 'same' stiffness estimates (with a reasonable variance) for different force conditions.

Figures 3.5 show stiffness estimates for two different hand positions (centre and $20 \mathrm{~cm}$ above centre) and three conditions of the external force field (bottom: $-3 \mathrm{~N}$, middle: $0 \mathrm{~N}$, top: $3 \mathrm{~N})$. Figures 3.6, and 3.7 show further stiffness estimates for the same force conditions applied to the hand in central position. Visual impression already shows a non-neglectable difference between the ellipses of the same participant. This indicated that with the given technique it is not feasible to obtain 'the same' endeffector stiffness for different external force fields. Variability in grip and posture, as well as a not completely fixed wrist (pronation/supination can not be fully suppressed with a cuff that is comfortably mounted at the hand) may account for this problem.

For a numeric test of the consistency of available data for a single hand position the data were split up by recording batches (two batches with three times all twelve displacements) and stiffness estimates $\bar{K}_{1}$ and $\bar{K}_{2}$ as well as according variances $s_{1}^{2}$ and $s_{2}^{2}$ were calculated by means of $\mathrm{LnO}(50)$. A t-test 
according to

$$
t=\frac{\bar{K}_{1}+\bar{K}_{2}}{\left(s_{1}^{2}+s_{2}^{2}\right) / 50} .
$$

results in the rejection of the assumption that the two stiffnesses are equal. Even though this states the obvious, the application of the t-test has to be read cum grano salis since splitting up the recorded data in two batches violates the previously shown limit of required data (cf. Section 2.3.2).

Further modelling, like extending the idea from Flash and Gurevich (1991) to the four-dimensional joint system, is not possible at this point. Even if it was, a detailed discussion was required concerning the results in the light of an error estimation for the transformation from hand to joint space. The rather coarse method of estimating joint angles from standardised photographs would cast doubt on the results. Other facts like

- different ways of grasping the handle of the machine,

- no full immobility of wrist rotation (cuff only impedes flexion/extension and abduction/adduction), and

- shoulder position can only be ensured with a precision (since the participant can easily modify it by eg. stretching her/his back) and is not monitored constantly (eg. by optical tracking or EMG) during trials

result in even further uncertainties about the joint angles during holding phase.
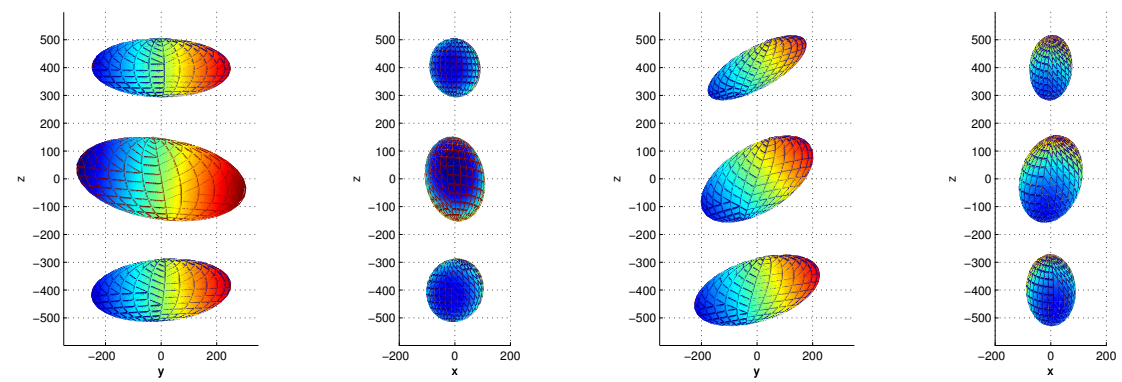

Figure 3.5: Estimated endeffector stiffness for one participant with arm in Left two plots: central position (6 repetitions). Right two plots: $20 \mathrm{~cm}$ above central position (3 repetitions). Height in plot corresponds to applied force strength. Force factor of displacements: 1.5. Colour scale left: blue -300, red 300. Left subplot: Side view. Right view: View towards subject. 

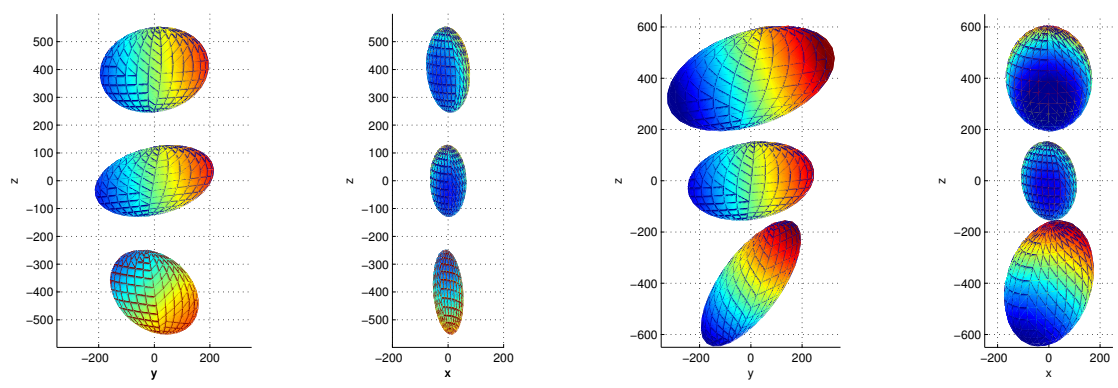

Figure 3.6: Estimated endeffector stiffness for two other participant with arm in central position (6 repetitions). Left two plots: female, centre position, $3 \mathrm{~N}$ external force field, force factor: 1.3. Right two plots: female, centre position, $3 \mathrm{~N}$ external, force factor 1.7. Colour scale left: blue -300 , red 300. Left subplot: Side view. Right view: View towards subject.
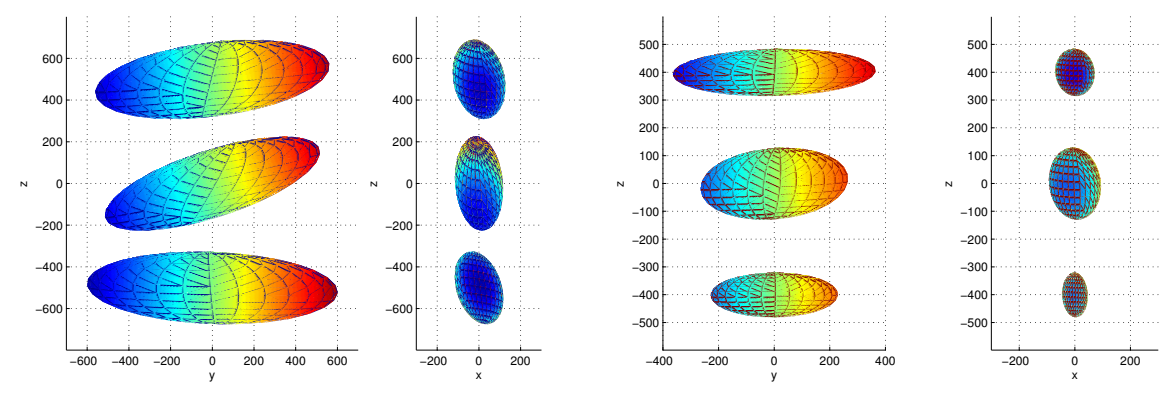

Figure 3.7: Estimated endeffector stiffness for two other participant with arm in central position (6 repetitions). Left two plots: male, centre position, 3N external force field, force factor 2.0. Right two plots: male, centre position, force factor 1.7. Colour scale left: blue -300 , red 300. Left subplot: Side view. Right view: View towards subject.

\subsubsection{Variation of hand stiffness with arm posture}

The idea of the posture variation protocol was to show the correlation between the rotation about the hand-shoulder axis for various hand locations and the respective endeffector stiffnesses.

During the definition phase of the postures at the beginning of the recording a comfortable posture $\vec{o}_{c}$ with the elbow at individually chosen height and an extreme posture $\vec{o}_{m}$ with the elbow at maximum height were set up. The participant had to get into these postures eleven times. Table 3.3 shows the precision of these first accommodation trials in terms of tilt and twist (cf. Section 2.2.4) of the handle compared to the fixed external coordinate frame. 
Figure 3.8 shows the average handle orientations produced at the time short before the onset of the displacement. The linear change in tilt and twist of the handle verifies the presented procedure of assessing the rotation about the hand-shoulder axis. While the direction of the handle is reproduced consistently (low variation in tilt with respect to the $\mathrm{x}$-axis of external coordinate frame) due to the experimental condition for the start of a trial (cf. Section 2.2.1), the rotational component of the handle showed a greater variance. This is mainly caused by changes between single recording batches when the participant releases the handle.

Table 3.3: Handle orientation (tilt $\phi$, twist $\xi$ ) during production of comfortable $\vec{o}_{c}$ and maximum posture $\vec{o}_{m}$ in eleven accommodation trials. The external coordinate frame is taken as a reference.

\begin{tabular}{|c|c|c|}
\hline & $\vec{o}_{c}$ & $\vec{o}_{m}$ \\
\hline$\phi$ & $126.61^{\circ}$ & $64.56^{\circ}$ \\
& $\pm 16.33^{\circ}$ & $\pm 3.42^{\circ}$ \\
\hline$\xi$ & $70.27^{\circ}$ & $5.72^{\circ}$ \\
& $\pm 22.32^{\circ}$ & $\pm 1.83^{\circ}$ \\
\hline
\end{tabular}

Figure 3.8: Average handle orientation at the start of the displacements in terms of tilt (angle with $\mathrm{x}$-axis of external coordinate frame) and twist (rotation around $\mathrm{x}$-axis).
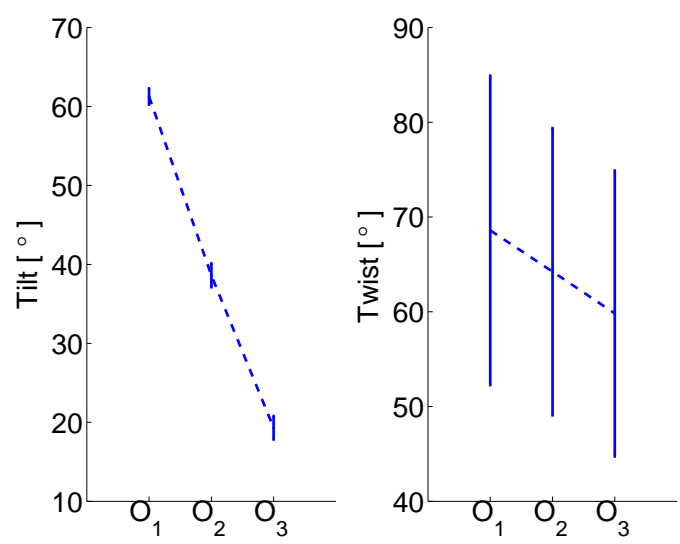

Due to the enormous muscular demands during the highly repetitive postural task, recordings could only be successfully finished for one participant. Subjects' ability to consistently reproduce the challenging postures was limited due to fatigue. In the following this data set is discussed.

Table 3.4 summarises variability in handle orientation during holding phase in dependence on displacing force direction. Handle orientations at the start of the displacement were taken as reference such that the data comprises the effect of the displacement on posture. It can be seen that all changes in handle orientation during the trial are negligible.

The change in the orientation of the estimated stiffness ellipsoids with recording posture can be seen in Figure 3.9. The left upper subfigure indicates that the 
Table 3.4: Relative discrepancy (tilt $\phi$, twist $\xi$ ) between handle orientations at start of the displacement and during the holding phase. Variation in $\phi$ and $\xi$ serves as a measure of consistency of posture during displacement. The size of $\phi$ and $\xi$ reflects the effect of the displacement on posture.

\begin{tabular}{|c|c|c|c|}
\hline$\phi$ & $\vec{o}_{1}$ & $\vec{o}_{2}$ & $\vec{o}_{3}$ \\
\hline$F_{1}$ & $0.016^{\circ} \pm 0.053^{\circ}$ & $0.025^{\circ} \pm 0.083^{\circ}$ & $0.013^{\circ} \pm 0.045^{\circ}$ \\
\hline$F_{2}$ & $0.026^{\circ} \pm 0.085^{\circ}$ & $0.015^{\circ} \pm 0.049^{\circ}$ & $0.067^{\circ} \pm 0.224^{\circ}$ \\
\hline$F_{3}$ & $0.011^{\circ} \pm 0.036^{\circ}$ & $0.021^{\circ} \pm 0.069^{\circ}$ & $0.027^{\circ} \pm 0.089^{\circ}$ \\
\hline$F_{4}$ & $0.018^{\circ} \pm 0.061^{\circ}$ & $0.055^{\circ} \pm 0.182^{\circ}$ & $0.046^{\circ} \pm 0.153^{\circ}$ \\
\hline$F_{5}$ & $0.011^{\circ} \pm 0.038^{\circ}$ & $0.015^{\circ} \pm 0.049^{\circ}$ & $0.007^{\circ} \pm 0.023^{\circ}$ \\
\hline$F_{6}$ & $0.016^{\circ} \pm 0.055^{\circ}$ & $0.013^{\circ} \pm 0.045^{\circ}$ & $0.020^{\circ} \pm 0.068^{\circ}$ \\
\hline$F_{7}$ & $0.016^{\circ} \pm 0.055^{\circ}$ & $0.040^{\circ} \pm 0.133^{\circ}$ & $0.094^{\circ} \pm 0.315^{\circ}$ \\
\hline$F_{8}$ & $0.025^{\circ} \pm 0.085^{\circ}$ & $0.024^{\circ} \pm 0.080^{\circ}$ & $0.056^{\circ} \pm 0.186^{\circ}$ \\
\hline$F_{9}$ & $0.007^{\circ} \pm 0.022^{\circ}$ & $0.050^{\circ} \pm 0.168^{\circ}$ & $0.054^{\circ} \pm 0.181^{\circ}$ \\
\hline$F_{10}$ & $0.070^{\circ} \pm 0.232^{\circ}$ & $0.013^{\circ} \pm 0.045^{\circ}$ & $0.052^{\circ} \pm 0.175^{\circ}$ \\
\hline$F_{11}$ & $0.012^{\circ} \pm 0.040^{\circ}$ & $0.004^{\circ} \pm 0.014^{\circ}$ & $0.021^{\circ} \pm 0.069^{\circ}$ \\
\hline$F_{12}$ & $0.021^{\circ} \pm 0.071^{\circ}$ & $0.010^{\circ} \pm 0.033^{\circ}$ & $0.016^{\circ} \pm 0.053^{\circ}$ \\
\hline
\end{tabular}

\begin{tabular}{|c|c|c|c|}
\hline$\xi$ & $\vec{o}_{1}$ & $\vec{o}_{2}$ & $\vec{o}_{3}$ \\
\hline$F_{1}$ & $0.009^{\circ} \pm 0.030^{\circ}$ & $0.023^{\circ} \pm 0.078^{\circ}$ & $0.015^{\circ} \pm 0.051^{\circ}$ \\
\hline$F_{2}$ & $0.013^{\circ} \pm 0.045^{\circ}$ & $0.025^{\circ} \pm 0.085^{\circ}$ & $0.021^{\circ} \pm 0.071^{\circ}$ \\
\hline$F_{3}$ & $0.006^{\circ} \pm 0.020^{\circ}$ & $0.014^{\circ} \pm 0.045^{\circ}$ & $0.011^{\circ} \pm 0.037^{\circ}$ \\
\hline$F_{4}$ & $0.020^{\circ} \pm 0.068^{\circ}$ & $0.018^{\circ} \pm 0.058^{\circ}$ & $0.015^{\circ} \pm 0.051^{\circ}$ \\
\hline$F_{5}$ & $0.008^{\circ} \pm 0.026^{\circ}$ & $0.024^{\circ} \pm 0.081^{\circ}$ & $0.012^{\circ} \pm 0.042^{\circ}$ \\
\hline$F_{6}$ & $0.004^{\circ} \pm 0.012^{\circ}$ & $0.020^{\circ} \pm 0.066^{\circ}$ & $0.025^{\circ} \pm 0.085^{\circ}$ \\
\hline$F_{7}$ & $0.012^{\circ} \pm 0.041^{\circ}$ & $0.014^{\circ} \pm 0.046^{\circ}$ & $0.033^{\circ} \pm 0.109^{\circ}$ \\
\hline$F_{8}$ & $0.023^{\circ} \pm 0.075^{\circ}$ & $0.015^{\circ} \pm 0.050^{\circ}$ & $0.025^{\circ} \pm 0.082^{\circ}$ \\
\hline$F_{9}$ & $0.009^{\circ} \pm 0.028^{\circ}$ & $0.024^{\circ} \pm 0.080^{\circ}$ & $0.024^{\circ} \pm 0.078^{\circ}$ \\
\hline$F_{10}$ & $0.015^{\circ} \pm 0.049^{\circ}$ & $0.020^{\circ} \pm 0.067^{\circ}$ & $0.020^{\circ} \pm 0.067^{\circ}$ \\
\hline$F_{11}$ & $0.016^{\circ} \pm 0.053^{\circ}$ & $0.007^{\circ} \pm 0.022^{\circ}$ & $0.012^{\circ} \pm 0.040^{\circ}$ \\
\hline$F_{12}$ & $0.001^{\circ} \pm 0.004^{\circ}$ & $0.012^{\circ} \pm 0.040^{\circ}$ & $0.010^{\circ} \pm 0.034^{\circ}$ \\
\hline
\end{tabular}


Table 3.5: Orientation of major axis of stiffness in dependence on elbow elevation (determined by handle orientation).

\begin{tabular}{|c|c|c|c|c|c|c|c|}
\hline Elbow & $\begin{array}{l}\text { Angle } \\
\text { with } \\
\text { x-axis }\end{array}$ & $\begin{array}{l}\text { Angle } \\
\text { with } \\
\text { v-axis }\end{array}$ & $\begin{array}{l}\text { Angle } \\
\text { with } \\
\text { z-axis }\end{array}$ & $\begin{array}{c}\text { Elbow } \\
\text { pos. }\end{array}$ & $K_{\max }$ & $K_{\text {med }}$ & $K_{\min }$ \\
\hline upper & $93.65^{\circ}$ & $76.19^{\circ}$ & $14.29^{\circ}$ & \multirow{5}{*}{$\begin{array}{l}\text { middle } \\
\text { down }\end{array}$} & 462.09 & 134.51 & 126.19 \\
\hline & & & & & \pm 25.33 & \pm 4.87 & \pm 4.00 \\
\hline middle & $97.72^{\circ}$ & $80.93^{\circ}$ & $11.95^{\circ}$ & & $\begin{array}{r}484.24 \\
+\quad 3380\end{array}$ & $\begin{array}{r}145.48 \\
+647\end{array}$ & $\begin{array}{r}106.94 \\
+\quad 568\end{array}$ \\
\hline & & & & & 430.84 & 140.30 & 122.99 \\
\hline down & $78.32^{\circ}$ & $98.14^{\circ}$ & $165.69^{\circ}$ & & \pm 26.79 & \pm 5.65 & \pm 4.44 \\
\hline
\end{tabular}

Table 3.6: Eigenvalues of symmetric endeffector stiffness with constant hand position for various elbow heights.

angle between the major axis of the stiffness and the external coordinate system rises in relation to z-axis and drops referring to the x-axis when increasing the angle between the orientation of the handle and the external coordinate frame (lifting the elbow). So stiffness direction is influenced by posture in a consistent way. The twist of the handle remains about the same throughout postures. The lower part of Figure 3.9 shows that also the orientation between major axis of stiffness and handle changes with posture. So the stiffness ellipsoid is not just 'dragged' to another orientation because of being fixed to the handle or the arm but changes throughout workspace. Since all other joint angles remain the same, this effect may result from the redundancy in the arm. This opens the interesting possibility of assessing joint stiffness by studying its traces in the variation of the hand stiffness components, if it can be accomplished to record in more participants different hand locations in the daily workspace. 
Outer tilt of stiffness to outer tilt of handle

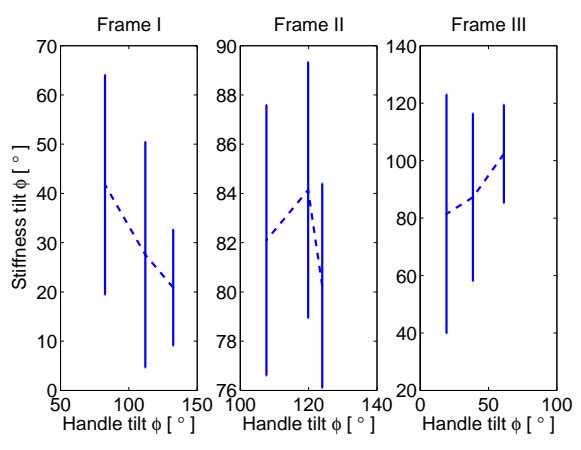

Tilt of orientation difference to outer tilt of handle

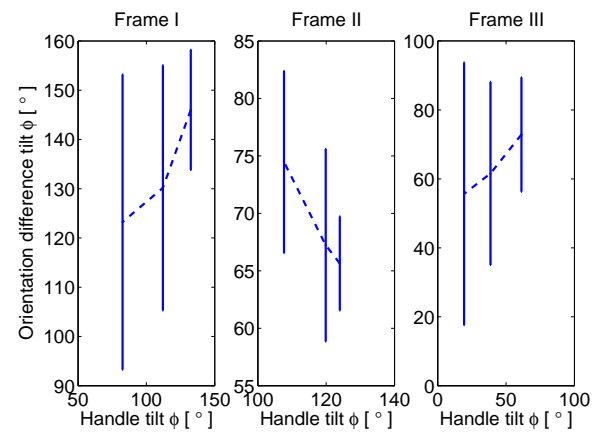

Outer twist of stiffness to outer twist of handle

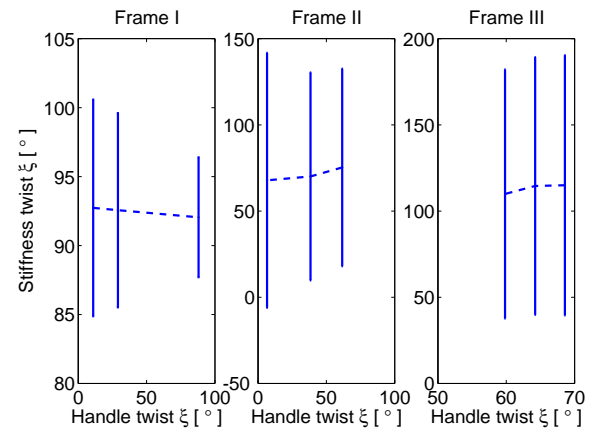

Twist of orientation difference to outer twist of handle

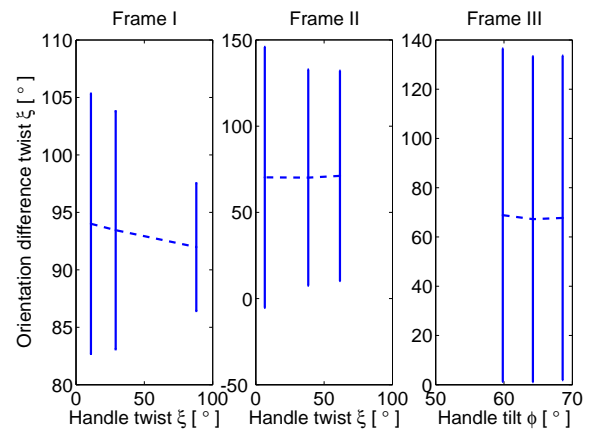

Figure 3.9: Upper: Relation of average orientation of stiffness ellipsoid to average handle orientation, both in terms of outer tilt and outer twist (with respect to the external coordinate frame). Subsubfigures from left to right: Frame I: normal coordinate frame (twist in $\mathrm{x}$ - plane, z-axis used for tilt computation). Frame II: y-axis serves for tilt. Frame III: x-axis used for tilt computation. Lower: Relation of average orientation difference between major axis of stiffness ellipsoid and average handle orientation to average handle orientation (both in terms of tilt and twist with respect to the external coordinate frame). Subsubfigures from left to right: Frame I: normal coordinate frame. Frame II: y-axis serves for tilt. Frame III: x-axis used for tilt computation. 

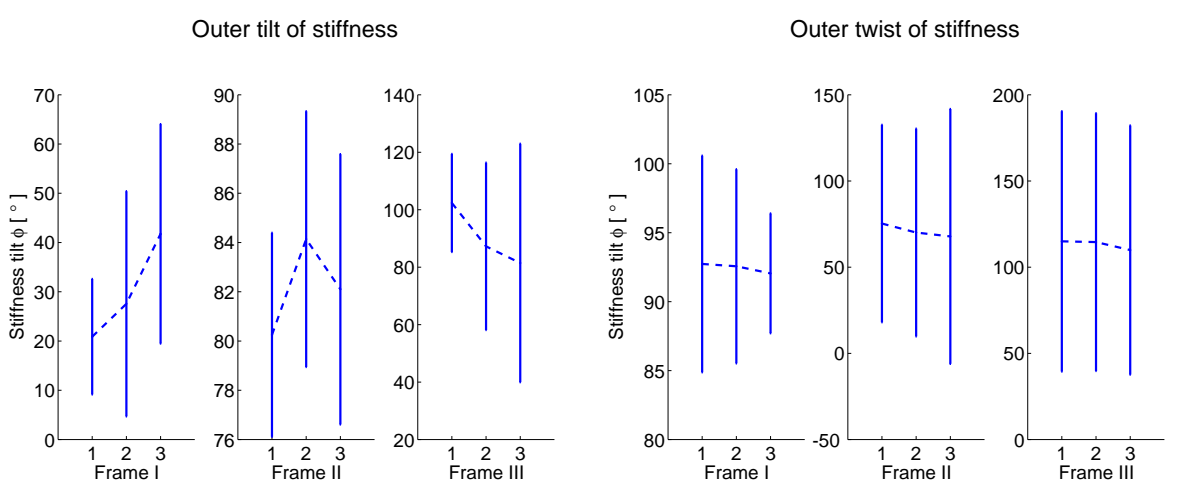

Inner tilt of stiffness

Inner twist of stiffness
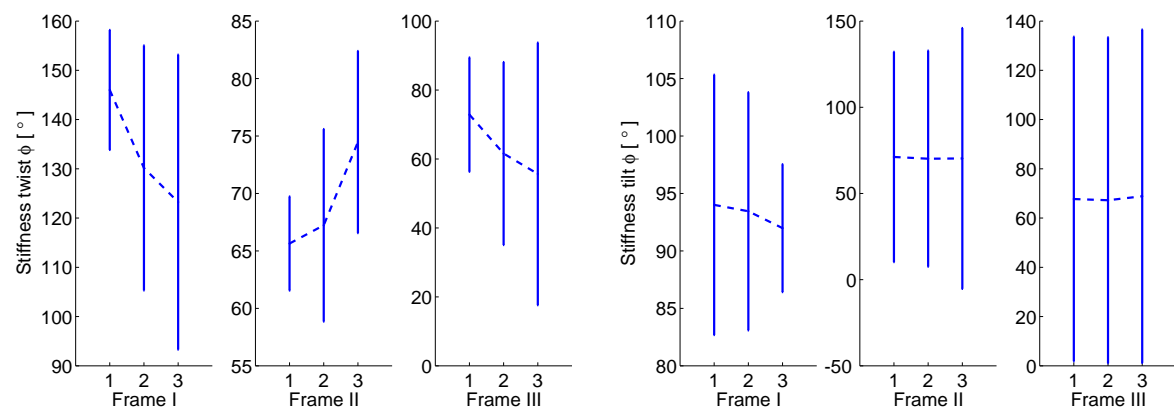

Figure 3.10: Upper: Average orientation of stiffness ellipsoid in terms of tilt and twist. Frame I: normal coordinate frame. Frame II: y-axis serves for tilt. Frame III: $\mathrm{x}$-axis used for tilt computation. Lower: Average orientation difference between stiffness ellipsoid and average handle orientation in terms of tilt and twist. Frame I: normal handle coordinate frame. Frame II: handle y-axis serves for tilt. Frame III: handle $\mathrm{x}$-axis used for tilt computation. 


\subsection{Discussion}

The developed method for endeffector stiffness measurements in the redundant arm in postures of the natural workspace using small force-induced hand displacements was proven to be successful in producing consistent results in agreement with classical results.

\subsubsection{Technical and individual challenges}

Technical problems like tuning of the strength of the displacing force and its ramping time were solved effectively. Further challenges like the reliable reproduction of grasping the handle and the complete immobilisation of forearm pronation/supination had to be solved at the individual level of the participants and could be only monitored to some extent. In the end, this led to variability in arm position which cannot be neglected in its effects on arm stiffness.

\subsubsection{Recording time as a practical limit}

The usage of the specific manipulandum which only could produce small forces and was not usable for PD-controlled displacements ${ }^{3}$ caused long session durations. Well-trained participants could handle three sets of the twelve displacing forces in ten minutes, and needed an intersession break of between three and five minutes. Yet the majority of probands needed about 15 minutes to complete the same number of trials. Intersession breaks were usually also longer (up to 10 minutes), and additional breaks of about two minutes were taken during recording time. So between 26 and 50 minutes were needed to accumulate enough data for one experimental condition in one hand location.

\subsubsection{Reproduction and extension of classical results}

The independence of stiffness from constantly acting external force fields could not be shown consistently. The reduced data quality (cf. Section 3.3.4) set limits to further tracing of the origin of this problem. It might be that the compensation of additionally arising torques at joint level could not be compensated at single joint level due to muscle configuration, so that results different from the classical ones would have to be expected. A further

\footnotetext{
${ }^{3}$ Non-controllable oscillations at different frequencies, which changed with the participants' level of muscular cocontraction, lead to force kicks that would have harmed the hardware, if they were repeated too often.
} 
explanation could be that the applied measurement technique caused artifacts due to the redundancy of the system.

We demonstrated successfully the position and posture dependence of endeffector stiffness. Contradictory to results of studies in a horizontal plane at shoulder height we found that the direction of highest stiffness was aligned with the forearm direction (which coincides with the latest findings of Krutky et al. (2010)), suggesting a low effect of elbow stiffness and two-joint muscle contribution, while a higher stiffness was kept at the shoulder. So interactions with the environment predominantly induced displacements at elbow level. Assuming this as a general principle the weak immobilisation of elbow pronation/supination along with the muscle configuration at the forearm could be considered crucial for the problems in showing consistent compensation of external force fields.

\subsubsection{Distinguishability of positions}

Although Figure 3.1 is quite suggestive in terms of variation of endeffector stiffness with hand position, statistical discrimination using a t-test according to Equation 3.1 failed. Furthermore splitting up the ten repetitions of one hand position in the long line experiment into two data batches and comparing resulting stiffnesses also led to the judgement that data could not originate from the same distribution. The above mentioned individual challenges strongly contributed to this variability. This fact limits the usability of the achieved data in the modelling context, where clearly distinguishable data sets of joint stiffnesses would be needed. A planned extension of the geodesic model from Biess et al. (2007) was cancelled up for this reason.

\subsubsection{Summarising judgement of the method}

The presented method allows us to qualitatively study endeffector stiffness in virtually all arm postures of the daily workspace. Unfortunately, when it comes down to compete in accuracy with established methods sufficient precision cannot be assured in terms of convincing p-values, given the ethical limits (to session durations) of motor control experiments in humans. Nevertheless, the technique is applicable to gaining general results such as the forearm alignment of end-

effector stiffness. These insights can be used for conclusions about strategies which may be implemented in the central nervous system for interactions with the environment. 
$\sqrt{\text { conseres }} 4 \square$

\section{An experimental framework for} movement studies

\subsection{Introduction}

In course of a day humans produce a vast variety of arm postures and movements. To systematically study specific features of the planning and execution process, this multitude of possibilities has to be narrowed down by presenting a well-defined task where possible variables influencing the process of interest are controllable.

For this purpose $\mathrm{we}^{1}$ designed a configurable virtual reality setup that can be utilised for a wide range of motor control studies. The hardware comprises a robot manipulator, called manipulandum, that can track hand positions and orientation in three-dimensional space at a very high precision, as well as exert forces to the hand of the participant. Furthermore, the setup contains a computer screen for visual feedback.

Software control elements were wrapped up in a toolbox. Its key element, which is the so-called "Simulator", provides an interface for the configuration of three-dimensional virtual parcours that can contain movement targets, obstacles and configurations of surrounding walls. Properties such size, shape, color, as well as visibility and haptic rendering of the virtual objects, and changes of these properties in time can be specified using configuration files written in a

\footnotetext{
${ }^{1}$ Alexander Wolf prepared the ground for some of the presented experiments by conducting valuable pre-studies.
} 
script language developed by us, or using a user-friendly graphical user interface which we designed for quick online interaction with the setup any time during the experiments.

Virtual objects and obstacles can be presented at specific positions on the computer screen with different timings to study via-point and obstacle avoidance movements. Generated data then can be analysed with respect to timing, trajectory characteristics, or any other features of interest.

After a general introduction of the developed framework for movement studies in three-dimensional space I present a specific utilisation of the setup for a two-alternative forced-choice task. The aim of this task is to examine how humans handle motor decision tasks under subjective time pressure. For this purpose trajectory formation and error correction is analysed in Chapter 5, and an "event-based" study of decision tendencies and preferences is provided in Chapter 6.

Further technical details about the experimental framework can be found in Appendix A.4.2, and give an idea of the wide range of experimental schemes that are possible with the presented environment

\subsection{General methods}

\subsubsection{Setup overview}

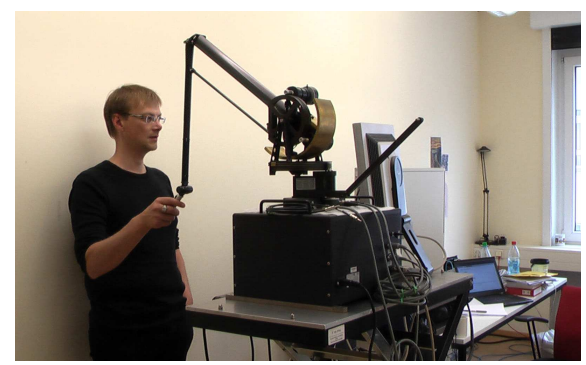

(a) Participant in front of the hardware setup including robot manipulator and computer screen during conduction of the experiment.

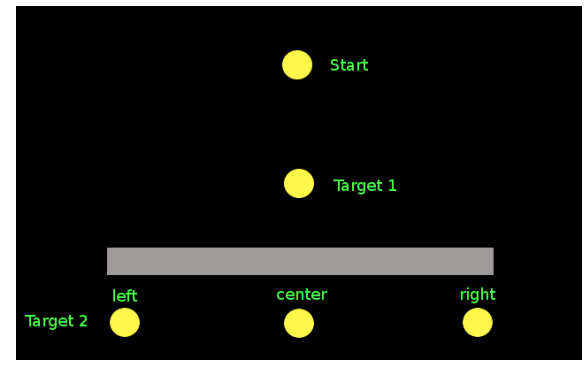

(b) Scheme of an experiment designed with the Simulator. The figure shows all possible target locations along with their names which are used throughout this thesis.

Figure 4.1: Overview of the setup and of the specific parcours used for the subsequent studies. 
The setup was designed to study human arm movements in a set of tasks which are inspired by everyday movements. It is configurable to pose a large variety of motor task, such as point-to-point movements, via-point movements, obstacle avoidance tasks, mace tasks, as well as static or "dynamic" combinations of them. In this context, dynamic refers to changes of the target and obstacle configuration over time.

The system hardware comprises a robot manipulandum and a computer screen, which both are placed on a height-adjustable laboratory table. The software for configuring and running the experiments is wrapped up in the socalled Simulator. It provides a configurable virtual reality parcours which can be displayed on a computer screen (or on head-mounted displays, if visual feedback of the arm should be excluded). The positions of targets and obstacles in the three-dimensional (work-) space can be defined comfortably using a custommade setup script language (see Appendix A.4.2 for specification). An example parcours is displayed in Figure 4.1(b).

\section{Machinery}

For executing movements in the parcours, the participants interact with a high performance manipulandum (SensAble Phantom 3.0 6DoF, see Appendix A.2 for technical details). They grasp a handle which is attached to a light-weighted mechanical arm that can move freely and quasi-frictionless in three-dimensional space. Additional three degrees of freedom allow a wide range handle rotations with respect to the machine arm. Hand movement trajectories are recorded by tracking a well-defined point of the handle, so that in general tracking precision in sub-millimetre range is possible. ${ }^{2}$ Besides tracking, the manipulandum is furthermore utilised to haptically render surfaces of virtual walls and obstacles using a spring-like simulation of contact forces. At each instant of time ${ }^{3}$ the force exerted to the hand is proportional to the distance the virtual representation of the hand already moved into the according object.

The manipulandum has a preferred working range of $45 \mathrm{~cm}$ to the left and to the right, $90 \mathrm{~cm}$ upwards from the point of initialisation ${ }^{4}$, and $15 \mathrm{~cm}$ towards the participant as well as $15 \mathrm{~cm}$ towards the table. In this range all servo motors

\footnotetext{
${ }^{2}$ This precision refers to tracking the gimbal joint of the manipulandum. Only under completely determined circumstances (especially in the way of grasping the handle) the values can be transformed into an exact hand position. This kind of highly precise tracking was not necessary for the movement studies presented in Chapters 5 and 6 .

${ }^{3}$ The haptic loop runs at a frequency of $1000 \mathrm{~Hz}$.

${ }^{4}$ In the initialisation posture all joints of the machine are at 90 degrees.
} 
reliably produced the commanded forces. This range (or any sub-range of it) is called workspace in the following.

\subsubsection{Details on virtual reality}

The workspace is mapped to a three-dimensional visual representation on a scale of 1:2 and displayed on a computer screen by means of central perspective (usage of OpenGl) to provide visual feedback of setup to the participant. The $\mathrm{x}-\mathrm{y}$ plane of this virtual space is parallel to the plane of the screen. Borders of the workspace are rendered visually as light-grey walls and haptically by force production when a hand position "behind" the wall was detected. The participant's hand is represented by a blue sphere in the virtual parcours. The position of this feedback sphere is updated at $250 \mathrm{~Hz}$, so the movement is perceived as continuous. For technical purposes distance in the virtual world is measured in "units". One unit corresponds to a distance of $25 \mathrm{~cm}$ in the real world.

Movement targets are represented by coloured spheres on the screen. Their size is adjustable in the configuration script (see Table A.4 in Appendix A.4.2). The default size is 0.04 units.

For subsequent studies I constrained the the working range of the manipulandum to a box of $50 \mathrm{~cm} \times 50 \mathrm{~cm} \times 3.75 \mathrm{~cm}$ around the centre of the box of optimal performance, since I was only interested in quasi-planar trajectories. The borders of the corresponding bounding box in the virtual setup were defined as -1 and 1 in $x$ - as well as in y-direction. Furthermore, invisible walls were inserted at -0.075 and 0.075 units in z-direction. The rectangular obstacle used for the presented studies had a size $1.2 \times 0.05$ units $(30 \mathrm{~cm} 1.25 \mathrm{~cm}$ in endeffector space) and was located in the lower half of the screen (cf. Figure 4.1(b)).

\subsubsection{Simulator}

The Simulator toolbox for the experiment is written in $\mathrm{C}++$ and comprises a world editor, a target positioner and randomiser, a protocol editor, and a compilation of analysis scripts.

World editor This set of $\mathrm{C}++$ classes provides means to create a block world setup. All basic properties of the objects can be set here. Objects in the virtual world can be rendered visually and haptically ${ }^{5}$. Graphical rendering

\footnotetext{
${ }^{5}$ To increase the variety of applications these rendering modes are optional.
} 
is done using OpenGl. Haptic properties of the virtual objects are simulated using collision detection routines from the OpenHaptics library operating the Phantom manipulator in the HL mode ${ }^{6}$.

Target positioner After definition of a number of world objects their positions and timing of occurrence can be scheduled using the methods of the target positioner. Further properties, such as colour and size, are also accessible. The randomiser provides means to create random time series of target locations with various statistical properties. It produces a sequence of locations of all non-stationary targets given an initial specification of limiting values (cf. Appendix A.4.2 for details). Further object properties can also be altered randomly.

Protocol editor The protocol edition contains a number of predefined experiments and provides the option to adjust all session parameters manually using a custom-made script language (see Appendix A.4.2 for an example protocol).

Data analysis scripts Beside trajectory and timing information, the data accumulated throughout sessions contains specific markers that indicate important events, such as arriving in a or leaving a target. Typical steps of data analysis are marker extraction and calculation of average movement durations are summarised in a number of shell scripts. For the analysis of via-point movements with three targets a further compilation of scripts was written. A number of shell scripts, which can run on the lab machine while the participants do their experiments, help to get a quick overview of the individual performance ${ }^{7}$ in course of a session and on average. A more detailed data analysis can be carried out using a number of MATLAB scripts which I wrote for via-point movements with three targets.

\footnotetext{
${ }^{6} \mathrm{HL}$ denotes "high-level" operation mode. In this mode hardware-related issues as well as scheduling necessary control loops on the operating system are hidden from the user, who can only use a small number of specific functions for direct interaction with the machine.

${ }^{7}$ Performance measures are, for example, trial completion time, timing of and peak velocities at critical points in the setup, and, if an obstacle is presented, the choice of movement direction around the obstacle.
} 


\subsection{Specific protocol for subsequent studies}

\subsubsection{Framework of the "Probability experiment"}

The studies presented here were based on the so-called probability experiment. The workspace contained one obstacle and three movement targets. The participants had to start their trials in a fixed starting position, and then move through a via-point T1, around the obstacle towards a final target T2. T2 was not visible at the beginning of a trial, but appeared on arrival in $\mathrm{T} 1$ in specific locations behind the wall. For the presented studies only two T2 locations were used, one on the left behind the wall, and one on the right. In this experimental protocol the probability of T2 to occur in any of these spots can be defined, along with the option to hide T2 until T1 is reached. The average probability of $\mathrm{T} 2$ to occur on either side was set to 0.45 . The remaining 10 percent were used for "catch trials" where T2 was placed in the middle behind the obstacle.

\subsubsection{Specification of a trial}

Given the above framework, we defined a trial with the following restrictions: The participant had to move from a starting point through an intermediate target to a final point T1 passing the obstacle at that side which caused the shortest trajectory. Revealing the location of the final target just upon arrival in the intermediate point caused some uncertainty that had to be bridged by a 'good guess'.

Screenshots of a typical trial are depicted in Figure 4.2A-D. Workspace borders (not in the figure) and the obstacle were displayed all the time. The subfigures illustrate the following steps of the trial:

0. Initial setup: The starting point and the first movement target T1 were displayed. The participant had to move the hand to the starting position.

1. On correct positioning of the hand in the starting position: After a random delay the starting sphere turned green. A movement towards target T1 had to be initiated. There was no time limit.

2. On arrival in T1: The target sphere turned green, and the location of target T2 was revealed: randomly either left or right behind the wall, or at the centre in 10 percent catch trials. A time limit counter was started 
and a rectangular region of $0.8 \times 0.08$ units around $\mathrm{T} 1$ had to be left within the next $350 \mathrm{~ms}^{8}$

3. Successful completion of the trial: The participant moved without any long breaks to target $\mathrm{T} 2$, passing the obstacle at the correct side.

A trial was considered valid (or successful), if the time restriction in T1 was met and the shortest way around the obstacle was taken, and invalid (or unsuccessful) otherwise. Furthermore, trials with failing to catch target T1 and proceeding around the wall had to be repeated.
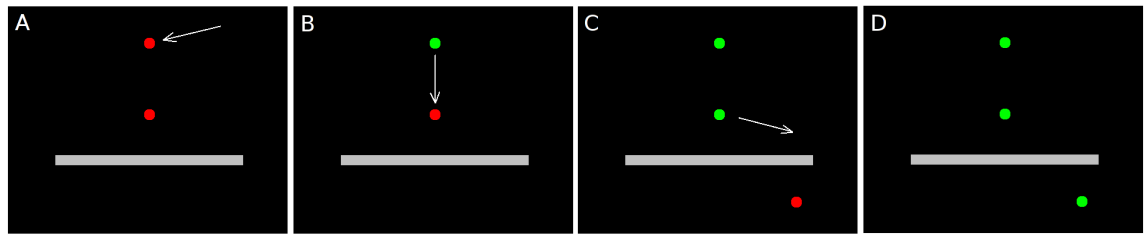

Figure 4.2: Visual feedback of a typical trial. White arrows were just added for sake of illustration here. A: Get into starting position. B: Move through target T1. C: Continue moving around the obstacle. D: Successful arrival in target T2.

The locations of target $\mathrm{T} 2$ were computed based on random numbers $r$ between 0 and 1 . If the number was smaller than the "neutral probability" $p_{n}=0.1$, target $\mathrm{T} 2$ was placed in the centre behind the wall to produce a catch trial. If $r$ was larger than $p_{n}$ but smaller than $p=0.65 \mathrm{~T} 2$ is placed on the left side behind the wall. Otherwise it was placed on the right. ${ }^{9}$

For each trial the trajectory, and the timing of important events, such as leaving the start position $\left(t_{S}\right)$, arriving in target $\mathrm{T} 1\left(t_{T 1}\right)$, passing the wall ${ }^{10}$ $T_{W}$ and arrival in target $\mathrm{T} 2\left(t_{T 2}\right)$, were recorded.

\subsubsection{Technical details}

Participants could choose whether to sit or stand in front of the setup (manipulandum and computer screen) which was placed on a height-adjustable table. Standing was defined as standing freely in a neutral upright posture in front of the setup, sitting was defined as sitting on a chair with a backrest and low

\footnotetext{
${ }^{8}$ Participants were not aware of the shape of this penalty window even when trying to find it out.

${ }^{9}$ In general all (logically consistent) desired combinations of $p_{n}$ and $p$ (within $[0,1]$ ) are possible.

${ }^{10}$ which is passing its $\mathrm{y}$-coordinate
} 
arm rests that do not interfere with any of the movements. The manipulandum was always positioned in front of the active arm so that no occlusions of the screen occurred during movement. The average distance of the eye plane to the computer screen was $85 \mathrm{~cm}$.

All via-point movements were executed in a quasi-frontoparallel ${ }^{11}$ plane which at the same time was parallel to the feedback screen (cf. Figure 4.1(a)). Hand positions were recorded in three-dimensional space at $90 \mathrm{~Hz}$ along with a time stamp.

\subsubsection{Experimental procedure}

Four session with 250 successful trials each were recorded for both the dominant and the non-dominant hand of all participants. For intersession and intersubject comparison in each session the same sequence $s$ of T2 locations was used. It was chosen specifically to contain a mild initial overrepresentation of $\mathrm{T} 2$ at the right side. For a better understanding of the time course, Figure 4.3(a) shows the time course of the instant directional bias (relative frequency of right minus relative frequency of left presentations; for details see Section 6.3.1), accumulated over the whole time series (green graph), as well as computed for each time step based

on the data of the last 30 trials (red graph). While the accumulative averaging reveals the overall tendency, the local averaging emphasises local fluctuations. It can be seen that there is an initial 'overrepresentation of right' during the first 50 trials. For a balanced presentation, the "overhang" is compensated later on by a stronger emphasis of $\mathrm{T} 2$ positions on the left with up to 11 repetitions around trial 140 and 8 repetitions around trial 250 (see Figure 4.3(b) for counts of occurring sequence lengths).

Since local fluctuations in the series of presented T2 location turned out to be crucial, I introduced two experimental conditions:

initR refers to the initial overrepresentation stated above, and indicates the usage of the series $s$ as it is shown in Figure 4.3, and

initL stands for an inversion of the same sequence ("left" and "right" swapped), and contains an initial overrepresentation of the left T2 location.

Both conditions were used in participants of either handedness.

\footnotetext{
${ }^{11}$ Since subjects are not fixated to anything in the lab, their upper body orientation can only be called approximately parallel to the screen.
} 


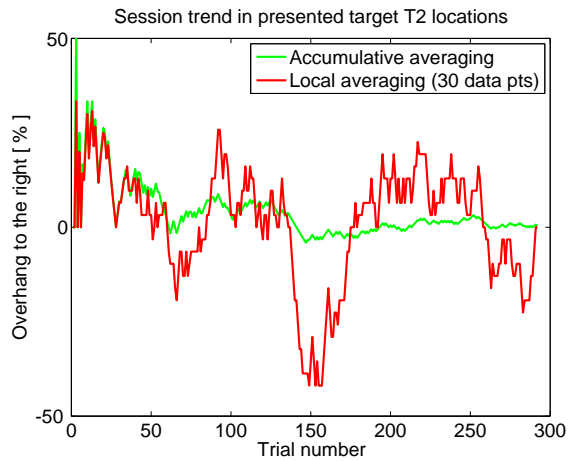

(a) 'Overhang' in presentation of T2 at the right behind the wall (green graph - accumulative averaging, red graph - local averaging with 30 data points window size).
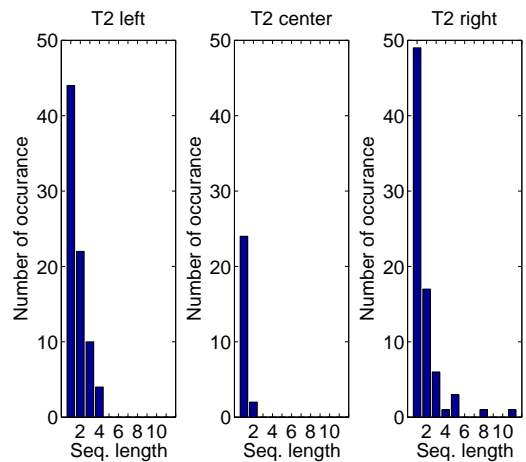

(b) Counts of sequences of subsequent occurrences of $\mathrm{T} 2$ presentations at same side - left, centre, or right - behind the obstacle. ("Seq. length" indicates the length of the sequence of repetitions.)

Figure 4.3: Statistical properties of the presented series of final target locations of the experimental condition initR. The time course of differences between relative fraction of right and the relative fraction of left behind the wall presentations of target T2 show an initial overhang to the right (first 50 trials). Nevertheless, there were two long sequences of $\mathrm{T} 2$ being positioned on the left side behind the obstacle.

\subsection{Participants}

\section{Participants}

Sixteen healthy adults (aged 19 to 42) with normal or corrected to normal vision participated in the experiments. Eight of them were left- and eight righthanded. ${ }^{12}$ They were assigned to two groups, such that four probands of each handedness participated in the experiments based on initR, and initL, respectively. All participants performed four sessions with their dominant and four with their non-dominant hand.

The completion of 259 valid trials usually took between 13 and 22 minutes. Participants had to take a break of at least five minutes between sessions, but were free to extend it if they needed more time.

They were encouraged to state their thoughts about the experiment freely. This should prevent them from developing silent hypothesis.

For a clear discussion of individual behaviour in the following chapters abbreviations containing two upper-case letters are used to distinguish probands.

\footnotetext{
${ }^{12}$ Before starting the experimental session they were asked to estimate their hand-eye coordination on a scale between 1 (completely uncoordinated) and 10 (perfect coordination).
} 


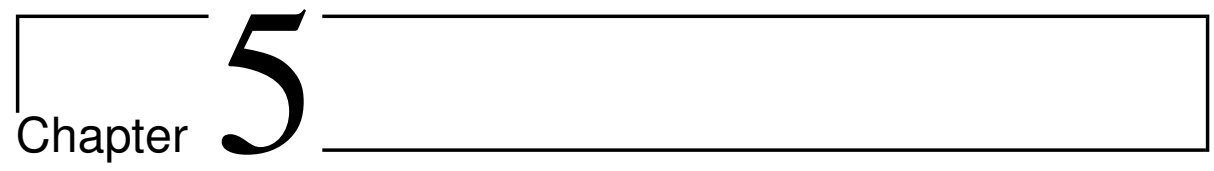

\section{Planning and replanning of fast} movements

\subsection{Introduction}

During the experiments, which I conducted, participants had to carry out dozens of via-point movements with obstacle avoidance. Their movements were recorded in terms of hand positions in three-dimensional space along with a time stamp. These trajectories are subject to change during the process of accommodation with the setup. Afterwards they can be considered as relatively constant. Their timing, especially the timing of correcting movements in case of an erroneous decision where to move, can provide important insights into movement planning.

Therefore, this chapter deals with a study of trajectory formation in the introduced setup for via-point movements with obstacle avoidance, and analyses direct movements where the shortest way to the final movement target was chosen immediately after $\mathrm{T} 1$, and replanned movements with an initial "kink" - or excursion - into the wrong direction.

The introductory Section 5.2 summarises major approaches to movement planning, trajectory formation and error correction. After some methodological comments in Section 5.3, I discuss the timing issues of the participants' movements in important stages of the task completion. For example, the processing 
time for the revealed location of the final movement target can be estimated by analysing data of trials containing a directional correction.

Then, single quasi-straight movement segments are modelled using bellshaped velocity profiles which are accepted "identifiers" - or at least correlates of movement primitives ${ }^{1}$ (cf. Plamondon et al., 1993; Flash and Hochner, 2005).

As described in Section 4.3.2 participants had only limited information about the final target location when having to make their decision about whether to pass a central obstacle at the left or right side. Based on the experience from previous trials and maybe based on temporary hypotheses about the occurrence of target T2 they had to make a guess. In a non-neglectable number of trials this guess was wrong ${ }^{2}$.

After an initial start to the wrong side the movement was corrected by a return. This correction is also modelled using symmetric continuous bell-shaped velocity profiles. First, the initial phase of correct movements to either side was modelled accordingly to extract the corresponding 'primitive', and second, the correction was introduced as a superposed 'primitive' which is started at a time $t_{\text {crit }}$ after the onset of the erroneous movement. $t_{\text {crit }}$ turned out to be comparable to the 'auto-pilot' threshold (cf. Pisella et al. (2000)), which is the time when a jump in location of the movement target is visible in the trajectory of the executed movement. This suggests that similar mechanisms are involved, and opens the door for follow-up studies of replanning interruptions using transcranial magnetic stimulation targeting on specific circuitry of the posterior parietal cortex known be involved in the online control of visually guided movements.

Furthermore, peak velocities and timing of the wrong movements and according corrections allow to discuss the influence of handedness and used hand in dependence on movement direction. It turned out that neither handedness nor hand usage has any significant effect on replanning characteristics. Shape and timing of kinks to the left and the right were symmetric in both hands.

\footnotetext{
${ }^{1}$ Primitives are considered to be the basic building blocks of complex motor behaviour. This theory significantly reduces the amount of computation that the nervous system would have to carry out during movement planning and execution.

${ }^{2}$ See Section 6.4 .1 for a strategy to keep this number high to provoke corrections for further studies of the replanning process.
} 


\subsection{Trajectory formation and motor primitives}

\subsubsection{Motor planning}

Flash and Hogan (1985) developed a model for the generation of unconstrained coordinated multi-joint movements. They found that point-to-point movements result in approximately straight trajectories with bell-shaped tangential velocity profiles. Furthermore they discuss the reduction of tangential velocity when producing curved hand paths, and summarised this finding in the so-called twothird power law which allows a quantitative analysis of this pattern.

\subsubsection{Primitives}

In the field of motor control, primitives or elementary behaviours have a long tradition as building blocks of complex behavioural patterns. The idea can be traced back to studies of muscle synergies (cf. Finley et al., 1968). Already Arbib (1975) discusses their role and importance as elementary structures for the formation of complex actions in natural and artificial systems. The efficient combination of elementary constituents at the spinal cord level of the frog was shown by Mussa-Ivaldi et al. (1994). By stimulation they could show vector summation of force fields, that were subsequently discussed as the building blocks of 'a rich grammar of motor behaviours' (cf. Mussa-Ivaldi and Bizzi, 2000). Recent studies emphasise the advantages of a set of primitives for sensorimotor integration (see eg. Flash and Hochner, 2005) and imitation learning (Demiris and Hayes, 1999), and discuss concatenation strategies (cf. Breteler et al., 2003) as well as timing aspects at muscular level (cf. Pruszynski et al., 2010). A number of subsequent studies then tried to utilise these ideas in the field of robotics (cf. eg. Mataric, 2000).

\subsubsection{Error correction}

Archambault et al. (2011) studied the temporal evolution of motor intention, and the role of dorsal premotor and motor cortex in jumping-target reaches in monkeys. The found that after a target jump the plan of the movement to the initial target is smoothly changed into a plan for a movement heading towards the new target. Arguing on the basis of time lags in the neural activity they suggest that premotor cortex where the earliest correction signal could be 
found encodes commands from higher-order areas, while parietal regions are responsible for kinematics estimation.

The correction of errors in movement generation can be understood from two perspectives. One can assume that a constant tracking of movement precision on the basis of an internal model supports online corrections (cf. Gomi, 2008). Another idea which is supported by studies in monkeys (cf. Fishbach et al., 2005) suggests that no continuous control process but intermittent corrections are utilised by the central nervous system.

\subsubsection{Autopilot}

Pisella et al. (2000) studies movements towards a target jumped in a fraction of the trials. They found that first traces of the change towards the new target location could be found already about $150 \mathrm{~ms}$ after target jump, and it was rather hard to suppress the tendency to follow this change in location.

\subsection{Data processing and modelling}

\subsubsection{Trajectory-based data processing}

Hand position tracking at $90 \mathrm{~Hz}$ lead to relatively smooth trajectories, so that additional filtering was neither necessary nor desirable due to the tradeoff between data optimisation and filtering artifacts. Effects of 'invisible' highfrequency fluctuations during velocity approximation were prevented by choosing an appropriate approximation method. Although data was recorded in three-dimensional endeffector space, trajectory analysis was only conducted in the $\mathrm{x}-\mathrm{y}$ plane fronto-parallel to the participant, and parallel to the computer screen, because of the limited movement possibilities in z-direction (maximum 7.5 percent compared to freedom in $\mathrm{x}$ - and $\mathrm{y}$-direction).

\section{Velocity approximation with local noise reduction}

Endeffector velocity was approximated in a three-point estimation which uses the secant ${ }^{3}$ line through the points $\left(t_{i-d}, x_{i-d}\right)$ and $\left(t_{i+d}, x_{i+d}\right)$ :

$$
v_{i}=\frac{x_{i+d}-x_{i-d}}{t_{i+d}-t_{i-d}} .
$$

\footnotetext{
${ }^{3}$ It was based on the classical secant method $v(t)=\frac{x(t+h)-x(t-h)}{2 h}$ and lead to an approximate error of $E=\frac{-a(\tau)}{6} h^{2}$ for $t-h<=\tau<=t+h$.
} 

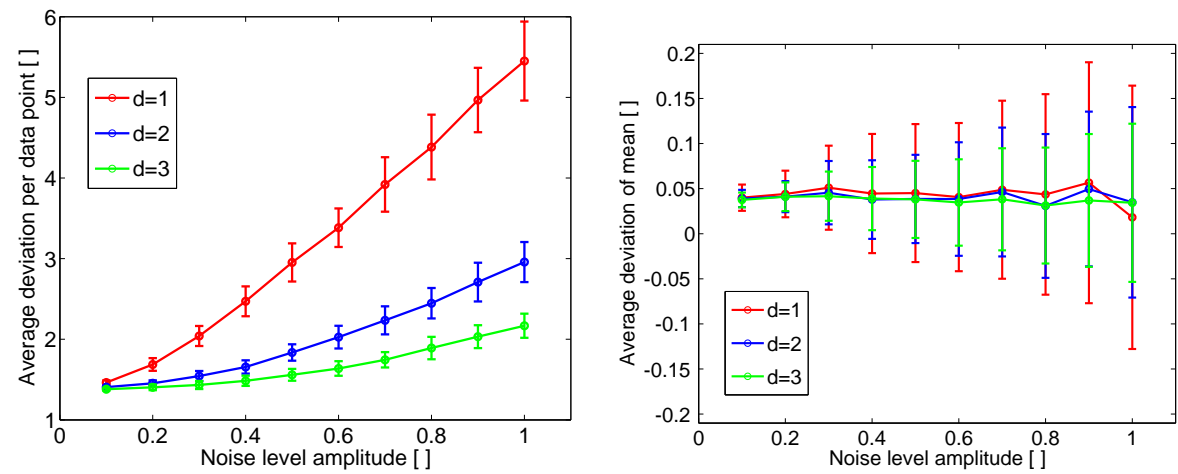

Figure 5.1: Performance of velocity approximation for three different step sizes $d$ in case of noisy sine wave (one period, 2 seconds, sampled at $10 \mathrm{~Hz}$ ), in dependence on noise amplitude (white noise). Averages are computed from 100 repetitions of 'measuring' the noisy system. Left: Average fitting error per data point compared to noise-free case. Right: Average difference in mean compared to noise-free case.

The advantage of this method is that upon appropriate choice of $d$ it produces rather reliable velocity estimates even when data is noise corrupted. Figure 5.1 shows the results of applying the algorithm to one period of a sine wave with a duration of 2 seconds which was sampled at $10 \mathrm{~Hz}$. Data was corrupted by additional white noise of different amplitudes. Ten data samples were generated at each noise level for velocity estimation with three different (sufficiently small) step sizes $d$. Estimation errors were summed over the whole period (compared to the noise-free case) and the difference in the mean (again compared to the noisefree case) was computed. Averages were computed over the samples. It can be seen that the method leads to an error reduction. For processing experimental data $d=3$ turned out to be a reliable choice.

\section{Kinks in the trajectory as traces of the replanning process}

When originally expecting target T2 in another location, and, hence, initiating a movement to the wrong direction, the participant was forced to replan in order to avoid repeating the trial. This results in a turnaround in the movement (cf. lower left or right subfigure of Figure 5.3) which is called 'kink' throughout this work.

Automated kink detection was implemented based on the geometric judgement criterion that would be applied by a human 'kink detector': There is a kink, if for the movement segment between target $\mathrm{T} 1$ and arrival at wall height 
there is an extremum (which does not only cover very few data points) in xdirection to the opposite side than where the movement finally heads.

\subsubsection{Bell-shaped velocity profiles and replanning}

The velocity trace of a straight point-to-point movement starting at $T_{0}$ with a duration of $T$ and a maximum velocity of $v_{m}$ was found to be bell-shaped (cf. Plamondon et al., 1993). I chose the minimum jerk related symmetric bell due to the fact that its higher derivatives remain zero at the tails causing smooth on- and offsets of the movements. The velocity profile can be written as

$$
v(t)=V\left(t-T_{0}\right)^{2}\left(t-\left(T_{0}+T\right)\right)^{2}
$$

(cf. Flash and Hogan (1985)) with a velocity factor $V=v_{m} /\left(\left(t-T_{0}\right)^{2}(t-\right.$ $\left.\left.\left(T_{0}+T\right)\right)^{2}\right)$. These 'bells' are considered to be connected to building blocks of behaviour (cf. Henis and Flash (1995)).

\section{Bell-shaped velocity profiles of correct direct movements towards tar- get T2}

A correction in movement direction (eg. first moving right, then moving left) can be thought of as an overlay of two velocity profiles according to $v=(1-\alpha) v_{1}+$ $v_{2}$. The first movement which started at time $T_{01}$ and would have lasted $T_{1}$ with a peak velocity of $v_{m 1}$ is stopped within $\alpha$ seconds after the onset $T_{02}$ of the second one (duration $T_{2}$, peak velocity $v_{m 2}$ ).

For this superposition of an incorrect movement with an according correction, I (least-square) fit Equation 5.2 to the data from correct movements to either side until the movement got decelerated. Deceleration takes place when approaching the curve around the obstacle. The detour to the wrong side never showed such a wide extent.

Before processing, for sake of simplicity the movement onset is determined using a velocity threshold of one percent of the peak velocity of the trial.

\section{Double bell-shaped velocity profiles}

To study timing and peak velocities of replanning processes the model from Equation 5.2 was extended to be applicable to the two opposing phases of a kink: the excursion to the wrong direction, and the correction. This was accomplished by overlaying two bell-shapes $v_{1}, v_{2}$ with maximum velocities of different signs 
$\left(\operatorname{sgn}\left(v_{m 1}\right)=-\operatorname{sgn}\left(v_{m 2}\right)\right)$ according to $v=(1-\alpha) v_{1}+v_{2}$ for the first $\alpha$ seconds ${ }^{4}$ after the onset of $v_{2}$ ). Only the x-component of the movement is considered.

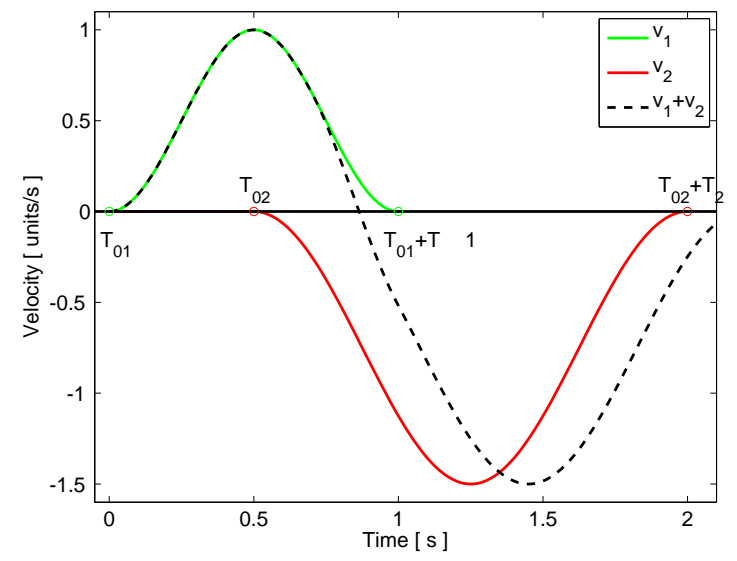

Figure 5.2: Overlapping velocity profiles. After the onset of $v_{2}$ at $T_{02} v_{1}$ fades away.

\section{Adaptive parameter determination for overlapping velocity profiles}

Given the x-velocity trace between the time of reaching target $\mathrm{T} 1$ and the time of reaching wall height in one trial $\left\{v_{t}, T_{T 1} \leq t \leq T_{W}\right\}$ the optimal parameters $T_{01}, T_{1}, v_{m 1}, T_{02}, T_{2}, v_{m 2}$ can be computed based on the following error function $E$. For sake of simplicity $v_{t}$ is written as $v(t)$ and $\hat{v}_{2}(t):=v_{2}(t)-v(t)$. Overlapping velocity profiles $\left(T_{01}+T_{1} \geq T_{02}\right.$, cf. left subfigure of Figure 5.2) the approximation error consists of five components:

$$
\begin{aligned}
E_{\text {over }}\left(T_{01}, T_{1}, v_{m 1}, T_{02}, T_{2}, v_{m 2}\right) & =\sum_{t<T_{01}} v(t)^{2}+\sum_{t=T_{01}}^{<T_{02}}\left(v_{1}(t)-v(t)\right)^{2} \\
& +\sum_{t=T_{02}}^{T_{02}+\alpha}\left(\left(1-\frac{t-T_{02}}{\alpha}\right) v_{1}(t)+\hat{v}_{2}(t)\right)^{2} \\
& +\sum_{t>T_{02}+\alpha}^{T_{02}+T_{2}}\left(\hat{v}_{2}(t)\right)^{2}+\sum_{t>T_{02}+T_{2}} v(t)^{2}
\end{aligned}
$$

Because of some parameters occurring as limits of summation, a mixed approach is chosen: $v_{m 1}$ and $v_{m 2}$ are computed by the gradient descent $v_{m i}^{n e w}=$ $v_{m i}^{\text {old }}=-\epsilon \frac{\delta E}{\delta v_{m i}}, i=1,2$, for appropriately chosen initial values and 'traditional'

\footnotetext{
${ }^{4}$ Typical values ranged around $0.6 \mathrm{~ms}$.
} 
cooling of $\epsilon$ (cf. Rosenblatt, 1958). The gradient descent for the remaining parameters is simulated by evaluating the according error functions for slightly altered parameters (e.g. in case of $T_{01}: E_{\text {over }}\left(T_{01}+\epsilon_{T}, T_{1}, v_{m 1}, T_{02}, T_{2}, v_{m 2}\right)$ and $\left.E_{\text {over }}\left(T_{01}-\epsilon_{T}, T_{1}, v_{m 1}, T_{02}, T_{2}, v_{m 2}\right)\right)$ and then taking the step into the best direction. $\epsilon_{T}$ again is cooled down over time in a way that guarantees convergence. In the implementation it has to be considered that a shift by $\epsilon_{T}$ can change the overlap state of $v_{1}$ and $v_{2}$.

Good initial values are provided by using the first two local extrema $E 1$ and $E 2$ of $v: v_{E 1}=v\left(T_{E 1}\right), v_{E 2}=v\left(T_{E 2}\right)$, and the root $v_{0}=v\left(t_{0}\right)^{5}$ such that $v_{m 1}=v_{E 1}, v_{m 2}=v_{E 2}, T_{01}=0, T_{1}=2 t_{E 1}, T_{02}=t_{0}$, and $T_{2}=t_{0}-2 t_{E 2}$.

$T_{01}$ can be seen as the time that the participant spends in T1 to get most information out of the system before deciding. $T_{02}$ can be considered as the time needed for replanning if this phenomenological approach of bell-shaped velocity profiles is valid. $\alpha$ indicates the time needed to fully abort the initial wrong movement.

This model provides deeper insights in the timing of what is going on when participants change their minds as a reaction to new information about the environment.

Extrema The assumption of the bell shape Equation 5.2 leads to a first derivative w.r.t $t$

$$
\dot{v}(t)=2\left(V\left(t-T_{0}\right)\left(t-\left(T_{0}+T\right)\right)\left(2 t-2 T_{0}-T\right)\right),
$$

which leads to the maximum $v_{\max }=\frac{V}{16} T^{4}$ at $t=\frac{T}{2}$.

\subsection{Results of trajectory-based analysis}

\subsubsection{General results on task performance}

Typical trajectories For each presented T2 location participants could produce two kinds of trajectories of valid movements: one heading "directly" towards $\mathrm{T} 2$ on the shortest way, or one first showing a movement into the wrong direction which has to be replanned and "substituted" by a correct one. Figure 5.3 shows typical examples of direct movements to the left and the right,

\footnotetext{
${ }^{5}$ It exists due to the theorem of Bolzano.
} 
as well as replanned ones. Furthermore, trajectories of movements heading towards $\mathrm{T} 2$ in the centre are depicted. Replanned movements towards the centre occurred only rarely in a few participants, so they are not separately discussed in the following.

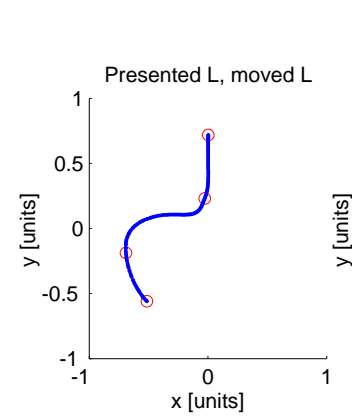

\section{Example trajectories}
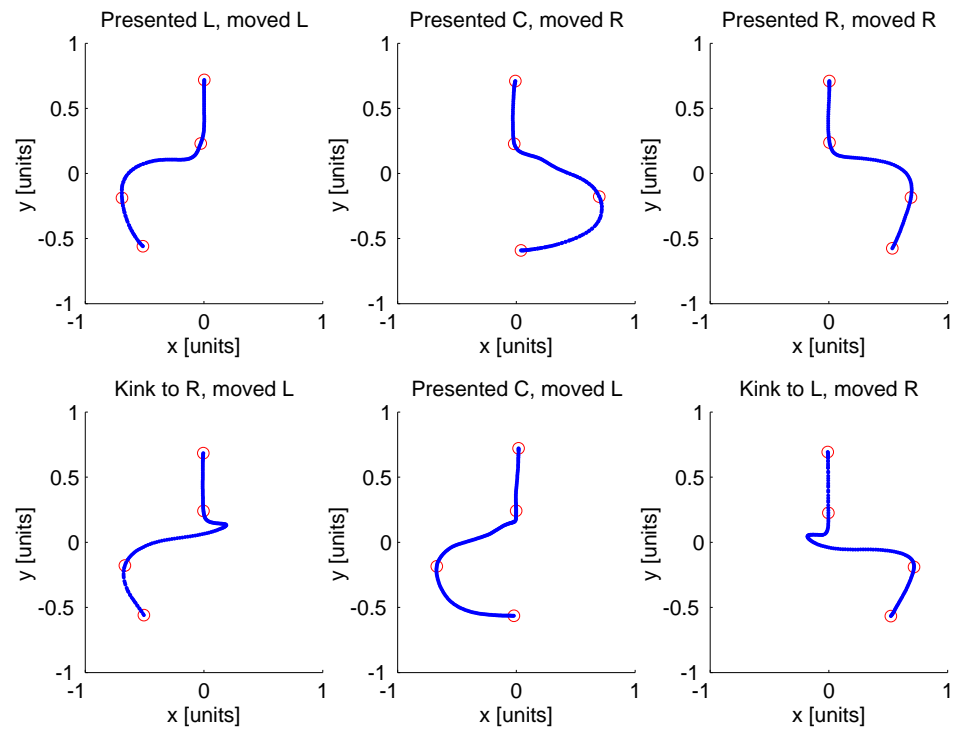

Figure 5.3: These example trajectories show important cases of trajectories that are created by the participants. Left column: Direct movement to the left, and movement with a "kink" towards the right before heading left. Centre column: Direct movements towards the central target, passing the wall on the right or the left side. Right column: Direct movement to the right, and movement with a "kink" towards the left before heading right. Note that the circles indicate the time of the according events, not target sizes.

Success statistics of producing valid trials It is not trivial that the participants manage to keep the time constraint around target $\mathrm{T} 1$ while still hitting the relatively small target. Table 5.1 summarises the number of necessary trials in order to produce the required amount of 250 valid trials ${ }^{6}$, and indicates that the posed task is indeed feasible. The imposed timing constraints only caused an additional work load of about five percent. No systematic differences concerning the influence of handedness or used hand could be found.

\footnotetext{
${ }^{6}$ Only data of the initR condition is displayed. For init $L$ the proportions were quite alike.
} 
Table 5.1: Number of trials that were needed to produces 250 valid ones, grouped by handedness and used hand. The dominant hand was used first in all participants. Data from one left-handed person using the right hand is missing.

\begin{tabular}{|l|c|c|c|c|}
\hline & Session 1 & Session 2 & Session 3 & Session 4 \\
\hline Right-handed, RIGHT & $285+/-9$ & $263+/-12$ & $259+/-4$ & $259+/-3$ \\
Right-handed, left & $277+/-14$ & $262+/-4$ & $263+/-9$ & $260+/-6$ \\
Left-handed, LEFT & $313+/-39$ & $273+/-9$ & $273+/-8$ & $270+/-7$ \\
Left-handed, right & $278+/-12$ & $263+/-4$ & $259+/-6$ & $260+/-7$ \\
\hline
\end{tabular}

Massive training leads to a decrease in trial completion time The average trial duration can be taken as a measure for task performance. For analysis each participant's movements of the same kind towards non-centre targets T2 were pooled for averaging. The final average of these mean trial durations is depicted in Figure 5.4(a) for the initL condition, and in Figure 5.4(b) for initR, respectively. The mild decrease in these completion times can be interpreted as the participants getting more and more used to the task. No significant ef-
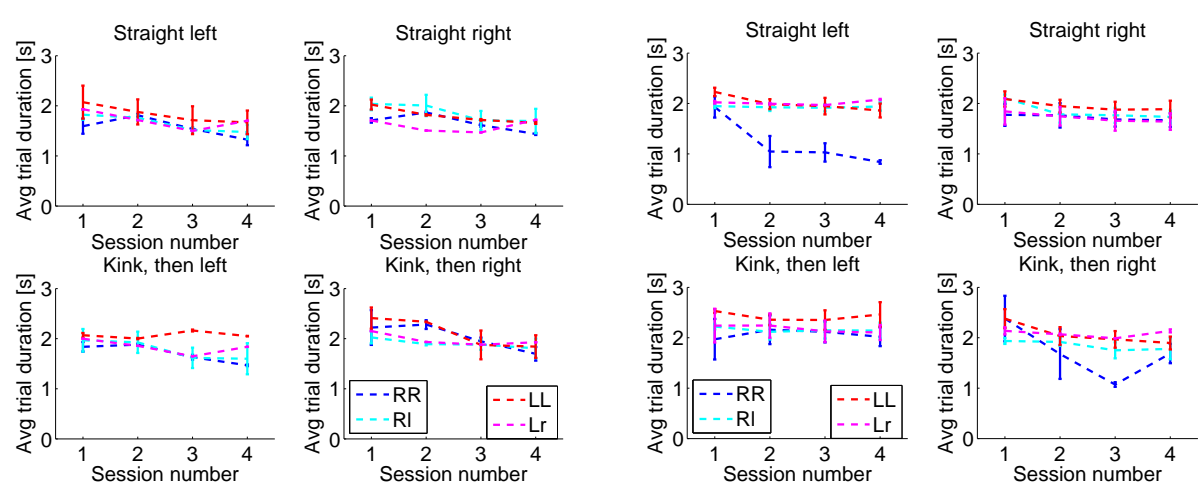

(a) Average trial completion times in condi-

(b) Average trial completion times in condition initL.

tion initR.

Figure 5.4: The averaged average duration of trials split by handedness and used hand (RR - right-handed using right hand, Rl - right-handed using left hand, LL - lefthanded using left hand, Lr - left-handed using right hand), as well as movement type (straight left/right; kink, then left/right) show a mild decrease in course of sessions.

fects of handedness and used hand can be found on overall trial duration. The eye-catching drop in completion time for right-handed subjects using their right hand in leftwards movements ${ }^{7}$ in the initR condition in Figure 5.4(b) stems from

\footnotetext{
${ }^{7}$ It can be seen for "straight left" as well as "kink, then right" movements.
} 
the fact that these kind of movements were rather seldom and only occurred in two of the fastest participants.

\subsubsection{Trajectory timing and replanning}

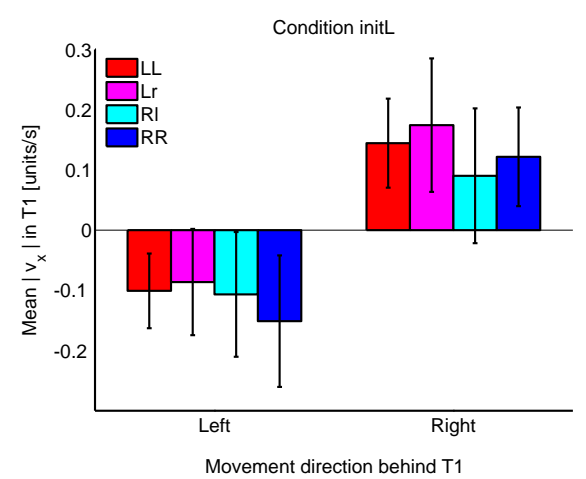

(a) Condition initL.

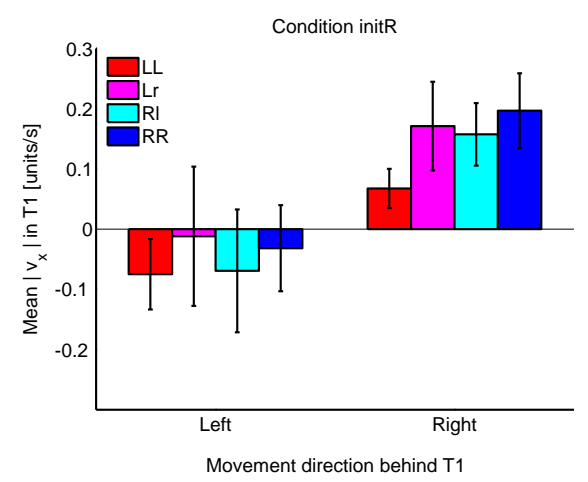

(b) Condition initR.

Figure 5.5: Average horizontal component of movement velocity in $\mathrm{T} 1$ across participants reflects choice of movement direction. Individual error bars show handedness and used hand conditions: LL - left-handed using the left hand, Lr - left-handed using the right hand, $\mathrm{Rl}$ - right-handed using the left hand, $\mathrm{RR}$ - right-handed using the right hand.

Strategy in target T1 The arrival in T1 was critical for further proceeding in the task, since the final movement target was revealed. Due to the time restriction in this area, the participants had to make up their mind before reaching T1. It turned out that the horizontal component of their hand velocity in $\mathrm{T} 1$ in each trial already reflected the choice of the upcoming movement (see averages of the session average of $v_{x}$ in Figure 5.5; velocity was estimated using the secant method from Equation 5.1 with a window size of $d=3$ ). Leftwards movements were preceded by negative horizontal velocity component, rightwards movements showed a positive one in $\mathrm{T} 1 .^{8}$

Participants were instructed to execute fast and smooth movements without any stops in T1. But the tradeoff between continuing the movement and keeping as much time as possible for processing the location of $\mathrm{T} 2$ resulted in individual strategies which can be characterised by the velocity of passing the intermediate target. The ones who went slower gave themselves more time to process the final

\footnotetext{
${ }^{8}$ The large variability between handedness and hand conditions for leftwards movements in the case of initR has to be traced back to its origin in the fact that this movement direction was chosen rather seldom.
} 
target location than those approaching T1 in a fast way (cf. Figures 5.9(a) and 5.9 (a) which illustrate the relation between the average velocity in $\mathrm{T} 1$ and the average delay there on an individual level). Figure 5.6 provides an analysis on the level of averages over single sessions of individual participants. The average delay in target $\mathrm{T} 1$ is the time difference between reaching this point and accelerating to a horizontal velocity $v_{x}$ larger than 0.012 units $/ \mathrm{s}(3 \mathrm{~mm} / \mathrm{s})^{9}$. On average it decreases, because the overall speed $v_{x}$ in either direction increases in both experimental conditions ( initL - Figure 5.6(a), initR - Figure 5.6(b)).

(cf. Figure 5.6).

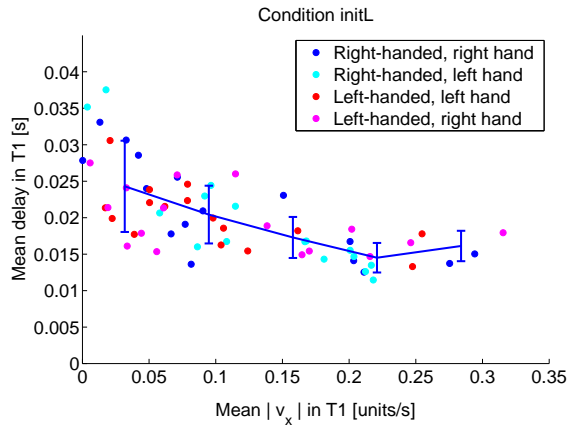

(a) Absolute velocity $v_{x}$ in $\mathrm{T} 1$ limits passage time, biased to the left.

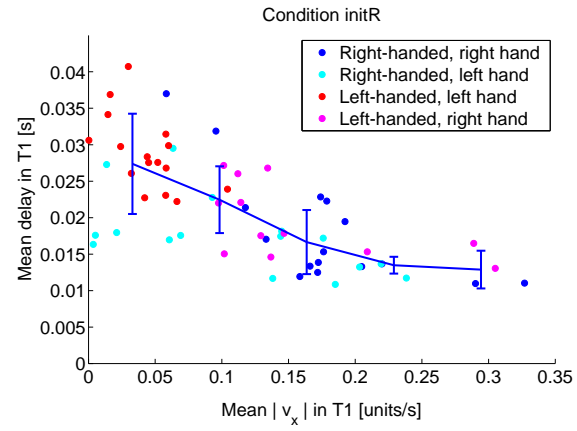

(b) Absolute velocity $v_{x}$ in $\mathrm{T} 1$ limits passage time, biased to the right.

Figure 5.6: Effects of passage strategy in intermediate target $\mathrm{T} 1$ on responsiveness to initial directional bias. Velocity determines time available for information accumulation. Circles indicate average absolute horizontal velocity and delay in T1 of single sessions of individual participants. The error bar indicates the trend calculated by averaging in 5 bins equally distributed between minimal and maximal velocity.

Peak velocities and their timing during obstacle avoidance The movement between the targets $\mathrm{T} 1$ and $\mathrm{T} 2$ reflects the individual choice of the participant in each trial. Traces of replanning - which are "turnarounds" with ensuing movements to the opposite side - or with respect to velocity, decelerations with ensuing acceleration of opposite sign - occur, if the participant's choice does not match presented T2 location. These two classes of movements - straight and replanned ones - are analysed in the following.

Since most interesting phenomena again appear in horizontal direction, I again limit the analysis to $v_{x}$. After the discussion of the onset of the directional excursion in $\mathrm{T} 1$ in the previous paragraph, now the other two main

${ }^{9}$ which is considered to be movement in Section 2.2.2 
characteristics - the timing and the magnitude of peak velocity - remain to be studied. These factors may reveal differences between the movements with the left and the right hand in participants of either handedness.

Figures 5.7(a) and 5.7(b) show the average timing of peak velocities of direct movements across participants for both experimental conditions. The upper row presents data for straight movements to the left or to the right. The lower row shows both the average timing of the velocity peak of the wrong excursion, as well as of the correcting part. Assessing roughly, handedness and used hand do not seem to play a consistent role. All estimates of mean horizontal velocity of the right-handed using the right hand (RR), the right-handed using the left hand (Rl), the left-handed using the left hand (LL), and the left-handed using the right hand are about the same. Since these average values for each session were calculated based on the averages of individual participants, important details might have been smeared out. For a more detailed comparison of the behaviour with the dominant hand and with the non-dominant one, Figures 5.7(c) and 5.7(d) show the average differences between peak timing using either hand. Averages were calculated from the average differences between the sessions of dominant and non-dominant hand in individual participants. Sessions which did not show movements to the direction of interest in one hand were excluded from calculation.

For straight movements in the condition initR (Figure 5.7(d)) it can be seen that right-handed individuals used to show a later velocity peak in the nondominant hand, compared to the dominant one (resulting in negative differences), while in the left-handed the opposite seemed to occur.

In the initL condition the peak timing of straight movements was more balanced between the dominant and the non-dominant hand in participants of either handedness. Only right-handed subjects showed an imbalance towards later peak velocities in the dominant hand compared to the non-dominant one for straight movements to the left in the first two sessions.

The timing of velocity peak of the wrong excursions to either side in participants of either handedness in both experimental conditions init $L$ and initR appears to be balanced. That means replanning leads to an "early" peak ${ }^{10}$ at about the same time, and suggests that the "replanning signal" is given at about the same time in all participants, independent of handedness and used hand. Note that the increased difference in timing of kinks to the left for left-handed

${ }^{10}$ compared to the peak time of the straight movement in the according direction 
participants in condition initR is likely to result from the imbalance in data due to the rare occurrence of kinks to the left in case of usage of the right hand.

Figures 5.8(a) and 5.8(b) depict the average peak velocities which on average are approximately the same for straight excursions to either side, as well as for corrected movements with a kink. This holds for both experimental conditions (initL, initR).

The differences between dominant and non-dominant hand in the peak velocities are slightly imbalanced between left- and right-handed participants. Positive differences in the right-handed participants show that they execute slightly faster movements with their (dominant) right hand. Negative differences in the left-handed ones show that they tended to execute movements with slightly higher peak velocities with their (non-dominant!) right hand. These effects cannot be consistently shown at acceptable levels of statistical acceptance, therefor further data would be needed for a final conclusion about its origin and significance.

Figures 5.9 and 5.10 provide an analysis of the relation of the absolute magnitude of horizontal velocity in $\mathrm{T} 1$ and the measures that were studied above on the level of global averages. For direct movements to either side a dependence of mean absolute peak velocity on the mean absolute horizontal velocity in target T1 can be seen (cf. Figure 5.9(a) for initL condition, Figure 5.9(b) for $i n i t R$ ). Interestingly, the relation does not show a continuing proportionality but saturates up at a mean absolute peak velocity of about 1.3 units/s. Also higher mean absolute peak velocities of excursions into the wrong direction and of subsequent correcting movements coincide with higher mean absolute horizontal velocity components (cf. Figure 5.9(c) for initL condition, Figure 5.9(d) for initR) and seem to saturate up at a certain level ( 0.8 units/s for excursions, 2.5 units/s for corrections). This suggests that these limits to peak velocities emerge from the interplay of the setup and the "universal" ${ }^{11}$ solutions the central nervous system makes use of - for example, motor primitives - which are invariant in their major characteristics across participants. In studies of human point-to-point movements these motor primitives are usually associated with a typical pattern of acceleration and deceleration that leads to bell-shaped velocity profiles (cf. Plamondon et al., 1993) which are symmetric in their simplest version. Higher peak velocities are not feasible in this context of self-paced movements. They would require too strong deceleration which would result in

\footnotetext{
11 "Universal" in this context may mean a pattern that occurs throughout different individuals.
} 
way higher forces resulting from movement dynamics. Hence, they would make the movement less "desirable" in terms of energy consumption.

Most interestingly, the timing of the peak velocities of the kinks in Figure 5.10(a) (for the condition initL) and Figure 5.10(b) (for the condition initR) remains constant independent of the velocity in $\mathrm{T} 1$ in both experimental conditions, and - since the latter also modulates the peak velocity - independent of the actual velocity of the wrong excursion. After on average $257 \mathrm{~ms}(0.255+/-$ $0.028 \mathrm{~ms}$ for initL, $0.260+/-0.028 \mathrm{~ms}$ for $i n i t R$ ) a deceleration can be observed in wrong hand excursions of all participants, no matter which handedness they have, which hand they use or under which experimental condition they executed the task. Furthermore, also the timing of the second peak (which stems from the correcting movement) in the horizontal component of endeffector velocity is independent off the velocity in the intermediate target.

Moreover, one can find a strong correlation between the average absolute horizontal peak velocity of the kinks and the average absolute horizontal peak velocity of the correcting movements (see Figure 5.11). The faster a participant executes the excursions into the wrong direction, the faster also the according corrections are.

Furthermore, the average trial completion time of replanned movements resembles the average trial completion time of the direct movements with an additional offset. If this offset is associated with the time until the replanning shows effects in the trajectory, a "regularity" in movement time can be stated.

Table 5.2: Reliability of the linear regression in Figures 5.11 and 5.12 in terms of t-values.

\begin{tabular}{|l|c|c|c|c|}
\hline \multirow{2}{*}{ Parameter } & \multicolumn{2}{|c|}{ Figure 5.11 } & \multicolumn{2}{c|}{ Figure 5.12 } \\
\cline { 2 - 5 } & initL & initR & initL & initR \\
\hline Slope & 24.239 & 21.991 & 18.501 & 19.766 \\
Intersection & 2.748 & 1.296 & 4.591 & 1.906 \\
\hline
\end{tabular}

Taken together with the above-mentioned results this strongly suggests a scheduling of the replanning process in the time domain. So this study lines up with a number of other studies that state separate planning of geometric and temporal aspects of movements (cf. Sosnik et al., 2004; Torres and Zipser, 2002, 2004). 


\subsubsection{Modelling of direct movements}

Single bell shapes were fitted to the direct movements heading left- and rightwards to the wall. Only the piece of the trajectory between target T1 and left or right passage of wall height was used. Averages for each session were computed split by handedness and used hand. Movement onset was defined as one percent of peak velocity (which corresponds to the definition of 'no movement' used in the stiffness section) and shifted to $t=0$ for sake of normalisation. The validity of this estimation during the deceleration towards the wall pass has to be taken cum grano salis since the trajectory is curved in this phase. Only the initial acceleration was needed later on for modelling the replanning process.

Figure 5.13 summarises the results. The lower left subfigure (left-handed using non-dominant hand) is only based on data from three instead of four participants. There were no significant differences between left- and rightwards movements within the groups. Movements were completed within about $800 \mathrm{~ms}$. Peak velocities were about 2 units per second. Movements with the dominant hand were slightly faster than the ones with the non-dominant hand, especially throughout the first sessions. Since this effect attenuated for later sessions it can be attributed to the neglected usage of this hand in movement tasks of everyday life (cf. Goble and Brown, 2008a).

\subsubsection{Replanning in terms of bell-shaped velocity profiles}

The bell-shaped approximations from the previous section were now used for modelling the correction of movement direction. The average profile of the direct movements to one side is chosen to be the erroneous choice that needs to be corrected. Figure 5.14 contains an overview of the average duration of the correcting movement (Figure 5.14(a)) and its peak velocity (Figure 5.14(b)) for all combinations of handedness and used hand in the initR condition. This suggests that timing and traces of replanning processes are independent of handedness and used hand.

Table 5.3 summarises the average replanning times until the information about the real location of target $\mathrm{T} 2$ is processed and in case of an erroneous choice of movement direction results in an endeffector acceleration towards the correct side. The results agree with the timing known from the 'auto-pilot' studies (see Pisella et al., 2000). They reported shortest latencies of about $120 \mathrm{~ms}$. 
Table 5.3: Average latency in seconds until correction could be found in the trajectory in terms of begin of acceleration towards the other side. Since the latency does not differ significantly for different directions of correction, all data from one session is treated at once (abbreviations: LL - left-handed using left hand, RR - right-handed using right hand, Lr - left-handed using right hand, $\mathrm{Rl}$ - right-handed using left hand).

\begin{tabular}{|c|c|c|c|c|}
\hline & Session 1 & Session 2 & Session 3 & Session 4 \\
\hline LL & $0.139 \pm 0.061$ & $0.140 \pm 0.070$ & $0.149 \pm 0.044$ & $0.169 \pm 0.056$ \\
RR & $0.135 \pm 0.043$ & $0.177 \pm 0.089$ & $0.159 \pm 0.133$ & $0.215 \pm 0.058$ \\
Lr & $0.140 \pm 0.036$ & $0.189 \pm 0.018$ & $0.202 \pm 0.069$ & $0.141 \pm 0.042$ \\
Rl & $0.167 \pm 0.037$ & $0.243 \pm 0.103$ & $0.187 \pm 0.043$ & $0.182 \pm 0.052$ \\
\hline
\end{tabular}

\subsubsection{On the question of symmetry of replanning process}

The very similar onset times (cf. Table 5.3), durations and peak velocities of movement corrections in left- and right-handed participants, as well as in participants of one handedness using different hands (cf. Figure 5.14) strongly suggest that the replanning process happens independently off handedness and used hand, and, hence, can be called symmetric with respect to them. 

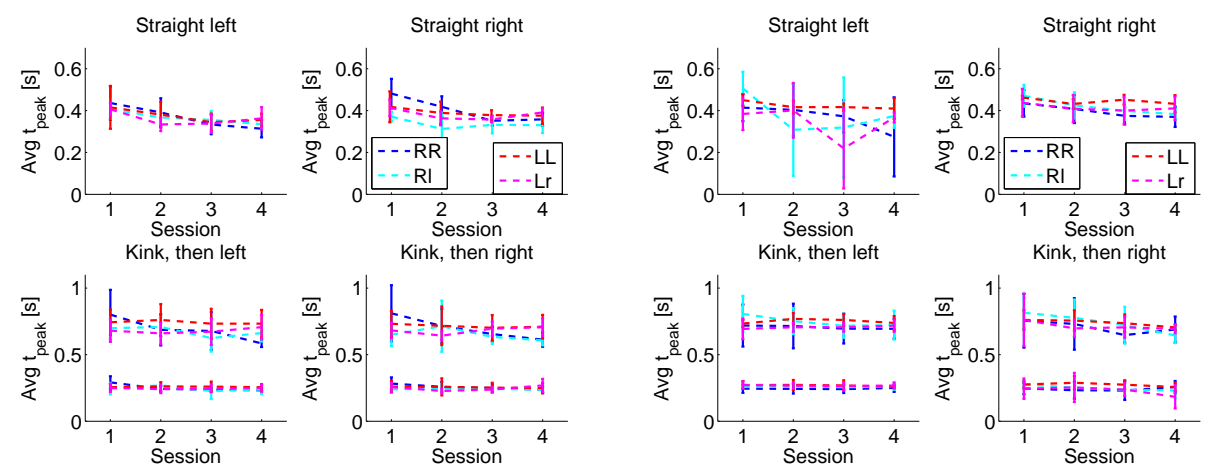

(a) Timing of peak velocities in condition initL. For replanned movements (lower row) the earlier peak belongs to the aborted excursion to the wrong side.

(b) Timing of peak velocities in condition initR. For replanned movements (lower row) the earlier peak belongs to the aborted excursion to the wrong side.
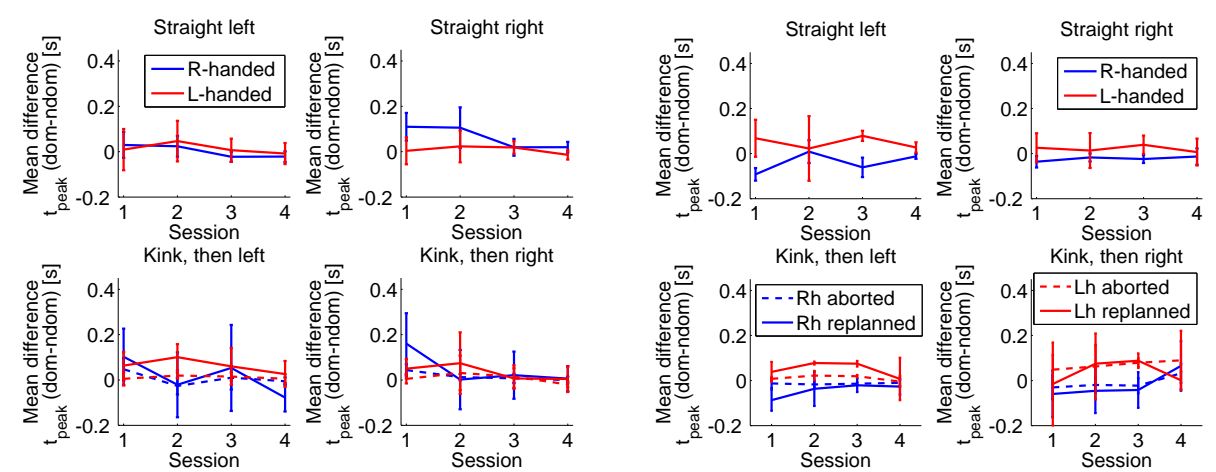

(c) Mean time difference of peak velocities between dominant and non-dominant hand in condition initL

(d) Mean time difference of peak velocities between dominant and non-dominant hand in condition initL

Figure 5.7: The averaged average timing of peak velocity by handedness and used hand, and according time differences between dominant and non-dominant hand for each handedness condition. Upper row of subfigures: Straight movements. Lower row of subfigures: Movements with kink. Average time of peak velocity of the kink as well as of the correcting movement are displayed. 

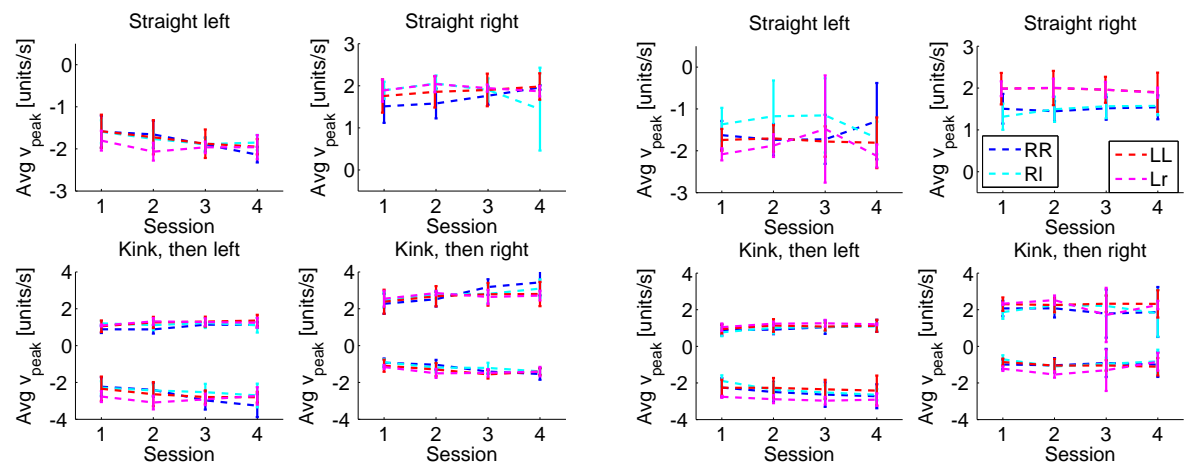

(a) Average horizontal peak velocities in condition initL. For replanned movements (lower row) the peak velocity with the lower absolute value belongs to the aborted excursion to the wrong side.
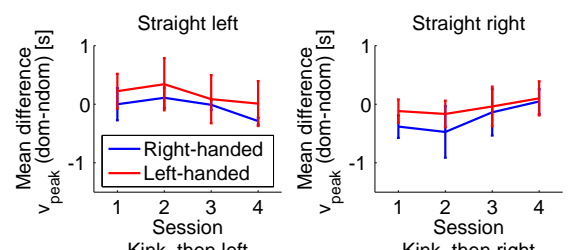

(b) Average horizontal peak velocities in condition initR. For replanned movements (lower row) the peak velocity with the lower absolute value belongs to the aborted excursion to the wrong side.
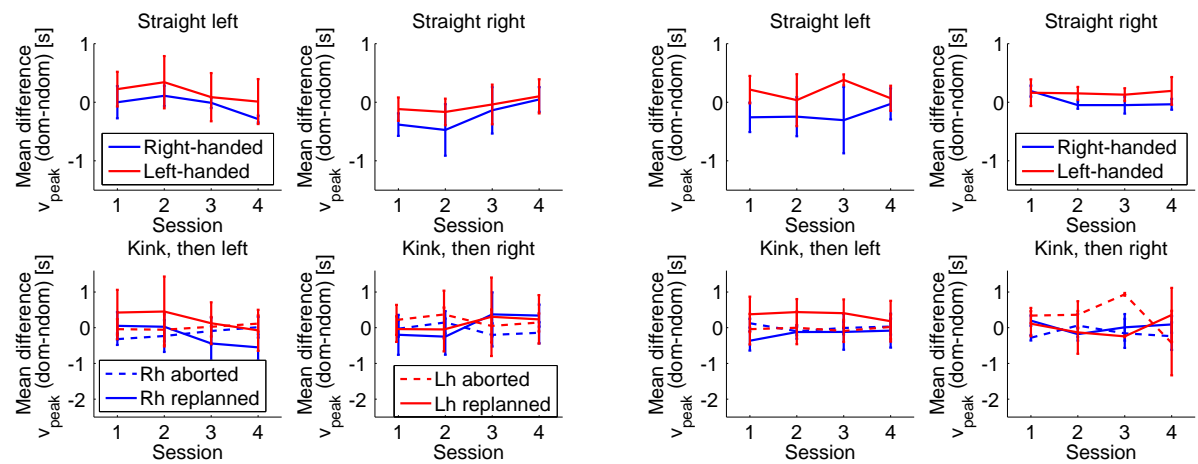

(c) Mean difference of horizontal peak velocities between dominant and non-dominant hand in condition initL

(d) Mean difference of horizontal peak velocities between dominant and non-dominant hand in condition initR

Figure 5.8: The averaged average horizontal peak velocities between $\mathrm{T} 1$ and wall height, split by handedness and used hand. Upper row of subfigures: Straight movements. Lower row of subfigures: Movements with kink. Average peak velocity of the kink as well as of the correcting movement are displayed. 


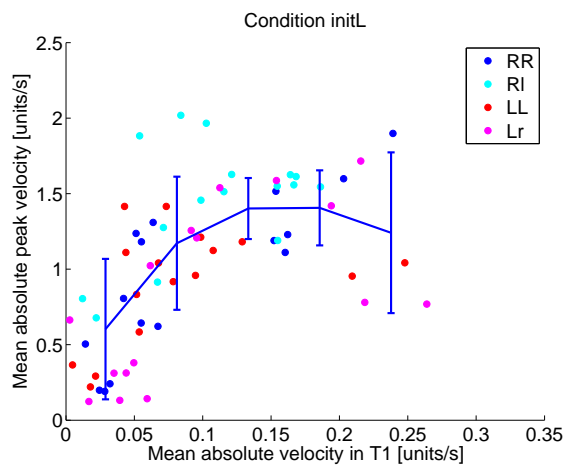

(a) Peak velocity of direct movements in dependence on velocity in T1. Condition initL.

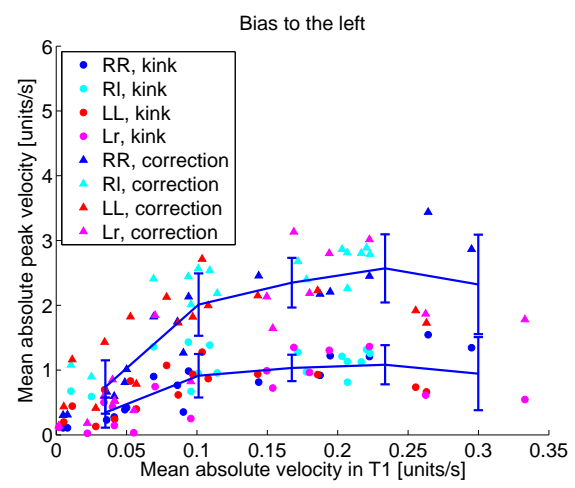

(c) Peak velocities of kinks and replanned movements in dependence on velocity in $\mathrm{T} 1$. Condition initL.

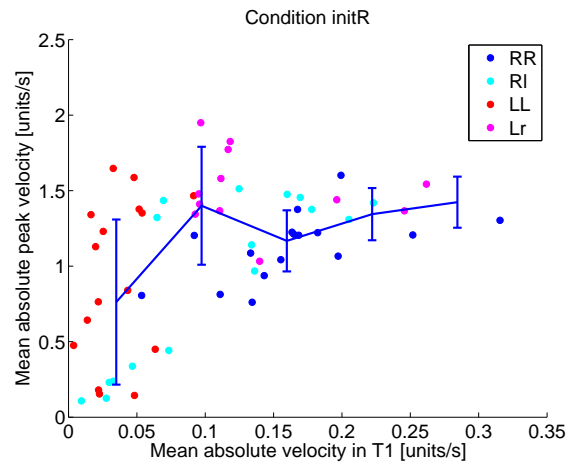

(b) Peak velocity of direct movements in dependence on velocity in $\mathrm{T} 1$. Condition initR.

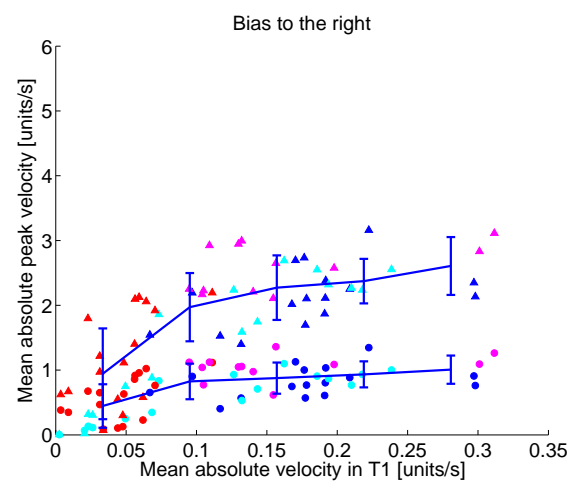

(d) Peak velocities of kinks and replanned movements in dependence on velocity in $\mathrm{T} 1$. Condition initR.

Figure 5.9: Effects of passage strategy in intermediate target $\mathrm{T} 1$ on overall movement performance. Velocity in T1 determines time available for information accumulation. Circles indicate average delay or velocity of single sessions of individual participants. The error bar shows the trend calculated by averaging in 5 bins equally distributed between minimal and maximal delay or velocity. 


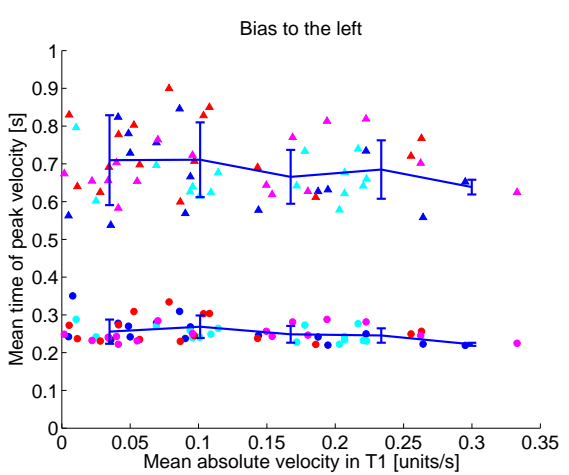

(a) Timing of peak velocities in kinks and replanned movements in dependence on velocity in T1. Condition initL.

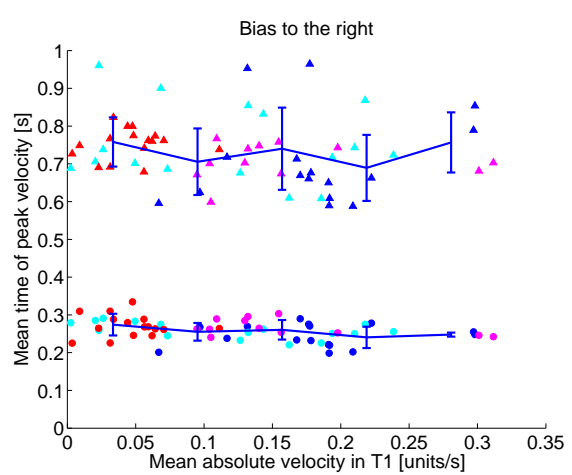

(b) Timing of peak velocities in kinks and replanned movements in dependence on velocity in T1. Condition initR.

Figure 5.10: Effects of passage strategy in intermediate target $\mathrm{T} 1$ on overall movement performance. Velocity in T1 determines time available for information accumulation. Circles indicate average delay or velocity of single sessions of individual participants. The error bar shows the trend calculated by averaging in 5 bins equally distributed between minimal and maximal delay or velocity.

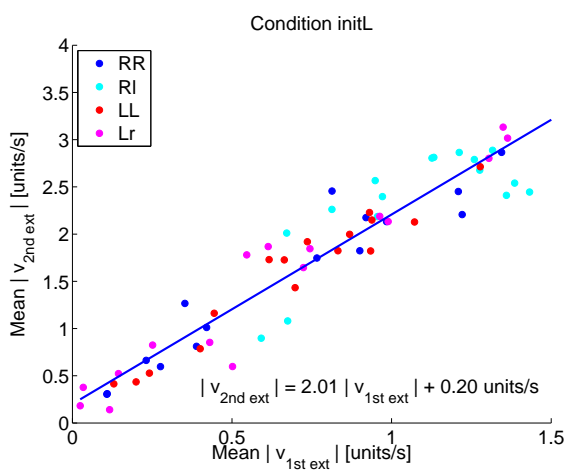

(a) Peak velocity of correcting movements in dependence on peak velocity of the kink. Condition initL.

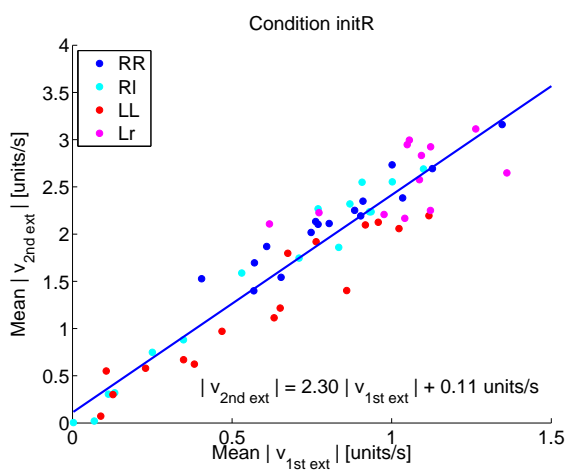

(b) Peak velocity of correcting movements in dependence on peak velocity of the kink. Condition initR.

Figure 5.11: Relation between first and second mean absolute peak velocities of corrected trials. First peak $\left|v_{1 s t ~ e x t}\right|$ corresponds to excursion to the wrong side, second peak $\left|v_{2 n d \text { ext }}\right|$ to correcting movement. Kinks to the left and the right are taken together for the session average. Dots indicate average velocities of single sessions of individual participants. The regression line is added for emphasis of the relation. 


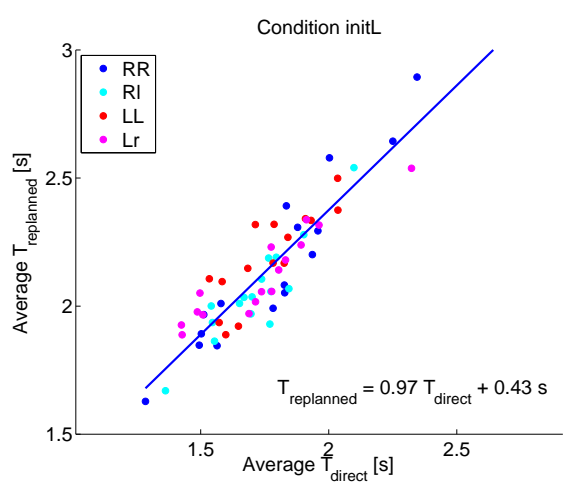

(a) Trial completion times in experimental condition initL.

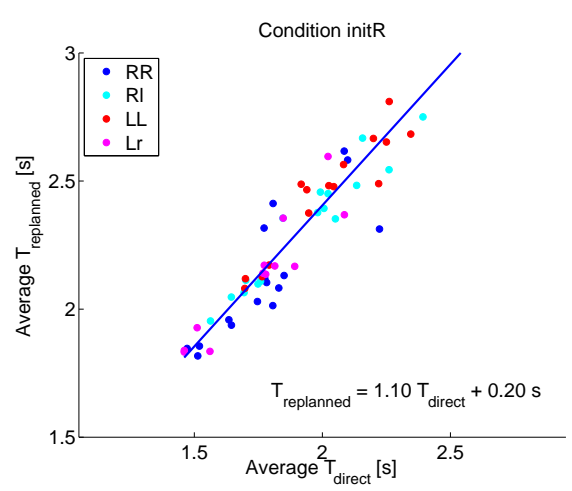

(b) Trial completion times in experimental condition initR.

Figure 5.12: Relation between average trial completion times of direct and replanned movements. Movements to the left and the right are taken together for the session average, kinks to the left and the right, respectively. Dots indicate average velocities of single sessions of individual participants. The regression line is added for emphasis of the relation.
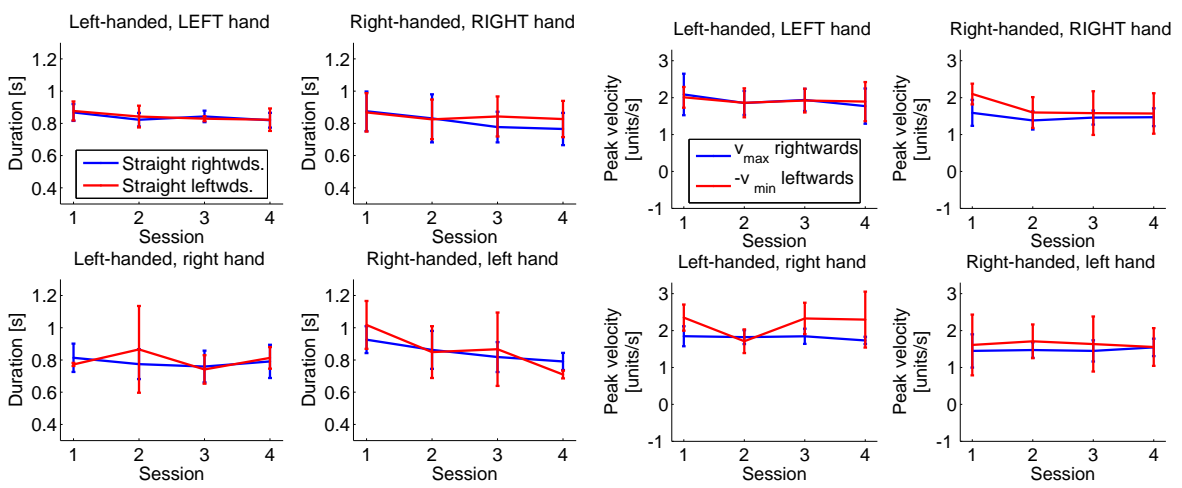

(a) Average duration of movement primitives.(b) Average peak velocity of movement primitives.

Figure 5.13: Descriptive modelling using bell-shaped velocity profiles: Average movement duration and peak velocity of direct movements (to the left (red) and to the right (blue)) for all sessions of all participants of the initR condition. Subplots are divided by handedness and used hand: upper row - session with dominant hand, lower row session with non-dominant hand. Left column - left-handed participants, right column - right-handed. 

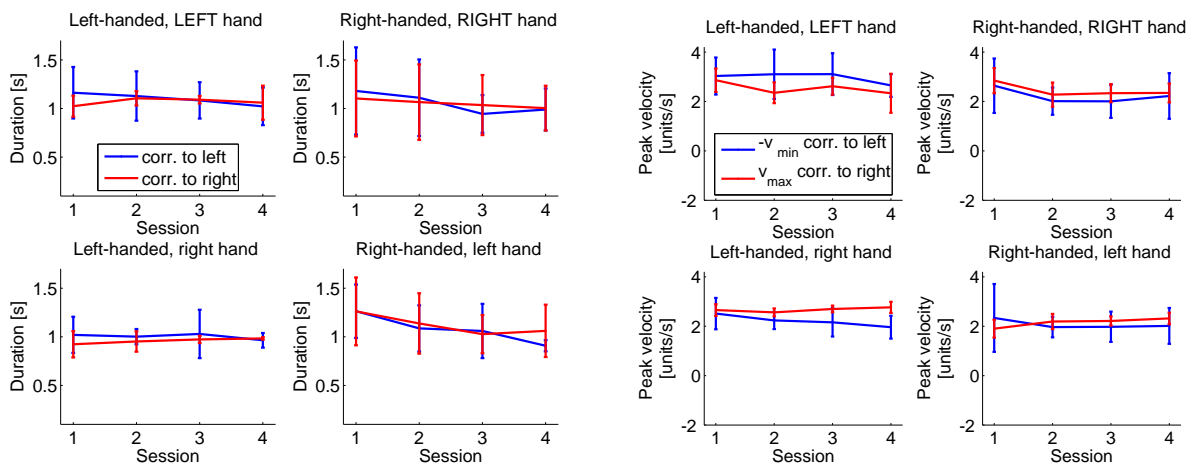

(a) Duration of correcting movements.

(b) Peak velocity of correcting movements.

Figure 5.14: Average movement duration and peak velocity of the correcting movement (to the left (red) and to the right (blue)) for all sessions of all participants of the initR condition. Subplots are divided by handedness and used hand. 


\subsection{Summary of trajectory-based analysis}

Major findings of this study are:

1. Although participants were instructed to move quickly and without a break in the intermediate target, the spectrum of individually employed strategies of dealing with the information bottleneck ranged from rather slow movements that provided as much time as possible for processing of the T2 location to fast movements that lead to frequent corrections.

2. Upper bounds to absolute peak velocities of each class of movements seem to emerge from the interplay between the setup and the motor control principles employed for solving the task. Since these limits are invariant across participants, also the employed principles, which may be motor primitives, seem to share main characteristics across participants.

3. While participants who are slower in the intermediate target also show lower peak velocities, faster participants only show peak velocities up to a certain threshold which seems to be imposed by the system.

4. Replanning processes in each hand are symmetric in timing and peak velocities when comparing corrections from left to right and right to left (cf. Section 5.4.5). Furthermore, the replanning process is symmetric in both hands of individual participants.

5. Moreover, the replanning process is also symmetric in participants of different handedness.

6. The timing of the movement corrections is equal across participants irrespective of handedness or used hand. The peak velocity of the excursion to the wrong side can be found at $0.257+/-0.028 \mathrm{~ms}$, the velocity peak of the correction at $0.712+/-0.094 \mathrm{~ms}$.

7. Furthermore, the average trial completion time remained invariant ${ }^{12}$ when comparing cases of direct movements to replanned ones. These timing issues provide further evidence for the separate planning of geometric and timing aspects of the movement.

\footnotetext{
${ }^{12}$ with a short processing delay
} 
8. The correction of erroneous choices of movement direction can be modelled sufficiently by only considering the horizontal component of endeffector velocity.

9. Replanning seems not to be affected by the spacial extend of the excursions or velocity magnitudes, but by the time needed for sensory processing and according reaction to the unexpected situation.

10. Modelling reveals latencies of the replanning process between 130 and 250 ms which match the "autopilot" threshold known from other studies (eg. Pisella et al., 2000). 


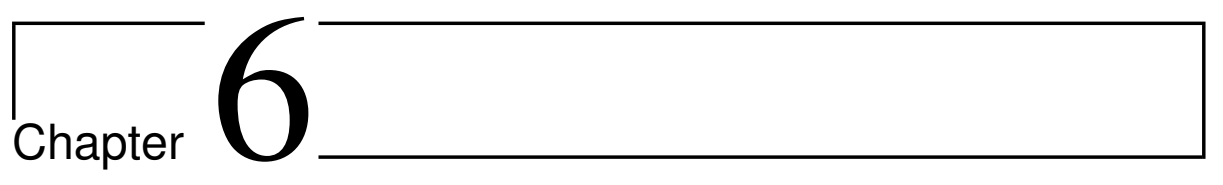

\section{Motor decisions under time}

\section{pressure}

\subsection{Introduction}

Reaching movements are governed by mainly unconscious estimates about the reliability of the own sensory system as well as about properties of the environment(cf. Koerding and Wolpert, 2006). In the following, the formation and adjustment of these estimates, more specifically the so-called prior, is examined (cf. Fiedler et al., 2010).

The study presented in this chapter aims at showing how humans handle a motor decision task under subjective time pressure, and at revealing the "strategy" that is applied to successfully cope with the task requirements of a twoalternative forced-choice setting. Knowledge about according regularities would be beneficial to robotics where often hardware constraints meet time constraints and limit the scope of applications. Heuristics inspired by nature could be of great importance in this context.

Furthermore, a better understanding of human motor decisions plays a key role in studying involved neural substrates during decision ${ }^{1}$ and error correction in case of erroneous choices. The results presented here base on the experiment described in Chapter 4 where the delay needed for visual processing serves

\footnotetext{
${ }^{1}$ See Cisek and Kalaska (2010) for a review of recent studies on the underlying neural mechanisms, and Deco and Rolls (2005) for a mathematical framework accounting for further effects, such as visual attention.
} 
as source of uncertainty. Although the necessary information for the choice was present at the time when the decision had to be made, the bottleneck of processing forces the participants to rely on their "general idea" about the distribution of target locations from previous trials.

Dependent on the experimental condition (initL or initR) the distribution of the final movement target was biased to one or the other side during the first tens of trials. By these means it could be shown that the formation of this "general idea" - or better: this prior - happened in dependence on previous decisions rather than on objectively presented data.

In contrast to purely cognitive decisions (such as choosing a letter from a set on the screen) motor decisions involve additional constraints related to properties of the motor system that have to be considered when it comes to the choice between different movements. The structured setup that is used for this study helps to disentangle some aspects of decision and planning interactions at the example of human upper limb movements by

- giving only two global movement alternatives (left or right),

- raising uncertainty by time critical presentation of information that is crucial for the decision making, and

- potentially making use of biophysically caused movement preferences.

On this basis I discuss the role of availability of information about the environment, and of additional motor-related constraints - such as handedness and used hand - as well as the role of attention. The reactions to wrong decisions manifest as excursion into the wrong direction, or - how these traces of replanning are called in the following - so-called "kinks" in the hand trajectory. These replanning reactions are studied with respect to certain aspects such as the timing of replanning and stereotypisation effects in movement choices which then lead to a discussion of motor primitives. The in depth understanding of the replanning process in terms of frequency of occurrence and causality can be used as the basis for follow-up studies which target at the brain regions that are involved in complex motor tasks.

A major distinction between the study presented in this chapter and classical motor decision studies is the relative time pressure which was imposed to the participants. Classical modelling using the reliability of sensor values eg. in a Bayesian approach will not be able to fully assess all information that is used for the decision. 
I show that with rising individual time pressure (rush in execution) more and more attention is drawn from perception towards the own behaviour which shows stereotypical patterns (see Section 6.4.3). For subsequent trials this is shown by statistical means in Section 6.4.1, for sequences up to a length of 30 trials using linear autoregressive modelling of the attention span in Section 6.4.2, and in general using a non-linear bifurcation model in Section 6.4.4. Furthermore, I show that the "default" choice of movement direction can be influenced by an initial directional overemphasis If this "bias" suggests a movement to the right, it is adopted by all fast participants independent of handedness and used hand (which are 85 percent of all sessions). The remaining slow ones show a balanced decision behaviour without strong preference of one or the other side. If the "bias" is in favour of the left, it only was matched by 60 percent of the fast participants. The other 40 percent of the fast subjects chose the right side for stereotypical repetition. The slow ones (25 percent of all sessions) again showed a balanced decision behaviour. Again handedness and used hand do not seem to be the major influencing variables.

\subsection{Motor decisions}

Motor decisions usually are studied in tasks where the individuals - be it animals, be it humans - have enough time to accumulate the needed information, and then can decide based on this material. Recent studies, for example, Westendorff et al. (2010) and Mattia et al. (2010) focus on the involvement of different brain areas during formation of the motor plan and its execution. Westendorff et al. (2010) studied the relative timing of motor-goal decisions in monkey dorsal premotor cortex and parietal reach region. During target presentation and movement execution a memory period of $800 \mathrm{~ms}$ up to $2 \mathrm{~s}$ was present up to $2 \mathrm{~s}$ was imposed. Mattia et al. (2010) developed an idea based on coordinated activity of neural populations in a theoretical model, and used it to study the temporal pattern of the involvement of different local neural populations in motor decision processes (in a countermanding task in nonhuman primates). In an fMRI study Soon et al. (2008) examined precursors of human motor decisions in a "self-paced" decision task where participants were shown a sequence of slides with letters at $2 \mathrm{~Hz}$ as "time indices". At any arbitrary time they could decide to press one of two buttons. The time of the awareness of their decision was matched with the slide that they perceived in this moment. 
Precursors of their decision could be found in their brain activity in prefrontal and parietal cortex up to 10 seconds before the decision came to awareness. Earliest predictive information was found in frontopolar and parietal cortex.

\subsubsection{Bayesian decision theory and accumulation of evi- dence}

Bayesian decision theory suggests how the central nervous system deals with the uncertainty that arises by noise in sensory signals and natural variability in motor outputs (cf. Koerding and Wolpert, 2006). Koerding and Wolpert (2006) suggest that these ambiguities are solved by means of Bayesian statistics by assigning probabilities to the according modalities and integrating the new information into a coherent image. That means, for the estimations of the position of one of our limbs the felt or seen location is combined with the previously experienced uncertainty of the involved sensory channels - which is also called prior. Decisions then are made rationally based on cost estimates which, for example, consider energy consumption of potential movements. The prior is constantly adjusted to provide a solid basis for the decisions no matter whether the situation is already known or a new movement is learnt.

Trommershaeuser (2009) stated that despite humans are bad in estimating the frequency of rare events in cognitive decision task, they perform well in equivalent motor decision tasks. After reformulating the problem in terms of Bayesian decision theory, she discussed resulting insights about how humans deal with uncertainty induced by noisy sensors during sensory-motor decisions.

\subsubsection{Handedness and decisions}

Not only energy consumption but also system preferences such as handedness can influence decision behaviour. Asymmetries in limb performance and related differences in sensory processing play an important role in the generation of motor behaviour (cf. Goble and Brown, 2008b). The dynamic dominance theory of handedness suggests that different sides are specialised in different aspects of motor tasks (trajectory control vs. postural control) (see Sainburg, 2002). Differences in sensory processing range from a longer time spent for visually monitoring the dominant hand in bimanual reaching tasks (cf. Honda, 1982) to different degrees of utilisation of proprioceptive feedback in the dominant 
vs. non-dominant hand (for further details see Goble and Brown, 2008b), and thereby also may influence motor decisions.

\subsection{Data processing and modelling}

\subsubsection{Event-based data processing}

Representation of presented target locations and initially chosen movement direction

For an analysis of preferences in movement direction, the 'originally chosen' movement direction behind target $\mathrm{T} 1$ is extracted as the direction of movement in the first $100 \mathrm{~ms}$ behind target T1. Further corrections are not considered here. On a trial by trial basis the series $\{x\}$ of chosen movement directions (also referred to as 'behaviour') is generated to code the movement direction behind T1:

$$
x_{i}=\left\{\begin{array}{ll}
-1, & \text { left } \\
0, & \text { invalid } \\
1, & \text { right }
\end{array} \quad s_{j}= \begin{cases}-1, & \text { left } \\
0, & \text { centre } \\
1, & \text { right }\end{cases}\right.
$$

The time series $\{s\}$ furthermore denotes the presented locations of target T2 behind the wall (also referred to as 'stimuli'). Note that $\{x\}$ and $\{s\}$ are longer than 250 trials since they also contain non-valid trials for further analysis. $\bar{x}=\sum x_{i}$ denotes the average behavioural preference of a whole session, $\bar{x}_{i}$ the average up to trial $i . \bar{s}$ and $\bar{s}_{j}$ are defined accordingly.

\section{Probability estimation based on frequencies}

Accumulative averaging To monitor the global trends in a time series $\{y\}$ an accumulative average (focusing on $y_{i}=1$ )

$$
P_{R}^{y}(t)=\frac{1}{t} \sum_{\tau=1}^{t} \delta\left(y_{\tau}-1\right)
$$

can be chosen for all trials $t . P_{R}^{s}$ is the relative frequency of presented locations of target T2 on the right side behind the obstacle (denoted by $R$ ). $P_{R}^{x}$ refers to the participants behaviour and reflects the fraction of movements to the right behind target T1. Due to the symmetry of the setup only one side of 
representation (and direction of movement) - the one encoded by 1 - needs to be considered in this computation. From Section 4.3.2 it is clear that $P_{R}^{s}(t)=0.45$. $P_{L}^{s}(t)=0.45$ and $P_{C}^{s}(t)=0.1$ for an according calculation. If on average the participant is able to keep track of the presented series, his choices cause $P_{R}^{x}$ to be in the expected range of $0.55+/-0.05$ dependent on the fraction of 'centre' presentations which also result in a rightwards movement.

On one hand, this averaging technique gives a good idea about the general tendency in what the participant is doing, but, on the other hand, it also smears out finer fluctuations in the directional choice towards higher trial numbers. To compensate for this drawback, a second averaging method is chosen to study the finer time course.

Accumulative averaging with limited window size To get a clearer idea of what is happening during the second half of the sessions, I chose to use a 'sliding average' that only uses data from a window of size $M$ from the previous trials for averaging

$$
P_{R}^{y}(t)=\frac{1}{M} \sum_{j=0}^{M} \delta\left(y_{t-j}-1\right)
$$

\subsubsection{Linear model of the decision process}

\section{Linear model of directional choices}

To study the influence of presented target locations and own behaviour of previous trials on the current behaviour a linear auto-regressive model with additional sensor input is been chosen. Consider the behaviour $x_{t}$ in trial $t$ as being influenced by the $p$ previous behaviours $x_{t-1}, \ldots, x_{t-p}$ as well as by the current sensor value $s_{t}$ and the $q$ previous ones $s_{t-1}, \ldots, s_{t-q}$.

$$
x_{t}=\sum_{i=1}^{p} a_{i} x_{t-i}+\sum_{j=0}^{q} b_{j} s_{t-j}
$$

When using this model with fixed values for $p$ and $q$ for a whole session, I refer to it as $\operatorname{ARMAX}(\mathrm{p}, \mathrm{q})$. When using it for smaller fractions $M$ of data points from a session, and then moving this window of evaluation through the data

to get an idea about the time course of the change in the coefficients, I call it sliding ARMA, or short SARMAX(p,q,M). 


\section{Parameter adaption}

For given $p$ and $q$ the coefficients $a_{i}, b_{j}$ can be found using least square fit based on a sufficient amount of data to average out local fluctuations.

\subsubsection{Nonlinear model of the directional choice}

Nonlinear model Since it is shown below (in Section 6.4.1) that $P_{R}^{x}$ does not approach to the expected range of $0.55+/-0.05$ on the long run I derive a nonlinear model that accounts for ongoing internal processes leading to this bias in choice. It may be called nonlinear dynamics model of prior formation.

The time course of the participant's prior reflected in the initial choice of movement direction shows two major features:

- a rather quick development towards a constant threshold during the session, and

- a slower adjustment of this threshold between the sessions and to some extend also during them.

It is shown in Section 6.4.1 that the directional tendency during the session is induced by a slight bias in the presented sequence of target $\mathrm{T} 2$ to the left or the right. If target $\mathrm{T} 2$ is slightly more often presented at the right side, then the participant develops the strategy to take rightwards movements as the default, i.e. $P_{R}^{x}(t) \rightarrow 1$ for many repetitions. Accordingly, $P_{R}^{x}(t) \rightarrow 0$ for a slight bias to the left. Both fixed points are stable, given a constant $P_{R}^{s}(t)$ on the long run. It is to assume that the presented sequence of target $\mathrm{T} 2$ locations is perfectly balanced at all time scales there will be no bias in the participant's choice of movement directions ${ }^{2}$. In the model this can be accomplished by an unstable fixed point at $P_{R}^{x}(t)=0.5$. For sake of simplicity the central presentation of target T2 in the catch trials is neglected in this model.

Let $P_{R}^{x}(t)$ be the participant's tendency to move to the right behind target $\mathrm{T} 1$ in the $t$-th trial. The simplest model

$$
P_{R}^{x}(t+1)=F\left[P_{R}^{x}(t)\right]
$$

with the above sketched features is a symmetric nonlinear dynamic with three fixed points (see Fig. 6.1). The dynamical evolution of $P_{R}^{x}(t)$ has to be at least

\footnotetext{
${ }^{2}$ But this still would have to be confirmed, since 'perfect balance' is practically not possible and possible approximations would result in a completely predictable switching of locations.
} 


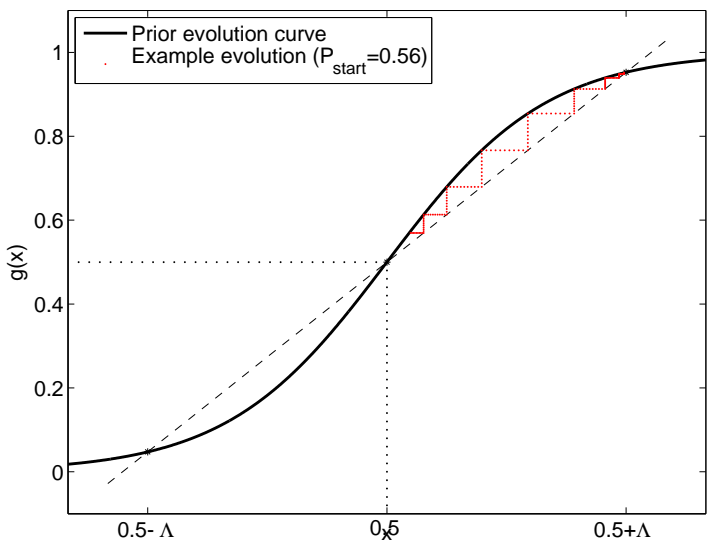

Figure 6.1: Nonlinear dynamics of prior evolution. Unstable fixed point at 0.5 , stable ones at $P_{-\infty}=0.5-\Lambda$ and $P_{\infty}=0.5+\Lambda$

a third-order polynomial of the structure

$$
g(x)=\alpha(x-0.5)-\beta(x-0.5)^{3}+0.5 .
$$

with $\alpha>1, \beta>0$, and for convenience $\alpha=1+\epsilon$. Since one needs $g(0.5)=0.5$, as well as $g(0)=0$ and $g(1)=1$ to resemble experimental data, $\alpha$ and $\beta$ depend on each other according to

$$
\frac{1}{2} \alpha-\frac{1}{8} \beta-\frac{1}{2}=0
$$

This finally means that

$$
\beta=4 \epsilon
$$

Some experimental evidence shows that the stable fixed points are not always exactly 1 , but slightly below. To account for this, let $P_{\infty}>0$, with $P_{\infty}=0.5+\Lambda$ (see Fig. 6.1). Since $g\left(P_{\infty}\right)=P_{\infty}$ Equation 6.6 leads to

$$
\alpha \Lambda-\beta \Lambda^{3}=\Lambda,
$$

what finally means that

$$
\beta=\frac{\alpha-1}{\Lambda^{2}}=\frac{\epsilon}{\Lambda^{2}} .
$$

For $P_{\infty}=1$ one gets back to Equation 6.8. 
It furthermore has to be ensured that the transform $g(x)$ does not drop after $P_{\infty}$. With Equation 6.6, and 6.10 one gets

$$
g(x)=(1+\epsilon)\left(x-\frac{1}{2}\right)-\frac{\epsilon}{\Lambda^{2}}\left(x-\frac{1}{2}\right)^{3}+\frac{1}{2} .
$$

and

$$
g^{\prime}(x)=(1+\epsilon)-\frac{3 \epsilon}{\Lambda^{2}}\left(x-\frac{1}{2}\right)^{2} .
$$

Thus, $g^{\prime}\left(x=P_{\infty}\right)=1-2 \epsilon$ which is greater than 0 for $\epsilon<0.5$ what is true for all cases.

For the practical evaluation of the model, $\epsilon$ can be assessed using

$$
\ln (1+\epsilon)=\frac{1}{\tau}
$$

with $\tau$ being the time constant of where the prior does not change significantly anymore (cf. Nonlinear Dynamics textbooks).

Parameter estimation of nonlinear autoregressive model of the prior Instead of manually tuning parameters after graph inspection the best $\epsilon, \Lambda$ and $P_{\text {start }}$ can be found using a simple algorithm that minimises the prediction error

$$
E=\sum_{t=1}^{N}\left|P_{r}(t)-P_{r}^{\text {model }}(t)\right|
$$

for given search intervals and search step size for each of the parameters. Each session is treated like this.

\subsection{Results of event-based modelling}

\subsubsection{Stereotypical decision patterns}

Bias in terms of kink counts The participants' chosen direction of movement was detected behind target $\mathrm{T} 1$ where they either moved to the right to catch T2 at the right behind the wall, or to the left, respectively. Given 250 trials of which 112 to 114 were dedicated to each side, the directional preference can be narrowed down to the error in the guess of movement direction. Tables A.1 (condition: initR) and A.2 (condition: initL) in the appendix give an overview of these errors which are referred to as 'kinks' due to their shape in 
Table 6.1: Coarse classification of responsiveness to an initially overrepresentation of one T2 location (initL - left, initR - right). Type pro-bias means preference of that direction, anti-bias preference of the opposite, mixed indicates a mix of both preferences in different sessions, and no bias stands for a balanced behaviour. (One left-handed participant in initL condition in non-dominant hand is missing.)

\begin{tabular}{|c|c|c|c|c|}
\hline \multirow{3}{*}{ Overall behaviour } & \multicolumn{4}{|c|}{ initL } \\
\hline & \multicolumn{2}{|c|}{ Left-handed } & \multicolumn{2}{|c|}{ Right-handed } \\
\hline & $\begin{array}{l}\text { dom. } \\
\text { hand }\end{array}$ & $\begin{array}{l}\text { ndom. } \\
\text { hand }\end{array}$ & $\begin{array}{l}\text { dom. } \\
\text { hand }\end{array}$ & $\begin{array}{l}\text { ndom. } \\
\text { hand }\end{array}$ \\
\hline pro-bias & 1 & 1 & 3 & 2 \\
\hline anti-bias & 1 & 2 & 1 & 2 \\
\hline mixed & 1 & 1 & - & - \\
\hline no bias & 1 & 0 & - & - \\
\hline $\begin{array}{l}\text { total number } \\
\text { of subjects }\end{array}$ & 4 & 4 & 4 & 4 \\
\hline \multirow{3}{*}{ Overall behaviour } & \multicolumn{4}{|c|}{ initR } \\
\hline & \multicolumn{2}{|c|}{ Left-handed } & \multicolumn{2}{|c|}{ Right-handed } \\
\hline & $\begin{array}{l}\text { dom. } \\
\text { hand }\end{array}$ & $\begin{array}{l}\text { ndom. } \\
\text { hand }\end{array}$ & $\begin{array}{l}\text { dom. } \\
\text { hand }\end{array}$ & $\begin{array}{l}\text { ndom. } \\
\text { hand }\end{array}$ \\
\hline pro-bias & 2 & 3 & 4 & 3 \\
\hline anti-bias & - & - & - & - \\
\hline mixed & 2 & - & - & - \\
\hline no bias & - & - & - & 1 \\
\hline $\begin{array}{l}\text { total number } \\
\text { of subjects }\end{array}$ & 4 & 3 & 4 & 4 \\
\hline
\end{tabular}


the trajectory ${ }^{3}$. Table 6.1 summarises the results using a coarse classification of behaviour in terms of responding to the initially overrepresented direction in all session (pro-bias), preferring the opposite direction in all sessions (anti-bias), showing balanced choices throughout sessions (no bias), and different levels of response to the overemphasised direction ranging from pro-bias to anti-bias in different sessions (mixed).

Given initR, we can see that almost all participant - no matter whether rightor left-handed - using either hand preferred the direction which was overemphasised during the first 50 trials. There was noone who preferred movements to the opposite side. The ones who did not show this suggestibility remained with an approximately balanced number of choices of movements to the left and to the right.

In initL, the situation gets more complicated, and differences between subjects of different handedness became apparent. All right-handed participants got "biased" to prefer movements in one direction. Three out of four chose the initially overrepresented direction using the dominant hand. Two out of four chose this direction using the non-dominant hand. Whereas from the lefthanded subjects only one out of four chose to pick this direction when using the dominant hand, and two other participants when using their non-dominant hand. Furthermore, one participant did not get biased at all in any session in the dominant hand, and again another subject showed the full range of getting biased - from no bias in the first session over bias in one of the directions, then again showing no bias, and finally a preference of the opposite direction.

In the Section 6.4.3 I come up with a discussion of this phenomenon in the light of different amounts of attention that is drawn to visual versus kinesthetic modalities.

Connection between movement speed and kink counts The absolute horizontal velocity $\left|v_{x}\right|$ in target $\mathrm{T} 1$ has been shown to be related to the average performance in individual trials. Figure 6.2 now gives a first idea about the connection of the (average) local measure and the global kink count measure. While slower movements result in a broader range of erroneous directional choices, higher velocities typically entail a higher number of errors.

Connection between movement speed and responsiveness to directional "suggestions" Furthermore, the individual behaviour when passing

${ }^{3} \mathrm{~A}$ further working definition of the terminus was given in Section 5.3.1 


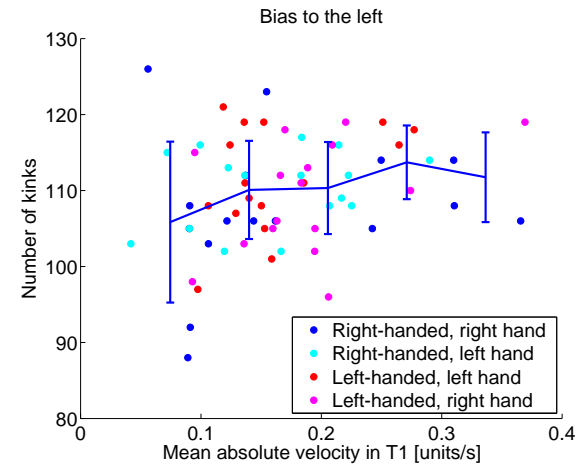

(a) Velocity in T1 affects number of errors in movement direction, biased to the left.

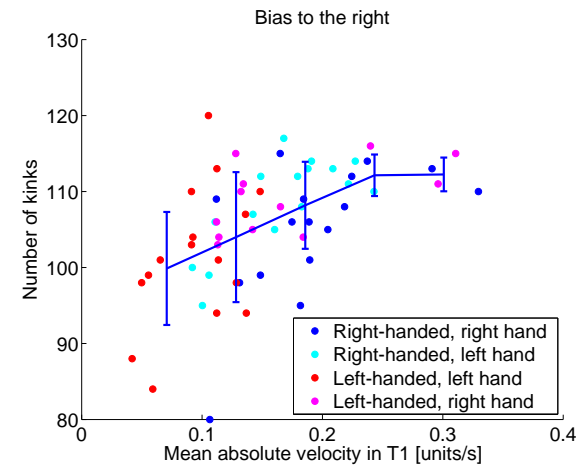

(b) Velocity in T1 affects number of errors in movement direction, biased to the right.

Figure 6.2: Effects of passage strategy in intermediate target $\mathrm{T} 1$ on number of movements that have to be corrected in direction. Velocity determines time available for information accumulation. Circles indicate average velocity of single sessions of individual participants. The error bar shows the trend calculated by averaging in 5 bins equally distributed between minimal and maximal velocity.

the intermediate target strongly influences the overall responsiveness to the bias. Figure 6.3(a) and 6.3(b) show relation between the average horizontal velocity $v_{x}$ in $\mathrm{T} 1$ and the overall probability of choosing a movement to the right in the end of a session. Individual dots indicate single sessions of single participants. From the average horizontal velocity of a session it is possible to predict the average preference of the movement direction.

Local statistics and global behaviour A look on the local statistics of the time series of each session, and the differences between them revealed a first idea why participants get biased. Figure A.1 shows how the previously shown final target location of the previous trial influences the choice of movement direction in the next step. For right-handed subjects either being biased to the left or the right, we can already see the tendency to prefer the direction of the bias from the first session on, no matter which location of target T2 had been shown in the previous trial. This holds for data from the dominant hand as well as from the non-dominant hand. Preference of the biased direction further increased throughout sessions. In left-handed participants using their dominant hand the initial susceptibility the bias was less pronounced. In the 'right bias' condition a similar increase in preference of the biased direction can be found. In the 'left bias' condition up to now only data from two participants using the dominant 


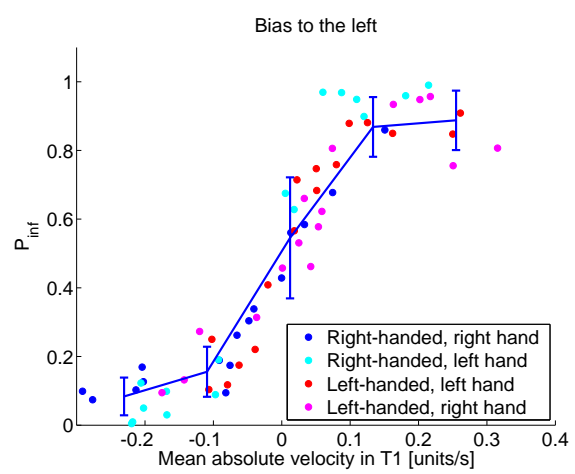

(a) Velocity in T1 modulates $P_{\infty}$, biased to the left.

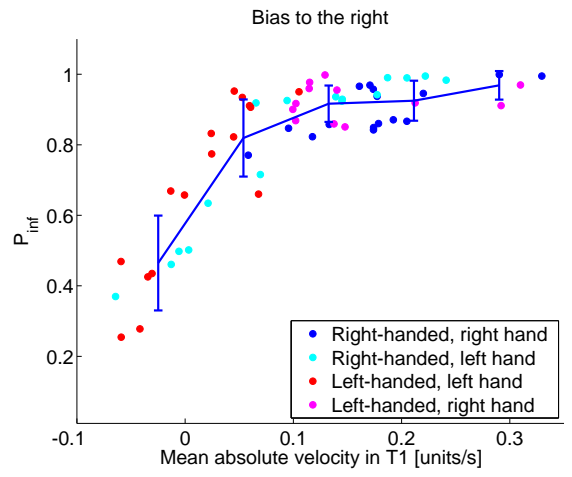

(b) Velocity in T1 modulates $P_{\infty}$, biased to the right.

Figure 6.3: Effects of passage strategy in intermediate target $\mathrm{T} 1$ on responsiveness to initial directional bias. Velocity determines time available for information accumulation. Circles indicate average delay or velocity of single sessions of individual participants. The error bar shows the trend calculated by averaging in 5 bins equally distributed between minimal and maximal delay or velocity.

hand, and one using the non-dominant hand is available, so it is hard to tell whether the attenuation of the directional preference is systematic or just a side effect of the small number of participants. Figure 6.4 further summarises Figure A.1 by highlighting the influence of previously shown T2 location on the preference to move towards or opposite to the initially overrepresented direction. A coincidence of all graphs from all presentation conditions means that the choice is independent of the presented target location.

Figure 6.5 summarises Figure A.2 from the Appendix, and provides insight on the question whether participants just tend to chose the same movement direction as in the previous trial. One might get this suspicion since it would serve as a simple "explanation" for the setup using an initial overrepresentation of one direction. Each graph in Figure 6.5 shows the difference between the probability to chose the same movement direction again and to do chose the opposite one. Thus, values around zero indicate "balance" between doing the same thing again and doing the opposite. Positive values stand for the tendency to repeat the same movement again and again, negative values indicate the inclination to try a movement to the other side in the next trial. Note that "same" and "opposite" always refers to the previously chosen movement direction (red graph: left, green graph: right). So, positive values in the green graph indicate a preferred choice of movements to the right, negative values in the green indi- 

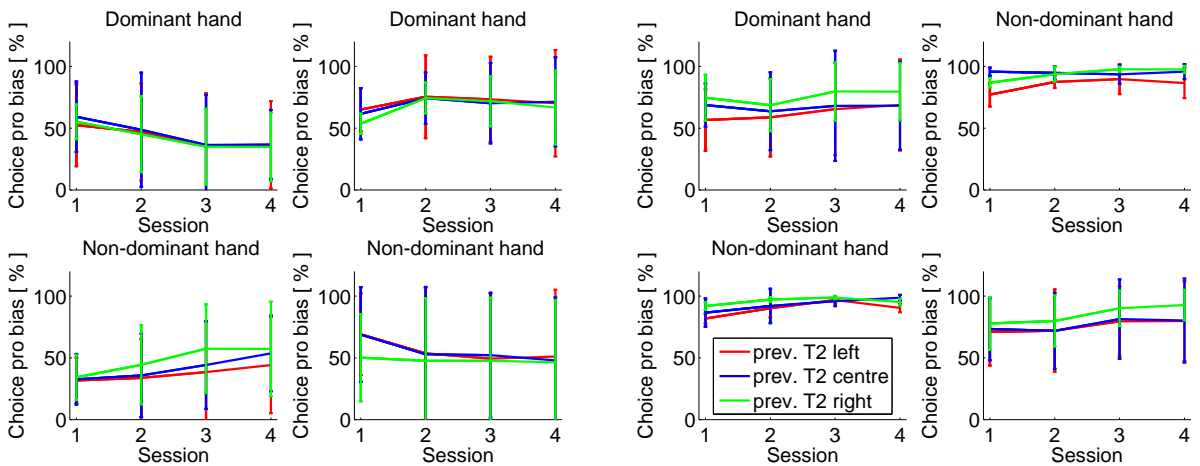

(a) Condition initL.

(b) Condition initR.

Figure 6.4: Consistency of directional choices in subsequent trials evaluated in relation to the "biased" direction. Difference of pro- and anti-"bias" choices of movement direction. In subfigures: Different graphs show different conditions of previously shown target T2 location. Data is split by handedness and used hand: The left column contains data for left-handed participants, right one for right-handed, respectively.

cate the same. Hence, symmetry with respect to the abscissa between the two graphs shows a strong preference of one direction. Coincidence of both graphs indicates the same underlying strategy for both directions of movements.

In the condition initR (Figure 6.5(b)) a clear preference to repeat rightwards movements can be read from the positive values in the green graphs and the negative ones in the red ones for right-handed participants using both hands, as well as left-handed participants using the non-dominant hand. An exception are the left-handed subjects using their dominant hand. Throughout all sessions they showed balanced choices of same and opposite movement direction, given a movement to the left in the previous trial. This suggests that there is a weak contribution of handedness.

Figure A.2 illustrates the dependence of directional choice on previously preferred movement direction. It raises further ideas about the tendencies found in Figure A.1, which dealt with sensory data.

For studying possible reasons of the bias in kink counts in dependence on bias in the presented series of target T2 locations and on other parameters such as handedness and used hand the limit probability $P_{\infty}^{x}$ is calculated. It not only comprises kinks but also direct movements to the right, and cases of a central target T2 in which a rightwards movement is chosen. Dotted graphs in Figures 6.15 and 6.16 show $P_{\infty}^{x}$ for each participant of the biased-to-the-right condition. (Figures 6.17 and 6.18 show the results for bias to the left.) The upper row 

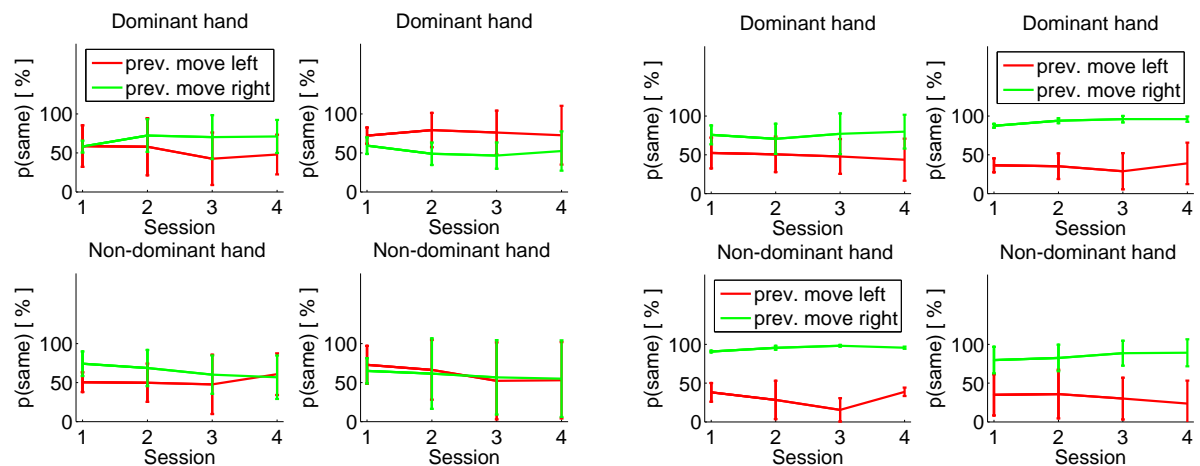

(a) Condition initL.
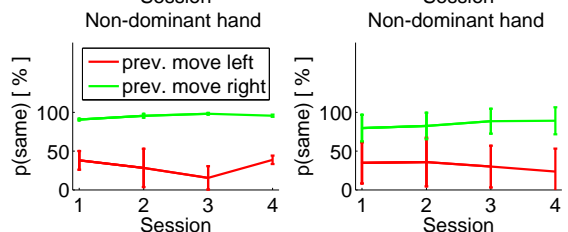

(b) Condition initR.

Figure 6.5: Consistency of directional choices in subsequent trials evaluated in relation to the "biased" direction. Difference of pro- and anti-" bias" choices of movement direction. Different graphs show different conditions of cased of previously chosen movement direction. Data is split by handedness and used hand: The left column contains data for left-handed participants, right one for right-handed, respectively.

contains data from the dominant hand, the lower from the non-dominant one. In most participants $P_{\infty}^{x}$ saturates up to a value significantly distinct from the expected range of $0.5+/-0.05$. Major exceptions are the right-handed KF using the left hand and the left-handed MT using the left hand (who did not converge to one decision pattern but changes them throughout sessions). Furthermore, there are tendencies to stay with $P_{\infty}^{x}$ close to $0.5+/-0.05$ in the first session of the right-handed CK using the left hand, the beginning of first session of the right-handed DB using the right hand, and in the first two sessions of the left-handed SA using the left hand. This suggests that hand usage may play a role. But it does not look like the only influencing parameter.

\subsubsection{Linear model of attention span}

Error pattern from linear modelling The usage of the ARMA model with different window sizes can be understood as a local examination of (maybe subconscious) attention windows in the presented sequence of target locations as well as in the own decision behaviour. The resulting error pattern provides - similar to recurrence plots (Eckmann et al., 1987) - 'hidden' information about the two time series. Figure 6.6 summarises two specific cases of decision strategies. Figure 6.6(a) depicts the ARMA error pattern for artificial data with an underlying oscillating choice of movement directions, that repeats every 15 trials. 


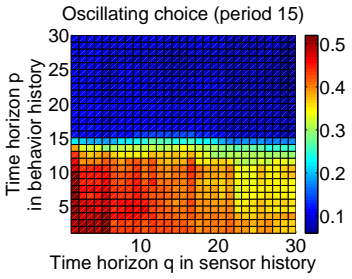

(a) ARMA modelling errors of time series with underlying oscillating movement strategy (doing the same after 15 repetitions).

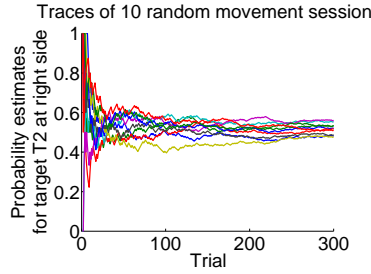

(b) Traces of the estimated probability to move to the right from ten randomly generated sequences of movement direction.

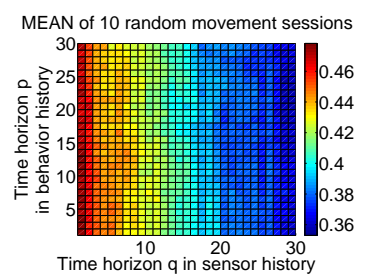

(c) Average ARMA modelling errors of ten randomly generated sequences of movement direction.

Figure 6.6: Illustration of the average "decision behaviour" of oscillating or random choices of movement direction in terms of estimated prior and ARMA error pattern.

This period is visible in the error cutoff at a behavioural window size of about 15. Figure 6.6(c) shows the resulting average error pattern of ten randomly generated sequences of left- and rightwards movements. This strategy results in a bar of high error aligned with the behavioural axis. Further perceivable simple strategies ${ }^{4}$ can be discussed as limit cases of the above discussed strategies: The alternating choice of left and right in subsequent trials would result in an oscillation with period 2, a constant choice of only rightwards movements would have period one, resulting in the narrow bars of the respective width aligned with the sensory axis.

The resulting modelling errors for the application of the model with different time horizons to the data from biasing-to-the-right sessions is depicted in Figures A.5, A.6, A.7 and A.8, for biasing-to-the-left in Figures A.9, A.10, A.11 and A.12. 'Memory' of the own behaviour $p$ (ordinate in figures) as well as the history $q$ of the series of $\mathrm{T} 2$ locations (abscissa in figures) ranges from one to 30 steps back. Four subfigures in a row represent four sessions of one subject. Colour code is kept constant across for all subfigures (red indicates a high, blue low modelling error). In both figures data from three different subjects (condition: bias to the right) is depicted. The left block of sessions was produced using the dominant hand, the right block using the non-dominant one.

The modelling error diagrams provide an overview of the dynamics of the prior development. High modelling errors for a few steps back in behavioural history but for all depicted window sizes of sensory history (i.e. lines parallel

\footnotetext{
${ }^{4}$ They are so low-dimensional that the estimation of the according ARMA coefficients (cf. Section 6.3.2) would become singular.
} 
to abscissa) indicate a behaviour that is mainly focuses on what has been done in previous time steps throughout the session. This kind of time series is rather easy to predict. If some sensory history is considered, the plot shows an approximately rectangular or triangular shape of the high modelling errors. This kind of more 'grounded' behaviour leads to responsiveness to the bias. Linear predictability is still good. More complex behaviour, such as no final settling with a strategy (which means that there is no convergence to an average ratio of choosing one or the other direction of movement, i.e. $P_{\infty}$ keeps on drifting), results in sudden error in- and decreases in for some window sizes, visually noticeable as 'bands' (cf. first session of the first participant in Figure A.12).

In the right-handed participants and for the majority of the left-handed ones, it can be seen that the modelling error mainly depends on the size of the time window $p$ in the own behaviour. This suggests that the participants mainly focus on their behaviour from previous sessions, and less on the target location that is actually presented.

However, there is a small number of sessions where $q$ does play a role (e.g. in participant MT of the left-handed ones). These participants also produce a lower $P_{\infty}^{x}$. This may rise the idea that this limit probability changes dependent on the intersection with abscissa of a line separating low from high modelling error.

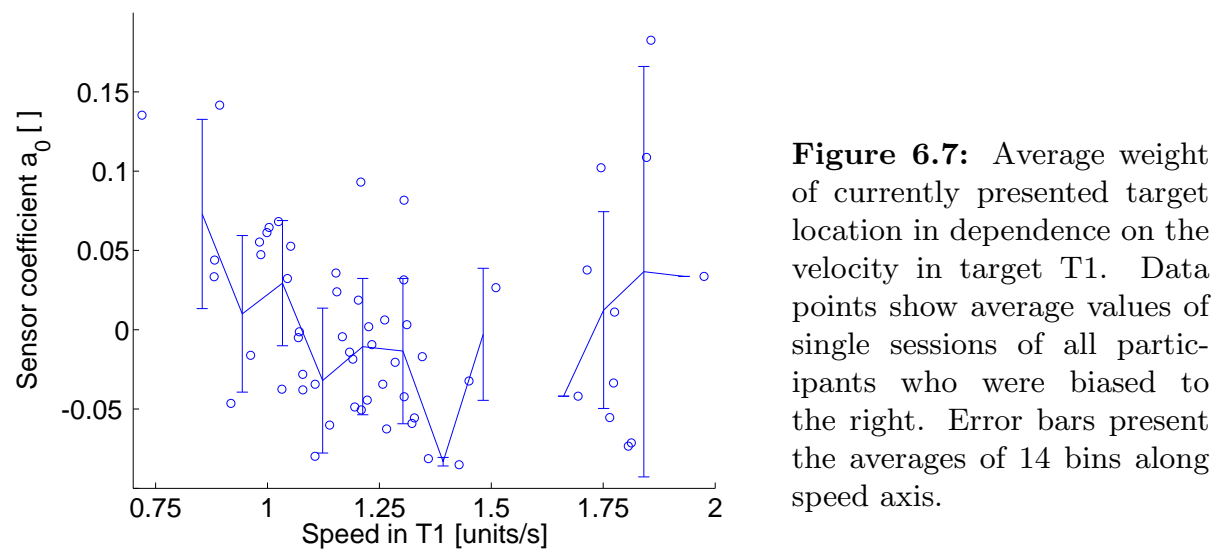

The left subfigure of Figure 6.7 furthermore suggests that there is no direct connection between movement speed in target $\mathrm{T} 1$ and the average responsiveness to the current stimulus. The extent of attention drawn to the perceived location of target T2 in the previous trial drops for speeds higher than 1.2 unit- 
$\mathrm{s} / \mathrm{s}$. The right subfigure supports the criticality of this speed threshold. For slower movements all kind of coefficients (mainly between 0 and 1 since participants tend to follow their previous initial action in at least a fraction of the trials) can occur, showing the individual differences in decision strategy, whereas participants tend to stick stronger to their previous action when executing for faster movements.

Sensor-behaviour tradeoff For a first-order approximation to the behavioural dynamics ARMA was applied with only one step of behaviour and sensor memory $(p=1, q=1)$. Interestingly, in all subjects both resulting sensor coefficients $b_{0}$ and $b_{1}$ are way lower than the behaviour coefficient $a_{1}$. A low $b_{0}$ is to be expected, since it simply indicates that there is not enough time to process the presented location of target T2. In general the large difference $a_{1}-b_{1}$ shows the great emphasis on one's own behaviour. It furthermore relates to the limit probability $P_{\infty}^{x}$ as suggested in Figure 6.8: The greater the focus on one's own behaviour is on average, the larger is $P_{\infty}^{x}$. This gives a possible explanation (or better: hypothesis) for the sensitivity to an initial bias. The participants perceive some properties of the time series and then gets stuck with their "conclusion" from it which then gets amplified in the "behaviour loop" (see Section 6.3.3 for a extension of this idea).

Do modelling errors reveal differences in motor decision strategy? The significant drop of the modelling error for sensor window sizes smaller than 30 (cf. Figures A.5, A.6, A.7 and A.8) in some participants which seems to coincide with a matching $P_{\infty}^{x}$ indicates that they do memorise and consider sequences of perceived stimuli to a certain extend. Intuitively, this fact may result in a less stereotypical choice of movement direction behind target T1.

\subsubsection{Correctness vs. simplicity under time pressure}

Figure 6.8 and 6.9 show the relation between $P_{\infty}$ and the differences $a_{1}-b_{0}$ (left subfigure) and $a_{1}-b_{1}$ (right subfigure). It can be seen that for usage of the right hand when being confronted with a bias to the right, participants easily get stuck with the bias to that side, however, using the left hand does not as frequently lead to susceptibility to the bias. Furthermore, different levels of responsiveness to the bias can be seen. If one thinks of $a_{1}-b_{1}$ as the difference in emphasis between the own behavioural tendency (behind target T1) in the 

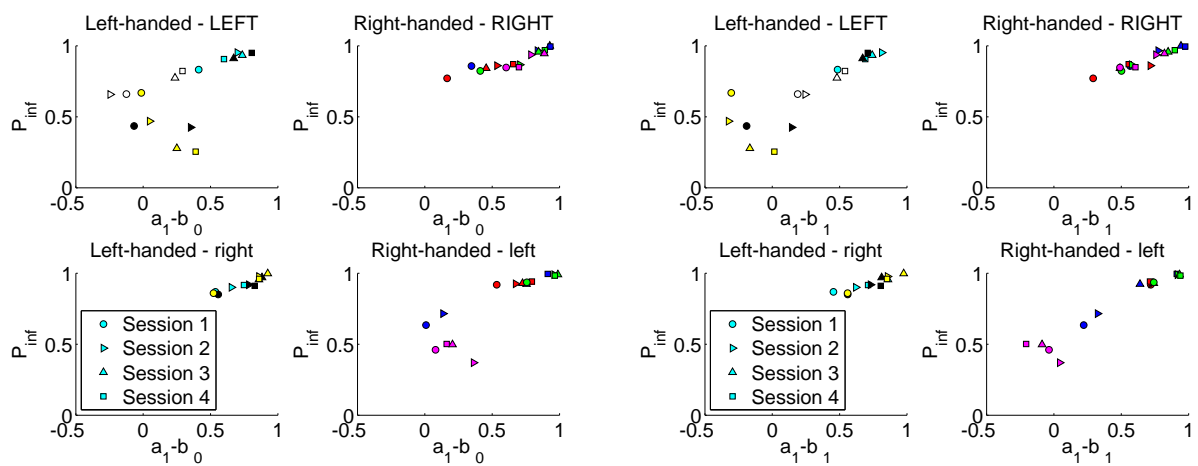

(a) Limit probability over behavioural emphasis relative to current target location

(b) Limit probability over behavioural emphasis relative to previous target location

Figure 6.8: Linear dependence, condition bias to the right: Relation between limit prior and the differences of coefficients from linear prediction of behaviour with $p=1$ and $q=1$. Subsubplots indicate handedness and hand usage: Top left: Left-handed using dominant hand. Top right: Right-handed using dominant hand. Bottom left: Left-handed using non-dominant hand. Bottom right: Right-handed using non-dominant hand.
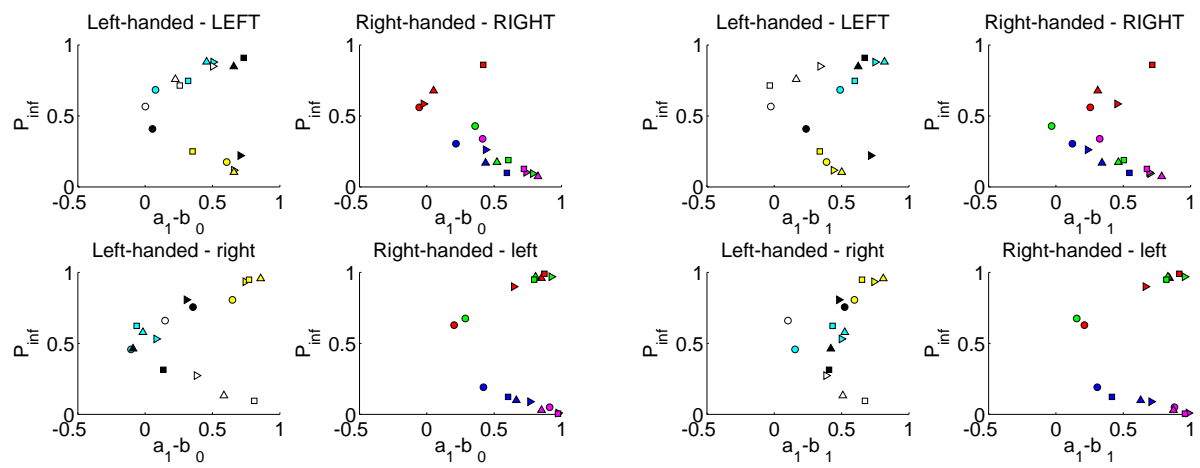

(a) Limit probability over behavioural emphasis relative to current target location

(b) Limit probability over behavioural emphasis relative to previous target location

Figure 6.9: Linear dependence, condition bias to the left: Relation between limit prior and the differences of coefficients from linear prediction of behaviour with $p=1$ and $q=1$. Subsubplots indicate handedness and hand usage: Top left: Left-handed using dominant hand. Top right: Right-handed using dominant hand. Bottom left: Left-handed using non-dominant hand. Bottom right: Right-handed using non-dominant hand. 
previous time step (represented by $a_{1}$ ) and the perception in the previous trial (represented by $b_{1}$ ), there is a connection between this focus on the modality and the frequency with which participants end up to have moved to the right in a session. The stronger they rely on what they have done in the past, the stronger the initial bias is able to influence their behaviour.
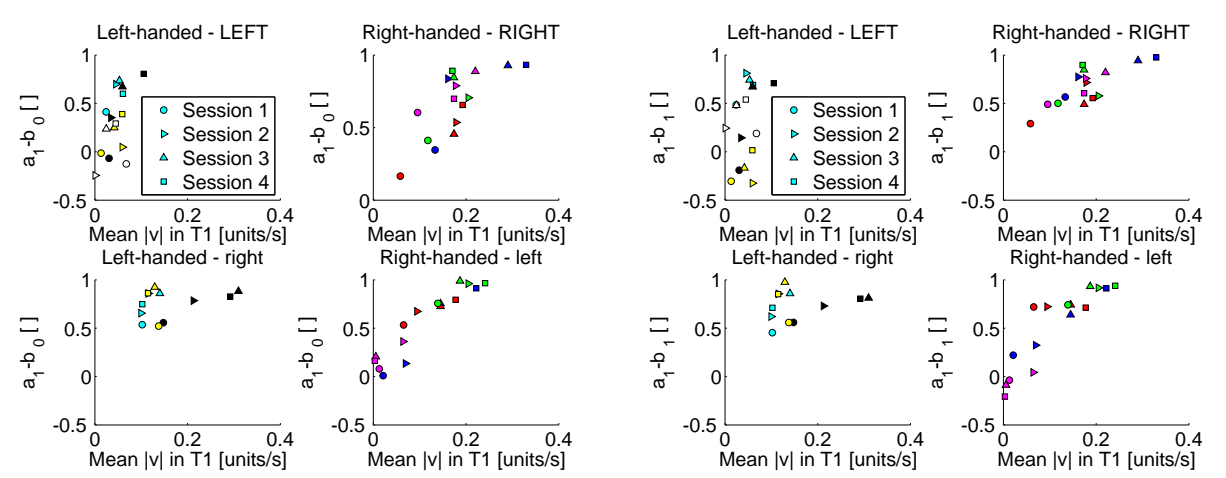

(a) Behavioural emphasis relative to current target location over absolute velocity in $\mathrm{T} 1$.

(b) Behavioural emphasis relative to previous target location over absolute velocity in T1.

Figure 6.10: Condition bias to the right: Relation between the differences of coefficients from linear prediction of behaviour with $p=1$ and $q=0$, and the velocity in target T1. Subsubplots indicate handedness and hand usage: Top left: Left-handed using dominant hand. Top right: Right-handed using dominant hand. Bottom left: Left-handed using non-dominant hand. Bottom right: Right-handed using non-dominant hand. Colour code is used for participants: Right-handed: blue - CK red - EE, green - DB, magenta - KF. Left-handed: cyan - BK, black - SA, yellow MT, white - JS.

Furthermore there is a connection between movement speed and overall limit probability (as Figure 6.12 suggests). From the left subfigure it can be seen that for higher movement speed (above 1.2 units/s) an initial biasing to the left in general leads to an increased preference to move to the left, and initial overemphasising of the $\mathrm{T} 2$ location at the right causes participants to produce more rightwards movements. This can be seen as another hint towards the idea that the strategy (more attention to visual evidence versus greater focus on own behavioural tendencies during previous trials) does influence the final overall behavioural performance.

Furthermore, the right subfigure of Figure 6.12 allows a more detailed analysis of this phenomenon with an eye on handedness and used hand. If the direction of the bias agrees with the used hand in general a behaviour with accordingly absolute values of $P_{\infty}$ is shown. If the direction of bias does not 

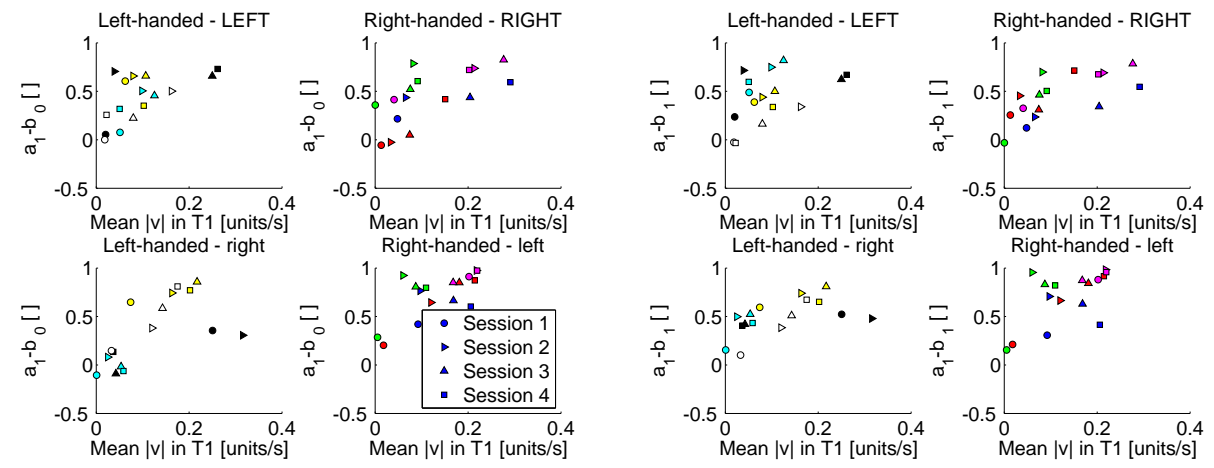

(a) Behavioural emphasis relative to current target location over absolute velocity in $\mathrm{T} 1$.

(b) Behavioural emphasis relative to previous target location over absolute velocity in $\mathrm{T} 1$.

Figure 6.11: Condition bias to the left: Relation between the differences of coefficients from linear prediction of behaviour with $p=1$ and $q=1$, and the velocity in target T1. Subsubplots indicate handedness and hand usage: Top left: Left-handed using dominant hand. Top right: Right-handed using dominant hand. Bottom left: Left-handed using non-dominant hand. Bottom right: Right-handed using non-dominant hand. Colour code is used for participants: Right-handed: blue - DL, red - JA, green - KW, magenta - MB. Left-handed: cyan - BK2, black - MB, yellow RW, white - YB.

coincide with the used hand, only higher velocities result in high (right-biasing) or low (left-biasing) $P_{\infty}$. In this condition slower velocities result in a mixed behaviour with movement preferences in varying degrees of $P_{\infty}$. This suggests that another tradeoff is considered. For example, slower velocities allow sensory information to be considered earlier. ${ }^{5}$

\subsection{4 "Internal" dynamics subserving directional choice}

\section{Nonlinear autoregressive modelling}

The results of an unrestricted parameter optimisation ${ }^{6}$ for $\epsilon, \Lambda$, and $P_{\text {start }}$ as well as the resulting prediction error are summarised in Figure 6.13 for bias to the right, and Figure 6.14 for bias to the left. Subplots contain data in dependence on handedness and used hand: upper row shows parameters for dominant hand, side of columns corresponds to which hand is dominant. Table 6.2 and 6.3 show the exact values, and give an overview of the development of the average

\footnotetext{
${ }^{5}$ In general this is feasible to at least some extend due to the $350 \mathrm{~ms}$ 'long' time window for leaving the proximity of target $\mathrm{T} 1$.

${ }^{6}$ 'Unrestricted' means that $P_{\text {start }}<P_{\infty}$ are admissible.
} 

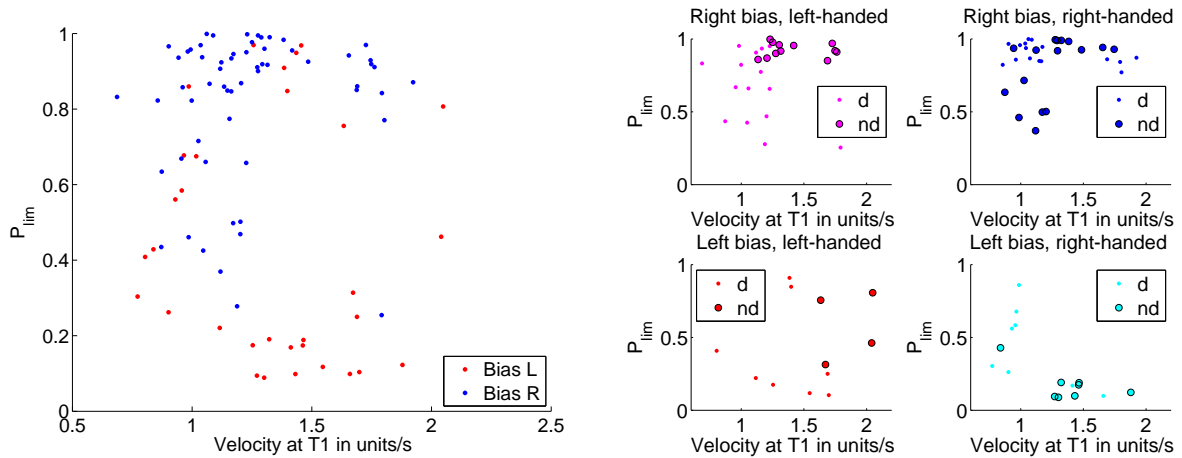

Figure 6.12: Left: Relation between $P_{\infty}^{x}$ and average velocity on arrival in target $\mathrm{T} 1$ for each session by all participants. Data is split by imposed bias: initial bias to the left (red) or to the right (blue). Right: Same as on the left, but split by handedness and used hand: Upper row: Bias to the right. Lower row: Bias to the left. Left column: Left-handed participants. Right column: Right-handed participants. Data from usage of dominant hand is depicted with dots, data from usage of nondominant hand is depicted with circles.

parameters $\epsilon, P_{\infty}$, and $P_{\text {start }}$ over sessions, grouped by handedness and used hand. Figures 6.15 and 6.16 show data and model of the process for bias to the right, Figures 6.17 and 6.18 for bias to the left.

This phenomenological model suggests that there is a stronger influence of the parameter used hand on the behaviour than of the parameter handedness. In the biasing-to-the-right condition participants who use the right hand quicker stick to the bias than the ones using the left hand. This suggests that effects of hand usage dominate the ones of handedness. 

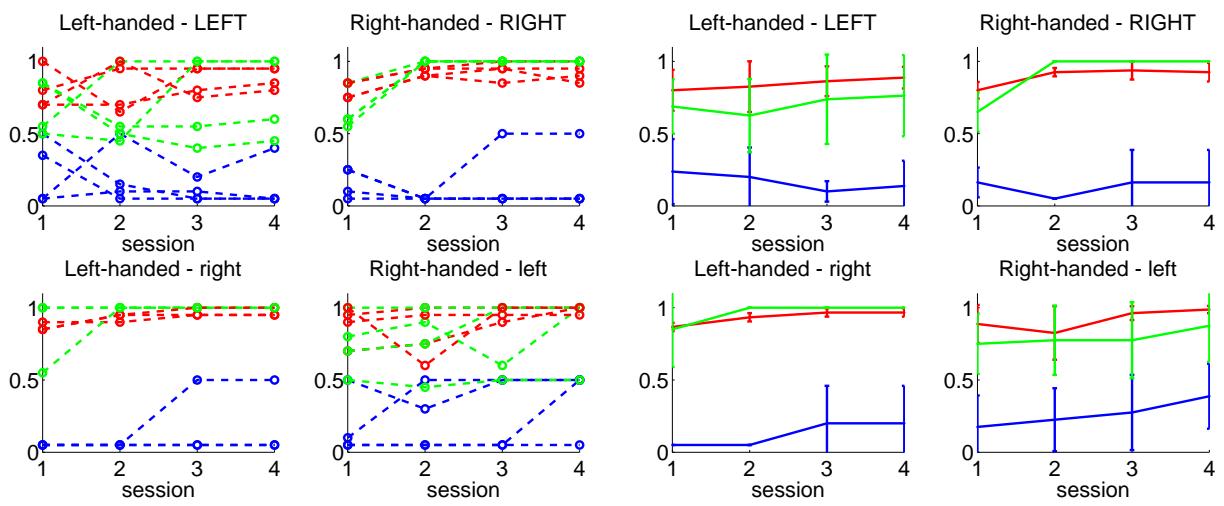

Figure 6.13: initR Left: Model parameters $\epsilon$ (blue), $\Lambda$ (red) and $P_{\text {start }}$ (green) for unrestricted nonlinear dynamics model as a function of sessions. Right: Averages. Left and right subplots are split by handedness and used hand: Upper left subsubplot: lefthanded subjects using their left hand. Upper right subsubplot: right-handed subjects using their right hand. Lower left subsubplot: left-handed subjects using their right hand. Lower right subsubplot: right-handed subjects using their left hand.
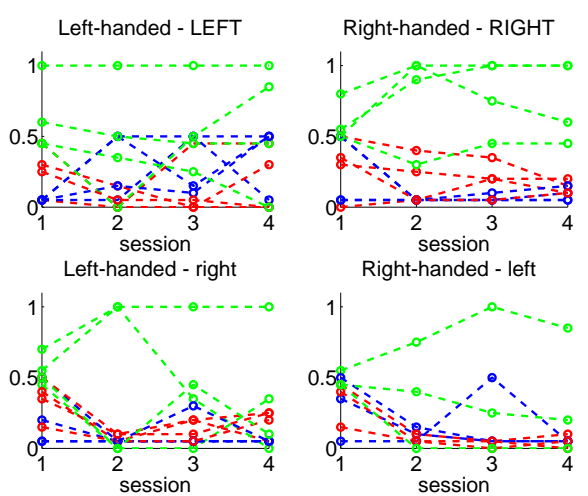

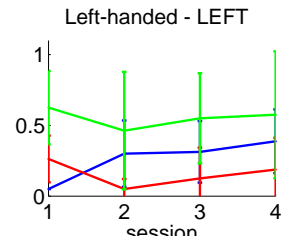

Left-handed - right

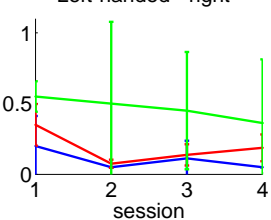

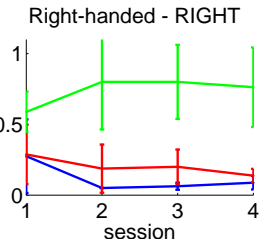

Right-handed - left

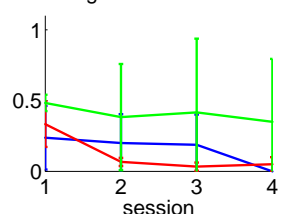

Figure 6.14: initL Left: Model parameters $\epsilon$ (blue), $\Lambda$ (red) and $P_{\text {start }}$ (green) for unrestricted nonlinear dynamics model as a function of sessions. Right: Averages. Left and right subplots are split by handedness and used hand: Upper left subsubplot: lefthanded subjects using their left hand. Upper right subsubplot: right-handed subjects using their right hand. Lower left subsubplot: left-handed subjects using their right hand. Lower right subsubplot: right-handed subjects using their left hand. 
Table 6.2: Accumulative averaging for $P_{r}^{x}$ : Overview of the development of the average parameters $\epsilon, P_{\text {start }}$ and $P_{\infty}$, and summed modelling error over sessions, grouped by handedness and used hand, condition bias to the right. Column " $\mathrm{H}$ " indicates handedness, columns "HU" informs about hand usage.

\begin{tabular}{|l|l|c|c|c|c|}
\hline \multicolumn{5}{|c|}{$\epsilon$} \\
\hline $\mathrm{H}$ & $\mathrm{HU}$ & Session 1 & Session 2 & Session 3 & Session 4 \\
\hline $\mathrm{R}$ & $\mathrm{R}$ & $0.26+/-0.18$ & $0.38+/-0.14$ & $0.47+/-0.17$ & $0.50+/-0.00$ \\
$\mathrm{R}$ & $\mathrm{l}$ & $0.29+/-0.28$ & $0.34+/-0.21$ & $0.39+/-0.43$ & $0.33+/-0.22$ \\
$\mathrm{~L}$ & $\mathrm{~L}$ & $0.42+/-0.29$ & $0.14+/-0.09$ & $0.15+/-0.09$ & $0.19+/-0.17$ \\
$\mathrm{~L}$ & $\mathrm{r}$ & $0.35+/-0.26$ & $0.35+/-0.26$ & $0.33+/-0.14$ & $0.27+/-0.23$ \\
\hline \hline \multicolumn{5}{|c|}{$P_{\text {start }}$} \\
\hline $\mathrm{H}$ & $\mathrm{HU}$ & Session 1 & Session 2 & Session 3 & Session 4 \\
\hline $\mathrm{R}$ & $\mathrm{R}$ & $0.61+/-0.06$ & $0.90+/-0.06$ & $0.91+/-0.07$ & $0.89+/-0.07$ \\
$\mathrm{R}$ & $\mathrm{l}$ & $0.68+/-0.20$ & $0.71+/-0.21$ & $0.75+/-0.23$ & $0.84+/-0.23$ \\
$\mathrm{~L}$ & $\mathrm{~L}$ & $0.56+/-0.05$ & $0.61+/-0.23$ & $0.71+/-0.28$ & $0.74+/-0.25$ \\
$\mathrm{~L}$ & $\mathrm{r}$ & $0.73+/-0.16$ & $0.92+/-0.06$ & $0.95+/-0.00$ & $0.95+/-0.00$ \\
\hline \hline \multicolumn{5}{|c|}{$P_{\infty}$} \\
\hline $\mathrm{H}$ & $\mathrm{HU}$ & Session 1 & Session 2 & Session 3 & Session 4 \\
\hline $\mathrm{R}$ & $\mathrm{R}$ & $0.80+/-0.06$ & $0.93+/-0.03$ & $0.94+/-0.06$ & $0.94+/-0.08$ \\
$\mathrm{R}$ & $\mathrm{l}$ & $0.77+/-0.18$ & $0.83+/-0.18$ & $0.85+/-0.20$ & $0.88+/-0.22$ \\
$\mathrm{~L}$ & $\mathrm{~L}$ & $0.69+/-0.10$ & $0.71+/-0.17$ & $0.86+/-0.10$ & $0.89+/-0.07$ \\
$\mathrm{~L}$ & $\mathrm{r}$ & $0.87+/-0.03$ & $0.95+/-0.05$ & $0.97+/-0.03$ & $0.97+/-0.03$ \\
\hline \hline \multicolumn{7}{|c|}{ error } \\
\hline $\mathrm{H}$ & $\mathrm{HU}$ & Session 1 & Session 2 & Session 3 & Session 4 \\
\hline $\mathrm{R}$ & $\mathrm{R}$ & $11.17+/-7.83$ & $7.38+/-1.28$ & $4.95+/-3.81$ & $5.97+/-3.63$ \\
$\mathrm{R}$ & $\mathrm{l}$ & $12.63+/-8.32$ & $6.21+/-3.68$ & $7.32+/-4.07$ & $4.95+/-3.75$ \\
$\mathrm{~L}$ & $\mathrm{~L}$ & $13.11+/-5.16$ & $13.83+/-9.64$ & $6.90+/-0.86$ & $8.60+/-2.63$ \\
$\mathrm{~L}$ & $\mathrm{r}$ & $7.79+/-1.41$ & $7.15+/-0.90$ & $3.60+/-3.03$ & $8.19+/-1.78$ \\
\hline
\end{tabular}


Table 6.3: Accumulative averaging for $P_{r}^{x}$ : Overview of the development of the average parameters $\epsilon, P_{\text {start }}$ and $P_{\infty}$, and summed modelling error over sessions, grouped by handedness and used hand, condition bias to the left. Column " $\mathrm{H}$ " again indicates handedness, columns "HU" informs about hand usage.

\begin{tabular}{|l|l|c|c|c|c|}
\hline \multicolumn{5}{|c|}{$\epsilon$} \\
\hline $\mathrm{H}$ & $\mathrm{HU}$ & Session 1 & Session 2 & Session 3 & Session 4 \\
\hline $\mathrm{R}$ & $\mathrm{R}$ & $0.35+/-0.37$ & $0.52+/-0.09$ & $0.29+/-0.25$ & $0.30+/-0.23$ \\
$\mathrm{R}$ & $\mathrm{l}$ & $0.28+/-0.19$ & $0.34+/-0.20$ & $0.50+/-0.00$ & $0.50+/-0.00$ \\
$\mathrm{~L}$ & $\mathrm{~L}$ & $0.35+/-0.27$ & $0.50+/-0.00$ & $0.34+/-0.21$ & $0.31+/-0.21$ \\
$\mathrm{~L}$ & $\mathrm{r}$ & $0.35+/-0.35$ & $0.19+/-0.21$ & $0.08+/-0.03$ & $0.26+/-0.25$ \\
\hline \hline \multicolumn{5}{|c|}{$P_{\text {start }}$} \\
\hline $\mathrm{H}$ & $\mathrm{HU}$ & Session 1 & Session 2 & Session 3 & Session 4 \\
\hline $\mathrm{R}$ & $\mathrm{R}$ & $0.36+/-0.21$ & $0.29+/-0.23$ & $0.33+/-0.15$ & $0.34+/-0.18$ \\
$\mathrm{R}$ & $\mathrm{l}$ & $0.37+/-0.24$ & $0.42+/-0.47$ & $0.37+/-0.51$ & $0.38+/-0.49$ \\
$\mathrm{~L}$ & $\mathrm{~L}$ & $0.60+/-0.24$ & $0.47+/-0.43$ & $0.54+/-0.32$ & $0.61+/-0.29$ \\
$\mathrm{~L}$ & $\mathrm{r}$ & $0.54+/-0.06$ & $0.60+/-0.16$ & $0.53+/-0.17$ & $0.57+/-0.26$ \\
\hline \hline \multicolumn{5}{|c|}{$P_{\infty}$} \\
\hline $\mathrm{H}$ & $\mathrm{HU}$ & Session 1 & Session 2 & Session 3 & Session 4 \\
\hline $\mathrm{R}$ & $\mathrm{R}$ & $0.72+/-0.19$ & $0.81+/-0.17$ & $0.80+/-0.12$ & $0.86+/-0.05$ \\
$\mathrm{R}$ & $\mathrm{l}$ & $0.80+/-0.18$ & $0.97+/-0.03$ & $0.98+/-0.03$ & $0.95+/-0.05$ \\
$\mathrm{~L}$ & $\mathrm{~L}$ & $0.78+/-0.18$ & $0.92+/-0.03$ & $0.86+/-0.07$ & $0.81+/-0.09$ \\
$\mathrm{~L}$ & $\mathrm{r}$ & $0.69+/-0.06$ & $0.76+/-0.17$ & $0.74+/-0.22$ & $0.80+/-0.18$ \\
\hline \hline \multicolumn{5}{|c|}{ error } \\
\hline H & HU & Session 1 & Session 2 & Session 3 & Session 4 \\
\hline $\mathrm{R}$ & $\mathrm{R}$ & $15.71+/-2.76$ & $9.69+/-3.43$ & $7.90+/-2.90$ & $9.52+/-3.39$ \\
$\mathrm{R}$ & $\mathrm{l}$ & $17.77+/-16.01$ & $4.39+/-2.92$ & $5.07+/-2.24$ & $4.99+/-3.93$ \\
$\mathrm{~L}$ & $\mathrm{~L}$ & $9.49+/-2.21$ & $12.63+/-6.60$ & $11.02+/-2.95$ & $11.01+/-5.61$ \\
$\mathrm{~L}$ & $\mathrm{r}$ & $19.88+/-6.21$ & $12.75+/-5.89$ & $11.00+/-5.03$ & $10.11+/-7.76$ \\
\hline
\end{tabular}



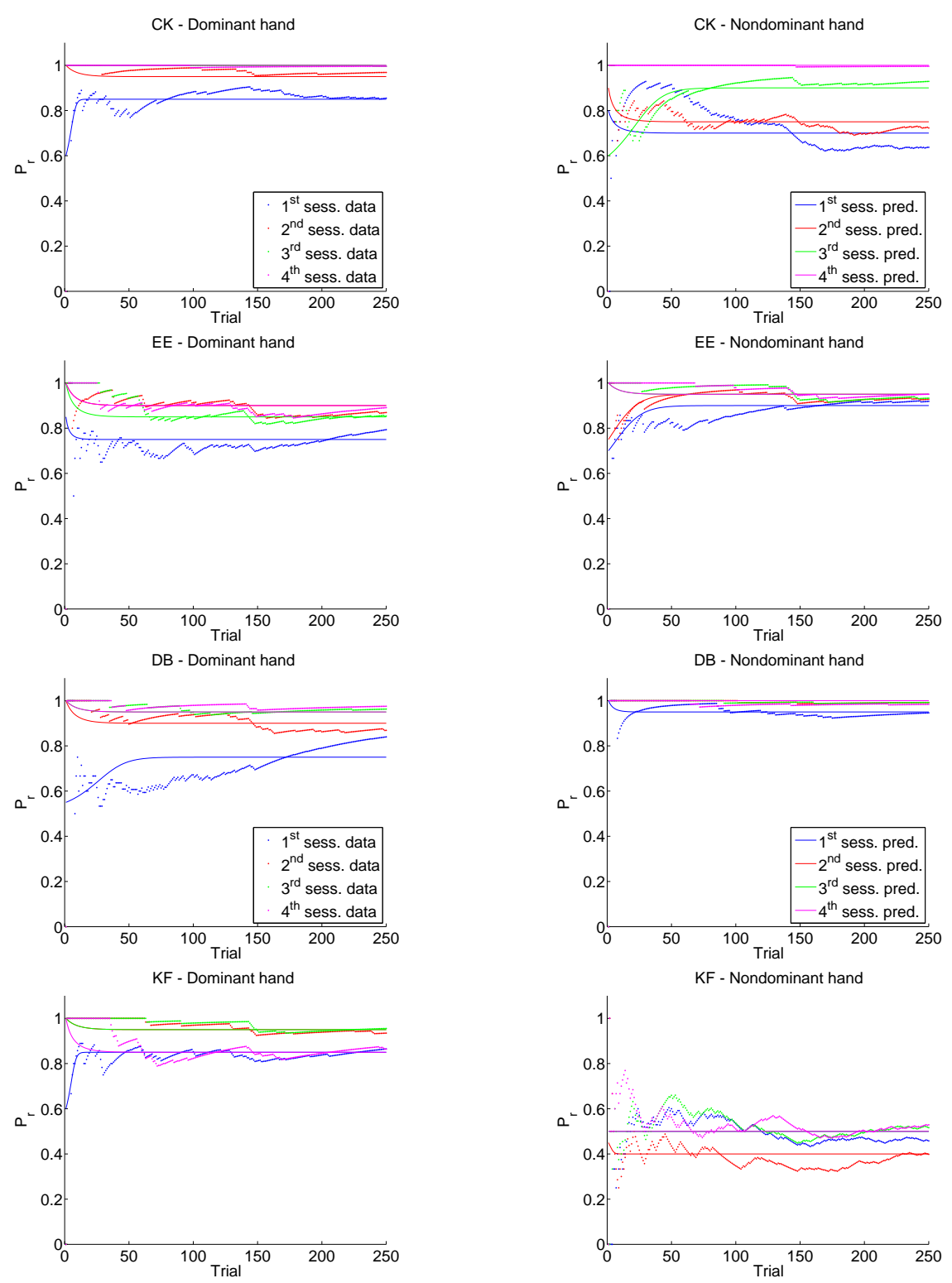

Figure 6.15: Nonlinear modelling of prior in right-handed subjects (limited to 250 trials), condition: bias to the right. Dots - real prior. Line - model. Four sessions per subfigure $\left(1^{\text {st }}-\right.$ blue, $2^{\text {nd }}-$ red, $3^{\text {rd }}-$ green, $4^{\text {th }}-$ magenta $)$. Left column contains data from dominant hand of participant, right column data from non-dominant hand. Each row is a new participant (CK, EE, DB, KF). 

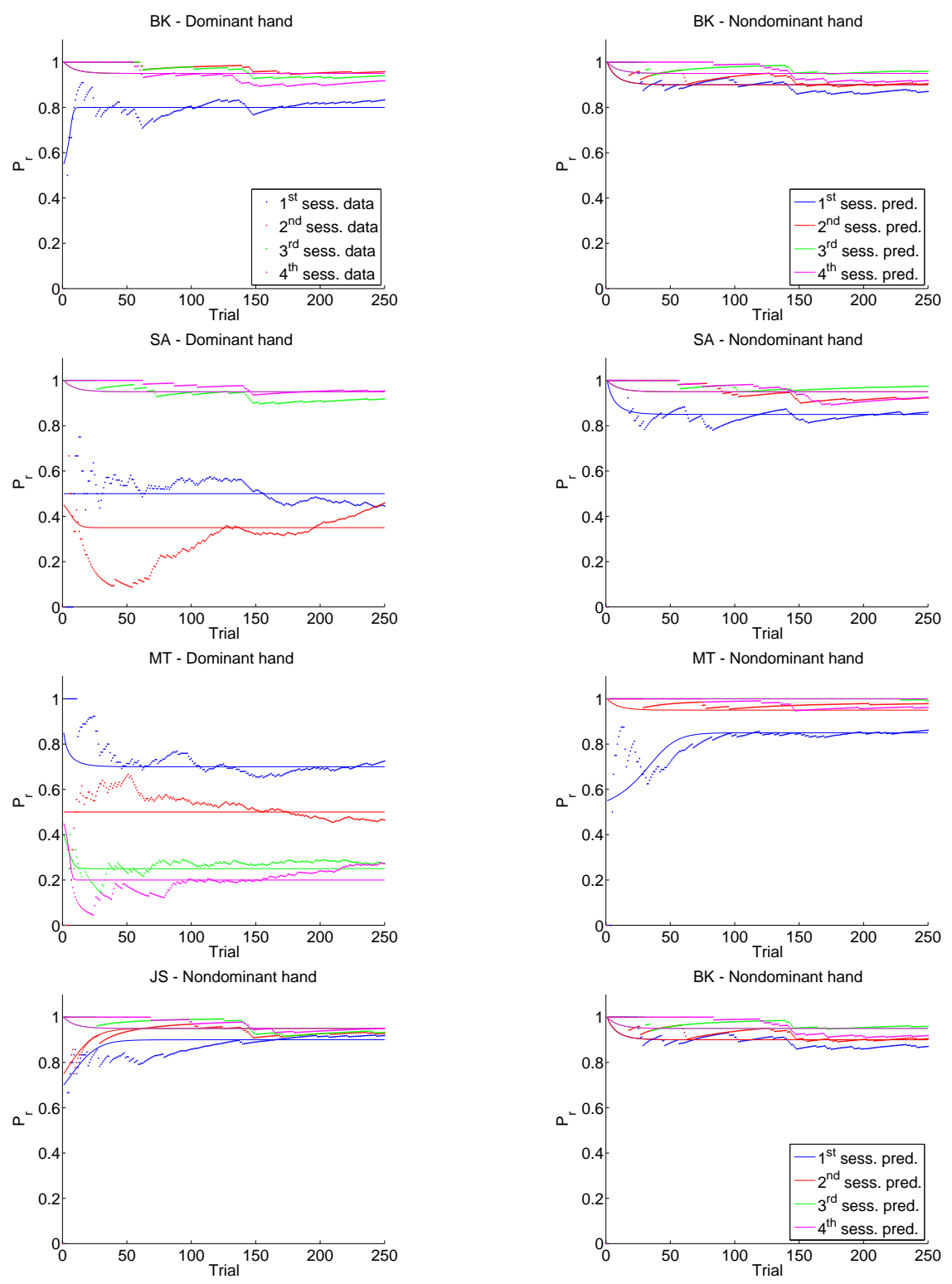

Figure 6.16: Nonlinear modelling of prior in left-handed subjects (limited to 250 trials), condition: bias to the right. Dots - real prior. Line - model. Four sessions per subfigure $\left(1^{\text {st }}-\right.$ blue, $2^{\text {nd }}-$ red, $3^{\text {rd }}-$ green, $4^{\text {th }}-$ magenta $)$. Left column contains data from dominant hand of participant, right column data from non-dominant hand. Each row is a new participant (BK, SA, MT, JS). 
$\mathrm{DL}$ - Dominant hand
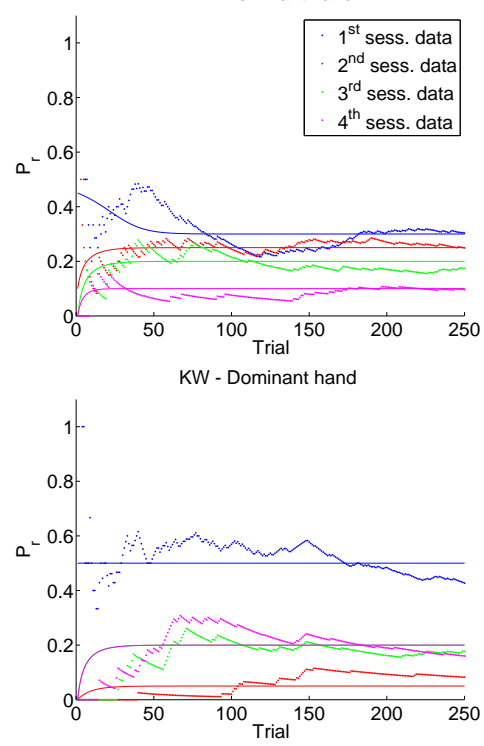

MB - Dominant hand
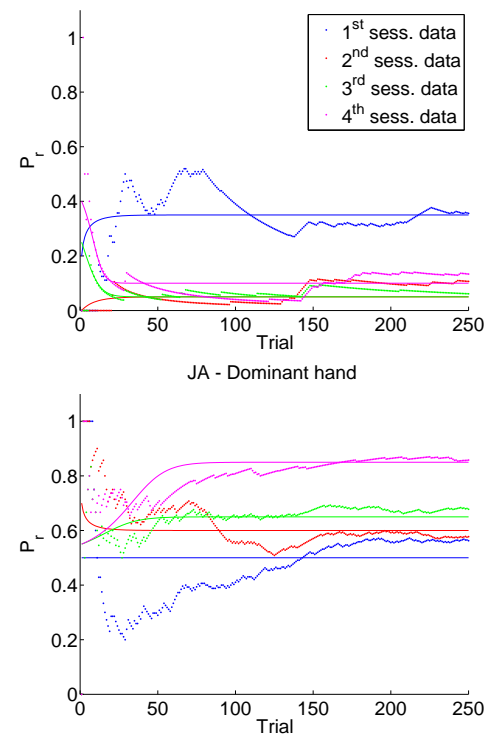

$\mathrm{DL}$ - Dominant hand

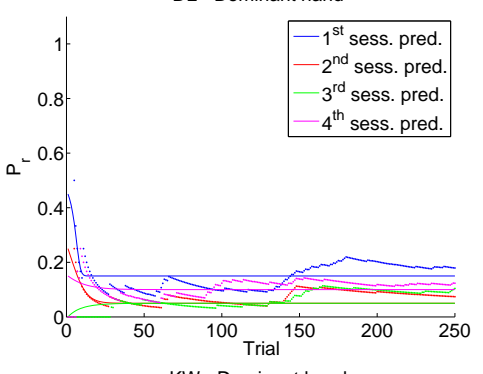

KW - Dominant hand

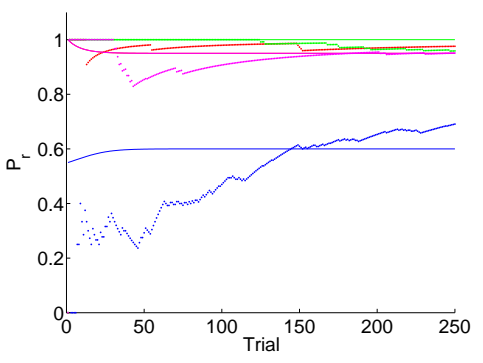

MB - Dominant hand

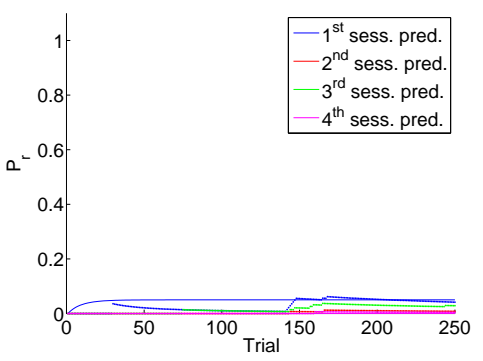

JA - Dominant hand

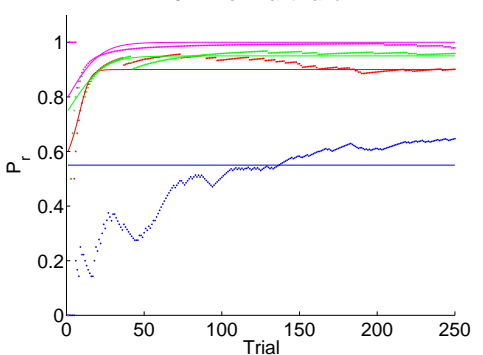

Figure 6.17: Nonlinear modelling of prior in right-handed subjects (limited to 250 trials), condition: bias to the left. Dots - real prior. Line - model. Four sessions per subfigure $\left(1^{\text {st }}-\right.$ blue, $2^{\text {nd }}-$ red, $3^{\text {rd }}$ - green, $4^{\text {th }}$ - magenta $)$. Left column contains data from dominant hand of participant, right column data from non-dominant hand. Each row is a new participant (DL, KW, MB, JA). 

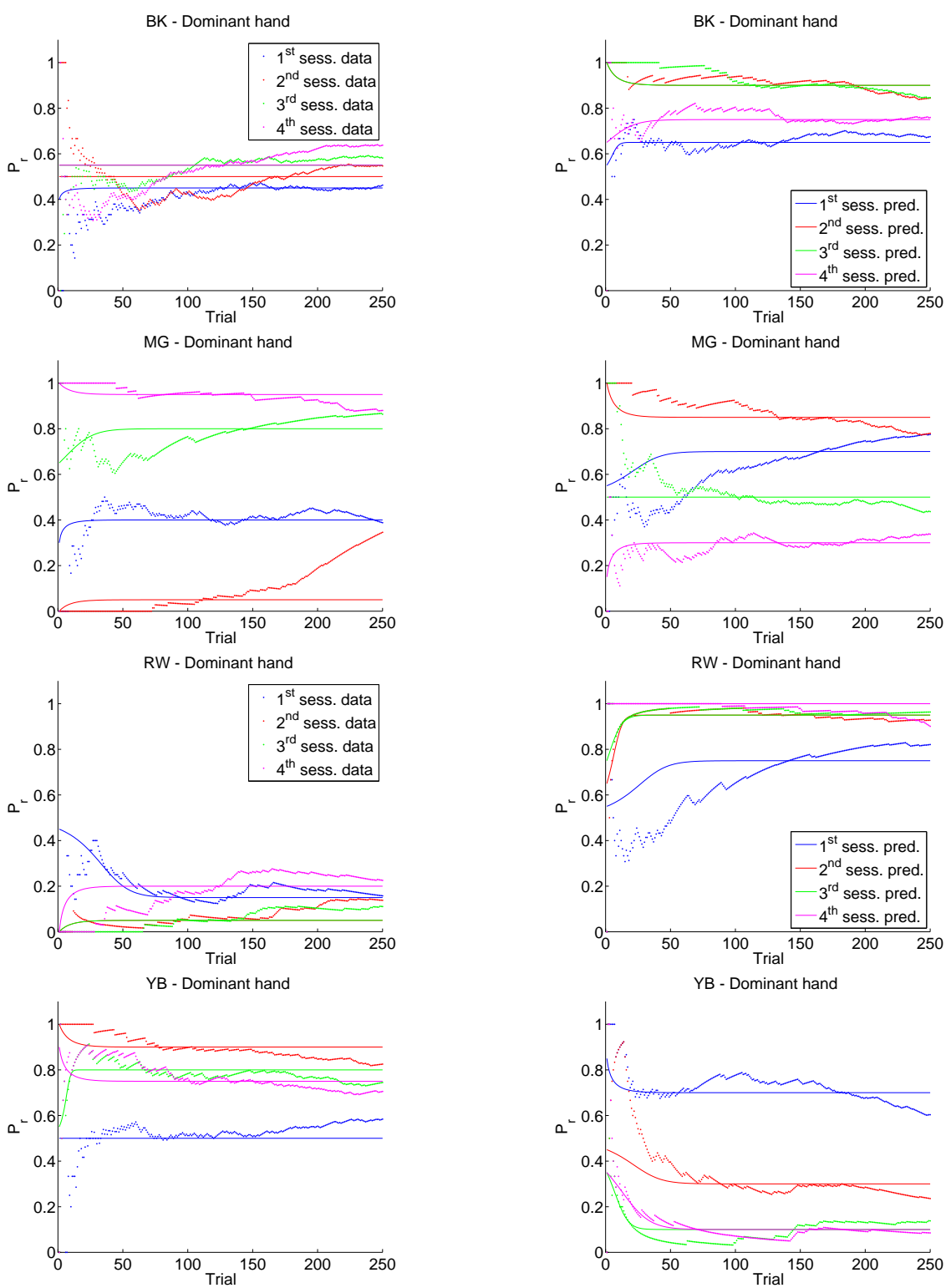

Figure 6.18: Nonlinear modelling of prior in left-handed subjects (limited to 250 trials), condition: bias to the left. Dots - real prior. Line - model. Four sessions per subfigure $\left(1^{\text {st }}-\right.$ blue, $2^{\text {nd }}-$ red, $3^{\text {rd }}-$ green, $4^{\text {th }}-$ magenta $)$. Left column contains data from dominant hand of participant, right column data from non-dominant hand. Each row is a new participant (BK2, MG, RW, YB). 


\subsection{Summary of event-based analysis}

Since the participants' motor decisions had to be made in a limitted amount of time, not only "classical" dimensions like reliability of sensory data has to be considered but also the individual internal "idea" about the scene plays an important role. Participants did not have enough time for sensing carefully, so they had to guess based on the information accumulated during previous trials.

Interestingly, it turned out that there is no direct reference to these previously presented target locations (and also not to sliding averages of the occurance). Instead, stereotypical choice pattern which are grounded in the own behavioral history emerged and manfested throughout the sessions.

The faster the movements were executed - which coincides with less time available for proper processing of the real locations of the final movement target - the stronger particpants preferred a single movement direction (to the left, or to the right). For an initial overemphasis the right target position all participants consistently got stuck with this direction, if the showed stereotypicity. For an initial overrepresentation of final movement targets on the left side behind the obstacle only a fraction of all "biased" participants chose to stick to this side. This phenomenon appeared to be independent of handedness and used hand. This may give rise to the conjecture, that the "defualt" direction is chosen according to our experience in everyday life where in our Western culture $^{7}$ the direction towards the right is emphasised strongly (reading direction, pointing of axes and timelines).

Linear modelling revealed that those who do not end up completely biased, show a pattern in their prior evolution that is not linearly capturable with a consistent performance (cf. Section 6.4.2). Due to the complexity in their strategy and possibly ongoing strategy changes, also the non-linear model shows medium performance.

Under time pressure participants showed a partial neglect of sensory - here: visual - information and a focus on the own rather repetetive behaviour. This suggests that motor decisions are not only based on sensory data and according reliability estimates in combination with costs of potential movements. The decisions are but also influenced by task restrictions and "heuristics" which may be difficult to be implemented in terms of Bayesian decision theory.

\footnotetext{
${ }^{7}$ Most of the participants came from Germany, one from Belarus, and one from Turkey.
} 
The results suggest that in cases of time pressure only a tiny patch of information about the environment is picked up and then amplified to serve as a decision heuristics, if the according costs for replanning are bearable.

Major findings of this study are:

1. Repetitious tasks are solved by withdrawing perceptual attention and substituting it by simpler internal dynamics as a first approximation to the external demands and according error corrections if necessary. (Sections 6.4.2 and 6.4.3)

2. Decisions can be influenced by small biases in the presented stimuli. (Section 6.4.1)

3. The processes underlying this evolution of a prior that allows this kind of biasing can be accomplished using a nonlinear model of the symmetry breaking in the decision task. 


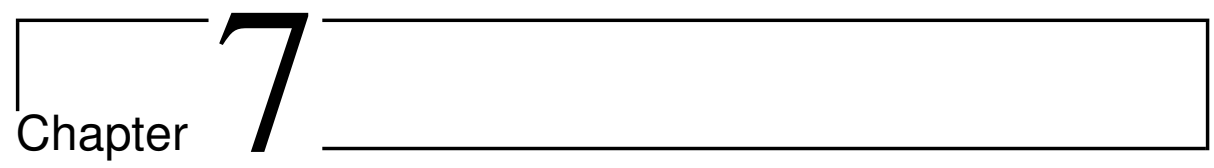

\section{Overall summary}

Humans constantly interact with their environment for instance by producing a vast variety of arm postures and movements. Besides spatial and temporal accuracy often the stability against external disturbances plays an important role in the complex tasks of everyday life. Arm stiffness is an important quantity to study endeffector stability. It quantifies the relation between the force strength of the external disturbance and the resulting displacements of the limb, hence, it summarises the aspects of the spring-like behaviour of the limb. Classically, it was estimated in single joint postures (Feldman, 1966; Bizzi et al., 1984) and in postures throughout a horizontal plane in front of the shoulder (Mussa-Ivaldi et al., 1985). Further approaches examined the stability during planar movements and, apart from stiffness, also identified inertia and viscosity (Tsuji et al., 1995; Gomi and Kawato, 1996). By utilizing a rotatable plane as measurement space stiffness estimates in three dimensions became possible (cf. Acosta et al., 2000). However, due to the complex custom-made setup it is difficult to transfer this method to other fields of application in other places.

For studies of arm postures throughout the natural workspace it is desirable to assess stiffness patterns in more than two dimensions. The resulting knowledge about arm stiffness in 3D not only may help to understand the utilisation of the multiple degrees of freedom in the human arm during simple tasks of everyday life, but also serve as basis for movement studies where the role of dynamic stability is explored in the interplay between static stiffness components and dynamically arising forces. Unfortunately, only few labs, who may be interested in combining stiffness measurements with other techniques of studying the 
human sensorimotor system, are equipped with such highly developed custommade devices required for existing approaches. The development of simplified measurement technique, which could be implemented rather easily in different labs, would help to integrate stiffness into various setups targeting other motor control related aspects (for example by means of TMS).

These two key points - the need for a third dimension in stiffness measurements and the need for a transportable setup using out-of-the-shelf device incited me to come up with a stiffness measurement method, which can be used in the redundant arm to assess endeffector stiffness in a Cartesian coordinate frame attached to the hand. I developed a protocol that uses force-induced displacements into twelve directions in hand space, and a method of assessing the reliability of the estimated stiffness matrix.

It is not trivial that such a procedure works in the redundant human arm for the following two reasons: Firstly, in the majority of the experiments only the hand position was defined. There were no further instructions how to choose the missing degree of freedom ${ }^{1}$. Secondly, the probands were not even pointed to this underdetermination. Interestingly, it turned out that all subjects perfectly managed to consistently repeat the same posture given "natural defaults" over and over again throughout 120 repetitions for a given hand location (see Sections 2.3.2 and 3.2.1).

Moreover, further restrictions to achieve a completely defined arm posture lead to severe difficulties in the task. Under these circumstances, subjects found it difficult to repeat the measurement even only 60 times per given posture. The additional refinement in posture definition seems to intervene in natural fatigue compensation mechanisms. This finally leads to a reduced operationality of the according "posture protocol", so that can be absolved only by athletes (cf. Section 3.2.4. Nevertheless, the "position protocol" works fine also in naïve subjects under different degrees of distraction from the actual motor task.

Two methods of distraction were tested: a) counting "one, two, three" and b) reading English words from the screen. Both were successfully used in more than 10 participants. The major distinction between counting and reading was the completion time of the task. While counting on average could be finished within 2 to $2.5 \mathrm{~s}$, for reading a time of up to $3 \mathrm{~s}$ had to be granted. These differences in temporal performance can serve as a guideline for future applications of the presented task variations: In challenging experiments producing massive

\footnotetext{
${ }^{1}$ Joint space in the experiments comprised three degrees of freedom at the shoulder and one at the elbow.
} 
amounts of data per participant, it is more appropriate to limit the distraction task to counting to give the subjects time to keep their state of motivation constant. On the other hand, in experiments that aim to show regularities across a big population of individuals, the reading task should be employed. This would foster the interestingness of the experiment and also disguise the actual variables of interest.

It should be remembered that arm stiffness is rather fragile to voluntary corrections by the participants in case of a strong focus on what is going on with their hand. Unfortunately, this vulnerability cannot be excluded from the presented method. Any improvement in this context would require the production of relatively high forces at high frequencies, as done by Acosta et al. (2000). But even these approaches still suffer from the susceptibility to cocontraction and other voluntarily controllable and even subconsciously produced ${ }^{2}$ changes in muscle activation, which then directly influence arm stiffness.

The application of the introduced method using predefined hand positions in the natural workspace of the human arm lead to stiffness estimates that show a strong position dependence. This is consistent with classical studies (Mussa-Ivaldi et al., 1985) that stated the dependence of arm stiffness on arm configuration. But in contrast to those studies on the human arm kept in a horizontal plane at shoulder height I found endeffector stiffness aligned with forearm direction rather than with the hand-shoulder axis. This suggests a low effect of elbow stiffness and two-joint muscle contribution to the overall stability, while a higher stiffness is kept at the shoulder. This finding is consistent with a recent study by Krutky et al. (2010), who mainly concentrate on the sensitivity of stretch reflexes in the human arm to interactions between limb and environmental mechanics. The difference between stiffness orientation in an arm restricted to a plane and an arm acting in the natural workspace is likely to be modulated by the different activation pattern of the involved muscles. In planar studies the arm was supported by a rope from the ceiling, which carried a significant amount of the weight of the arm so that muscles mainly reacted to externally imposed changes in their length (Flash and Mussa-Ivaldi, 1990). Unfortunately, I could not experimentally tackle this question of the involvement of different groups of muscles in supported versus unsupported postures in the horizontal plane at shoulder height, because the unsupported condition would require a number of highly trained participants, who can stand the highly

\footnotetext{
${ }^{2}$ Such alterations, for example, can be made in reaction to individually perceived instabilities, as the studies in Franklin et al. (2007) suggest.
} 
repetitive procedure in arm postures which usually are not adopted in everyday life.

In another set of experiments I examined the effect of a constantly acting external force field on arm stiffness. Flash and Gurevich (1991) suggested that the additionally arising torques are compensated at joint level by a linear superposition of the individual joint contributions. They showed that this mechanism results in an unchanged endeffector stiffness compared to force-free cases. Crucially, their modelling was conducted in joint space. The calculation of the joint angles corresponding to a given hand displacement is straightforward in the nonredundant case. In the redundant case additional assumptions about the choice of the "missing" degree of freedom would have to be made to derive joint angles as well as a regularised inverse of the Jacobian for stiffness transformation (cf. Section 2.2.3). In my experiments endeffector stiffness indeed turned out to be invariant under external force fields of different strengths and directions (cf. Section3.2.3).

Although participants did not perceive the procedure as severely more tiring, data showed an increased variability resulting in less reliable stiffness estimates. For a transformation of the results into joint space reliable estimates of the joint angles are needed. In principle this is accomplishable, since the manipulandum tracks position and orientation of the handle. Yet practically the transformation of these data to joint angle estimates does not lead to the desired results. The non-neglectable variability in nevertheless consistent of grasping the handle of the machine ${ }^{3}$ results in a variability in handle orientations, which then is further amplified by the nonlinear transformation into joint space of the human arm. An additional source of uncertainty are the anthropomorphic measurements, which have to be carried out to assess a) the exact location of the participants shoulder relative to the machine, b) upper arm length, and c) forearm length. Taken together, the resulting variability countermines further analysis of the recorded stiffness patterns in joint space.

This limitation to the applicability of the presented approach - when it comes to high precision joint space analysis - can be compensated by additional joint angle estimation using optical tracking methods ${ }^{4}$. Therefore, the developed technique of stiffness measurements could be an interesting augmentation of studies that deal with motor control related questions in laboratories that

\footnotetext{
3 "Consistent" shall be understood according to the definition in Equation 2.1.

${ }^{4}$ Due to the massive amount of metal used in the SensAble Phantom 3.0 6DoF one has to refrain from using magnetic trackers.
} 
are not exclusively focused on high-precision stiffness estimates but more interested in the general picture how stiffness varies in the execution of tasks of the everyday life and how according stiffness patterns then could be utilised for robot control. The knowledge about naturally arising static and dynamic stability and how it can be utilised for facilitating the successful completion of specific actions is of great importance to the safety and acceptability of robotic systems. By providing means to assess endeffector stiffness in three-dimensional space even in laboratories that are not specialised on high-performance stiffness measurements the approach, which I presented, can help to increase this safety and acceptability.

The motor systems of higher life forms is highly organised and able to generate appropriate movements in a vast variety of situations. This flexibility and optimality always stimulated robotic approaches and already resulted in a high number of fascinating applications, like the salamandra robot using neuromechanical simulations by Ijspeert (2001) or the RunBot, which is the world's fastest running robot, using a combination of reflexive and adaptive neuronal control schemes by Manoonpong et al. (2007).

Control strategies employed by the human motor system may especially provide new ideas for control principles of prostheses and even robot arms. To appropriately study various characteristics of human arm movement an adequately configurable framework is needed. For my studies presented in this thesis a toolbox for the generation of various movement tasks in three-dimensional endeffector space was developed (cf. Section 4.2). This toolbox contains a number of preconfigured experiments involving point-to-point and via-point movements as well as a a custom-made script language that can be used to configure own experimental paradigms. (cf. Section A.4.2). The resulting visual scene is rendered as a virtual reality and can be displayed on almost arbitrary devices ${ }^{5}$.

Usually computational models of human decision behaviour take a rather long time until they produce the desired output, because computation time increases exponentially with the complexity of the decision problem. Their performance can be enhanced by the usage of heuristics. These strategies serve as guidelines for good decisions, e.g. by providing optimised starting values for employed algorithms, and are often inspired by human decision behaviour. In

\footnotetext{
${ }^{5}$ For the presented experiments a computer screen was used. In a number of further experiments also head-mounted video glasses showed a great performance and helped to suppress visual feedback of the arm.
} 
robotics, control principles often rely on a full model of the available hardware (or body) and decisions have to be made on the basis of numerical solutions of the according differential equations. If time is limited these approaches may reach their limits in terms of computation time. Heuristics, which could serve as sources of naturally inspired default values, may help at this point. They can be derived from the optimal solutions humans come up with in motor tasks under time pressure.

The study presented in this thesis targets at understanding such "heuristics" in human decision behaviour. For this purpose a two-alternative forced-choice motor task was implemented in the above mentioned framework. Participants performed obstacle avoidance in combination with a via-point task. Each trial started with a downward movement towards an intermediate target. When arriving at the via-point the location of the final target is revealed which indicates whether the obstacle was to be circumvented on its right or left side. The task was spatially symmetric. The participants had to take the shortest way towards the final movement target. Movements that were initiated into the wrong direction had to be corrected by a turnaround. Providing information that is crucial for the successful completion of the trial at a well-defined instant of time helped to disentangle the participants initial idea about the final movement target location and their sensory-motor reaction to the actually presented one, especially in cases of erroneous decisions. To induce time pressure during execution of the trials a "time tolerance band" was introduced. This rectangular area around the intermediate target had to be left within $350 \mathrm{~ms}^{6}$.

In this setup decision behaviour may have been influenced by several parameters, of which I will only name some in the following. The frequency of occurrence of the final movement target certainly plays a role. There also may be hardware-related preferences, such that the usage of the right hand may enforce movements to the right, and vice versa. An additional regulating parameter could be the time available for the decision, which also may also be associated with the reliability of the gathered information. A further source of influence is the complex phenomenon of history-dependence in the system. Current decisions may be affected by the stimuli that have been perceived and movements that have been executed in the past. There are different ways how the system could keep track of this history: Evidence needed for decisions could

\footnotetext{
${ }^{6}$ This time threshold was experimentally derived and matched best the tradeoff between urging the participant to move smoothly through the via-point and causing frustration because of too strict requirements for the successful completion of trials.
} 
be accumulated a) in total, or b) over a finite time window. Furthermore, effects of forgetting - which would downscaling of the importance of "older" information - could be present in various possible "implementations". For combinatory reasons only a small fraction of combinations of the above mentioned aspects could be considered in the present study.

Due to the strong history-dependence of the human motor system - ranging from learning effects to altered modulation of contractile response of muscle fibres in dependence on their previous activation (cf. Nigg et al., 2000)- I decided to concentrate on the processing of the presented target locations and the role of perceptual and behavioural history. For this reason the time series of presented locations of the final movement target was chosen to be "balanced", that means final movement targets occurred equally often on the left and on the right side behind the obstacle. Moreover, the exact structure of the $\operatorname{long}^{7}$ time series was kept constant across sessions and participants. This way I excluded averaging phenomena due to different local subsequences. The exact "time course" of the series was chosen such that it contained an initial overrepresentation of one target location during the first tens of trials (sessions with overhang to the left were called "initL" and with an overhang to the right "initR"). To study the role of handedness and hand usage all experiments were conducted in left- as well as right-handed individuals, both using their dominant and non-dominant hand.

First of all, participants managed to mainly produce successful trials (with on average only five percent invalid ones) but were not able to report about the shape of the tolerance region. They only reported an overall feeling of rush or time pressure. But although all participants were confronted with the same time restriction they came up with a spectrum of "solutions" to the motor task. When taking the average horizontal velocity in the via-point as a measure of execution speed ${ }^{8}$ and, furthermore, considering the peak velocities of the direct movements around the obstacle it can be seen that usually slow passage of the intermediate target coincided with lower peak velocities, and faster passage was accompanied by high higher ones (cf. Section 5.4.1). However, the average peak velocities seemed to have an upper bound of $1.5 \mathrm{units} / \mathrm{s}$ in all participants of both experimental conditions no matter how fast they passed the intermediate target. Similar limits also hold for the average peak velocities of excursions

\footnotetext{
${ }^{7}$ More than 250 trials in each session (cf. Section 4.3.4).

${ }^{8}$ Due to the close proximity to the obstacle the vertical component is negligible in this region.
} 
into the wrong direction as well as the average peak velocities of the correcting movements. These limits are likely to be induced by the setup.

Although implicitly not being allowed to stop in the via-point participants significantly slowed down in this area. One may wonder whether this slowdown provided them with sufficient time to process the presented location of the final movement target before making their decision where to move. The answer is a clear "no". Participants already make up their mind where to move way before reaching the via-point. ${ }^{9}$. The horizontal velocity in the intermediate target already serves as a reliable predictor for the chosen movement direction.

The possible strategies of choosing a movement direction when having no information about the future location can be rather diverse. i) Participants could extrapolate directly from the location of the previous trial - by just moving there again. ii) They also could try to predict the next target location based on a sequence of trials in the direct past. iii) They maybe could also try to estimate an overall frequency of occurrence and act accordingly. All of these hypotheses were tested on the data available from 16 probands ${ }^{10}$ who participated in four subsequent sessions (containing 250 successful trials each) both in their dominant and their non-dominant hand. On average over a session participants did not focus on the target location shown in the previous trial (cf. Section 6.4.1). It can be also shown that specific windows (or patches of history) of the series of target locations in direct past are unlikely to have been considered for the decision (cf. Section 6.4.2). Interestingly, subjects also did not seem to keep track of the overall presentation statistics, because otherwise their behaviour would have approximately matched the balanced presentation frequency (cf. Section 6.4.4). Instead, most of the participants showed a strong preference of one movement direction. This direction appeared to be independent of handedness and used hand but coincided with the initial bias of the experimental condition in case of initR. In this experimental condition all participants decided to take rightward movements as "default". However, in the experiments using the init $L$ condition not all participants picked up this initially suggested bias towards the left. Some of them decided to prefer the right, some of them the left, and a few showed a behaviour that approximately that was closer to matching the statistics of the presented target locations on the long run than to being biased to either side.

\footnotetext{
${ }^{9}$ Further attempts to trace the decision back in time - which means tracing it back in hand trajectory - are bound to fail due to the required high accuracy of hitting the viapoint. Directional preferences can only unfold in the trajectory in the very proximity of the intermediate target.

${ }^{10}$ Eight left-handed, eight right-handed - four of each handedness in each experimental
} 
Again this pattern seems to be independent of handedness and hand usage (cf. Section 6.4.1). Modelling attempts using presented target locations and individual choices of previous trials showed a great focus on what had been done in the past but neglected the importance of the presented locations (cf. Section 6.4.2). This constellation lead to the assumption of an internal nonlinear amplification of individual behavioural tendencies. During the first few trials initial "hints" on the location statistics are picked up in a more or less individual way - resulting in the preference of leftward movements in some, and rightward movements in other probands - and manifest in a decision default on the long run (cf. Section 6.3.3). A nonlinear approach with a bifurcation as its key element can be used to successfully model this increasing focus on perceived cues that turn into strong directional biases in the experimental data (cf. Section 6.4.4).

In contrast to the consistently emerging preference of always rightward movements in the initR condition, init $L$ not always lead to a preferences of leftward movements. This may result from the cultural imprinting of rightwards movements in reading, time lines, and also visual communication in our society. The learnt but probably often unconscious "default" expectation of the world being biased to the right strongly influences everyday decisions and may also play an important role in the context of motor decision. If the cues in the first tens of trials of a session match this expectation the default choice can be made without the need of further considerations. However, if the initial phase of a session shows a pattern that does not completely fit with the expectation further processing is required. Different outcomes in the level of biasedness in different participants may result from a number of mechanisms at this point. The individual degrees of attention to the distributions target locations is crucial for the detection of this initial imbalance in the stimuli. Furthermore, the individually perceived level of time pressure modulates the time that can be taken for further consideration of these cues. Taken together these factors give an idea about why some probands end up with the default choice even in case of initL while others follow the initially presented bias.

Interestingly, the individual averages of the horizontal velocity in the intermediate target not only show directional preferences but also reveal an interesting phenomenon about the sensitivity to the directional bias: the faster participants on average pass the via-point the stronger their final directional bias to one or the other side is. The algebraic sign of the average velocity furthermore indicates the direction of the bias (cf. Figure 6.3 in Section 6.4.1). This suggests that in cases of time pressure only a tiny patch of information 
about the environment is picked up and then amplified to serve as a decision heuristics, if the according costs for replanning are bearable.

In the given setup erroneous directional choices have to be corrected into a passing movement around the obstacle in the direction of the shortest way between the intermediate and the final movement target. The delay until this replanning act manifests as a deceleration in the horizontal component of hand velocity is on average highly consistent and similar across participants independent of handedness, hand usage or experimental condition. Processing of the information about the target location and initiating an according reaction to the discrepancy between planned and appropriate movement direction took about $257+/-28 \mathrm{~ms}$. This delay matches average delays known from as the "autopilot" threshold (cf. Pisella et al., 2000), which represents the earliest time when a reaction to a target jump is detectable in hand trajectory. Also the timing of the peak of the correcting movement is relatively constant across participants (independent off their movement speed!) at about $712+/-94$ ms. Furthermore, a linear relation between peak velocity of the excursion into the wrong direction and peak velocity of the correction could be found (cf. Section 5.4.2).

In addition to the setup-induced limits to peak velocities in cases of direct and also replanned movements as well as the regular timing of the replanning even when compared across participants this is consistent with the assumption of movement primitives for according submovements. For a detailed analysis of the replanning process of erroneous movements I used symmetric bell-shaped velocity profiles with three degrees of freedom ${ }^{11}$ (cf. Plamondon et al., 1993), which are applicable for the quasi-straight movement segments between viapoint and the passage of the horizontal centre line of the obstacle. First I derived the average movement primitives for direct movements to the left and the right of single sessions of individual participants. Then I modelled replanned movements based on the direct movement into the wrong direction - that is smoothly faded out after a reaction time needed to process the new stimulus - and on another correcting primitive towards the right direction. This superposition lead to earlier replanning times in the range of (and even earlier than) the earliest auto-pilot thresholds of $120 \mathrm{~ms}$. These replanning times reflect the onset of the replanning before it results in detectable changes in hand trajectory.

In the second part of my thesis I presented a two-alternative forced-choice task to study the interacting factors during the complex process of human mo-

${ }^{11}$ Start and end time of the primitive, and peak velocity. 
tor decisions under time pressure. Although handedness and hand usage play an important role in everyday life their effects on the decision process in the presented task did not significantly influence the probands behaviour. Due to the limited time granted for processing of the according stimuli, also direct observations of the presented scene only played a minor role. Instead, mostly unconscious initial ideas about the distribution of target locations - which might result in a vague intuition at most - and the amplification of the individual behavioural pattern in response to these ideas were shown to be the main factors in the decision process. Under time pressure participants on average tended to quickly repeat their own behaviour and, thus, showed stereotypical choices of movement directions. The heuristic, which can be derived from this decision behaviour, contains a significant preference of the culturally imprinted direction of movements. A first guess about the environmental statistics can serve as a basis for initial decisions. The preference of these directional decisions practically: the frequency of choosing them instead of other alternatives - then can increased over time. By using this type of default bahviour the problem of the often too time-consuming decision process, which would have to be carried out in each decision situation again, can be changed into the derivation of a sufficiently good initial guess about the presented environment. Resulting behaviour probably would seem "somehow natural" to human observers and, hence, increase acceptance of robots in everyday life. 


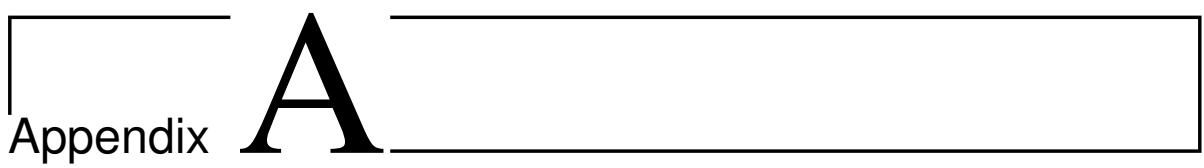

\section{Appendix}

\section{A.1 Supplementary material}

A.1.1 Overview of behavioral regularities in subsequent trials

A.1.2 Full compilation of sessionwise ARMA error plots

A.1.3 Kink count tables

A.1.4 Error correction in terms of bell-shaped velocity profiles 
Table A.1: Kink counts of initR condition, split by handedness, hand usage, and session. $\mathrm{K} 2 \mathrm{~L}=$ kink to the left, $\mathrm{K} 2 \mathrm{R}=$ kink to the right.

\begin{tabular}{|c|c|c|c|c|c|c|c|c|}
\hline \multicolumn{9}{|c|}{ Right-handed } \\
\hline \multicolumn{9}{|c|}{ Used right hand } \\
\hline Subj. & \multicolumn{4}{|c|}{ CK } & \multicolumn{4}{|c|}{$\mathrm{EE}$} \\
\hline Sess. & 1 & 2 & 3 & 4 & 1 & 2 & 3 & 4 \\
\hline K2R & 89 & 111 & 113 & 110 & 67 & 91 & 87 & 95 \\
\hline $\mathrm{K} 2 \mathrm{~L}$ & 10 & 4 & 0 & 0 & 13 & 10 & 8 & 10 \\
\hline \multicolumn{9}{|c|}{ Used left hand } \\
\hline $\mathrm{K} 2 \mathrm{R}$ & 61 & 79 & 108 & 114 & 90 & 100 & 101 & 104 \\
\hline $\mathrm{K} 2 \mathrm{~L}$ & 38 & 28 & 9 & 0 & 5 & 6 & 4 & 4 \\
\hline \multicolumn{9}{|c|}{ "Right-handed } \\
\hline \multicolumn{9}{|c|}{ Used right hand } \\
\hline Subj. & \multicolumn{4}{|c|}{ DB } & \multicolumn{4}{|c|}{$\mathrm{KF}$} \\
\hline Sess. & 1 & 2 & 3 & 4 & 1 & 2 & 3 & 4 \\
\hline K2R & 85 & 100 & 104 & 104 & 94 & 101 & 105 & 94 \\
\hline $\mathrm{K} 2 \mathrm{~L}$ & 13 & 14 & 2 & 2 & 15 & 8 & 7 & 14 \\
\hline \multicolumn{9}{|c|}{ Used left hand } \\
\hline \multirow[t]{2}{*}{$\overline{\mathrm{K} 2 \mathrm{R}}$} & 61 & 79 & 108 & 114 & 90 & 100 & 101 & 104 \\
\hline & 106 & 111 & 111 & 108 & 45 & 42 & 58 & 55 \\
\hline $\mathrm{K} 2 \mathrm{~L}$ & 38 & 28 & 9 & 0 & 5 & 6 & 4 & 4 \\
\hline \multicolumn{9}{|c|}{ "Left-handed } \\
\hline \multicolumn{9}{|c|}{ Used left hand } \\
\hline Subj. & \multicolumn{4}{|c|}{ BK } & \multicolumn{4}{|c|}{ SA } \\
\hline Sess. & 1 & 2 & 3 & 4 & 1 & 2 & 3 & 4 \\
\hline K2R & 79 & 95 & 95 & 94 & 32 & 53 & 94 & 103 \\
\hline $\mathrm{K} 2 \mathrm{~L}$ & 9 & 3 & 4 & 7 & 52 & 57 & 7 & 7 \\
\hline \multicolumn{9}{|c|}{ Used right hand } \\
\hline K2R & 93 & 95 & 103 & 98 & 92 & 109 & 114 & 104 \\
\hline $\mathrm{K} 2 \mathrm{~L}$ & 11 & 8 & 2 & 8 & 12 & 7 & 1 & 7 \\
\hline \multicolumn{9}{|c|}{ Left-handed } \\
\hline \multicolumn{9}{|c|}{ Used left hand } \\
\hline Subj. & \multicolumn{4}{|c|}{ MT } & \multicolumn{4}{|c|}{ JS } \\
\hline Sess. & 1 & 2 & 3 & 4 & 1 & 2 & 3 & 4 \\
\hline K2R & 57 & 46 & 32 & 33 & 64 & 65 & 81 & 91 \\
\hline $\mathrm{K} 2 \mathrm{~L}$ & 37 & 61 & 81 & 87 & 34 & 29 & 22 & 13 \\
\hline \multicolumn{9}{|c|}{ Used right hand } \\
\hline K2R & 95 & 112 & 111 & 108 & 95 & 112 & 111 & 108 \\
\hline $\mathrm{K} 2 \mathrm{~L}$ & 13 & 3 & 0 & 2 & 13 & 3 & 0 & 2 \\
\hline
\end{tabular}


Table A.2: Kink counts of initL condition, split by handedness, hand usage, and session. $\mathrm{K} 2 \mathrm{~L}=$ kink to the left, $\mathrm{K} 2 \mathrm{R}=$ kink to the right.

\begin{tabular}{|c|c|c|c|c|c|c|c|c|}
\hline \multicolumn{9}{|c|}{ Right-handed } \\
\hline \multicolumn{9}{|c|}{ Used right hand } \\
\hline Subj. & \multicolumn{4}{|c|}{ DL } & \multicolumn{4}{|c|}{ KW } \\
\hline Sess. & 1 & 2 & 3 & 4 & 1 & 2 & 3 & 4 \\
\hline $\mathrm{K} 2 \mathrm{R}$ & 37 & 34 & 23 & 13 & 53 & 9 & 16 & 15 \\
\hline $\mathrm{K} 2 \mathrm{~L}$ & 75 & 89 & 91 & 93 & 73 & 99 & 89 & 88 \\
\hline \multicolumn{9}{|c|}{ Used left hand } \\
\hline $\mathrm{K} 2 \mathrm{R}$ & 17 & 5 & 9 & 15 & 78 & 114 & 110 & 109 \\
\hline $\mathrm{K} 2 \mathrm{~L}$ & 85 & 97 & 100 & 99 & 25 & 1 & 6 & 4 \\
\hline \multicolumn{9}{|c|}{ Right-handed } \\
\hline \multicolumn{9}{|c|}{ Used right hand } \\
\hline Subj. & \multicolumn{4}{|c|}{ JA } & \multicolumn{4}{|c|}{$\mathrm{MB}$} \\
\hline Sess. & 1 & 2 & 3 & 4 & 1 & 2 & 3 & 4 \\
\hline $\mathrm{K} 2 \mathrm{R}$ & 49 & 56 & 74 & 93 & 37 & 9 & 7 & 18 \\
\hline $\mathrm{K} 2 \mathrm{~L}$ & 39 & 36 & 32 & 13 & 69 & 96 & 101 & 96 \\
\hline \multicolumn{9}{|c|}{ Used left hand } \\
\hline $\mathrm{K} 2 \mathrm{R}$ & 70 & 102 & 112 & 115 & 4 & 1 & 3 & 1 \\
\hline $\mathrm{K} 2 \mathrm{~L}$ & 35 & 10 & 5 & 1 & 104 & 107 & 109 & 111 \\
\hline
\end{tabular}

\begin{tabular}{|l|c|c|c|c||c|c|c|c|}
\hline \multicolumn{10}{|c|}{ Left-handed } \\
\hline \multicolumn{10}{|c|}{ UK2 left hand } \\
\hline Subj. & \multicolumn{1}{|c|}{ Used right hand } \\
\hline Sess. & 1 & 2 & 3 & 4 & 1 & 2 & 3 & 4 \\
\hline K2R & 46 & 56 & 64 & 68 & 34 & 44 & 101 & 102 \\
\hline K2L & 52 & 49 & 49 & 35 & 63 & 75 & 17 & 14 \\
\hline \hline \multicolumn{10}{|c|}{} & Und \\
\hline K2R & 70 & 95 & 96 & 92 & 90 & 88 & 38 & 41 \\
\hline K2L & 37 & 16 & 12 & 29 & 20 & 31 & 58 & 77 \\
\hline
\end{tabular}

\begin{tabular}{|l|c|c|c|c||c|c|c|c|}
\hline \multicolumn{10}{|c|}{ Ueft-handed } \\
\hline \multicolumn{10}{|c|}{ Uwed left hand } \\
\hline Subj. & \multicolumn{1}{|c|}{ Used right hand } \\
\hline Sess. & 1 & 2 & 3 & 4 & 1 & 2 & 3 & 4 \\
\hline K2R & 19 & 18 & 13 & 23 & 62 & 96 & 77 & 82 \\
\hline K2L & 89 & 98 & 96 & 82 & 49 & 23 & 24 & 37 \\
\hline \hline \multicolumn{10}{|c|}{} & \multicolumn{10}{|c|}{ YB } \\
\hline K2R & 93 & 107 & 113 & 105 & 60 & 25 & 11 & 11 \\
\hline K2L & 22 & 5 & 6 & 11 & 42 & 81 & 94 & 100 \\
\hline
\end{tabular}




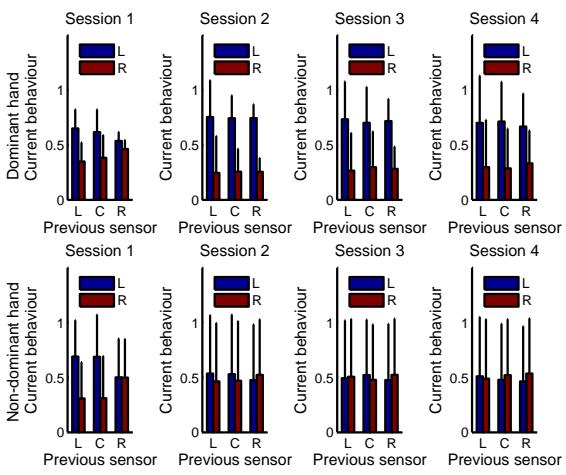

(a) Right-handed, condition initL.

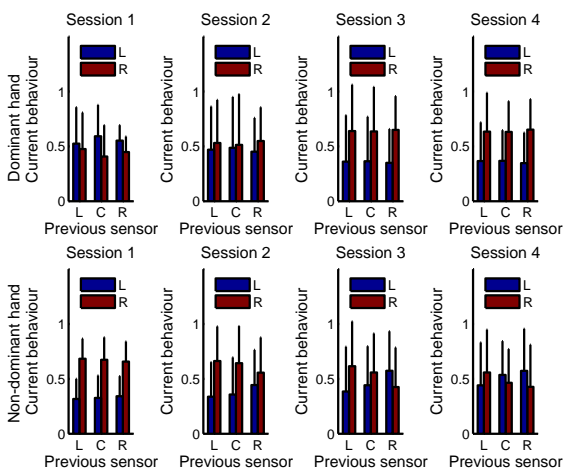

(c) Left-handed, condition initL.

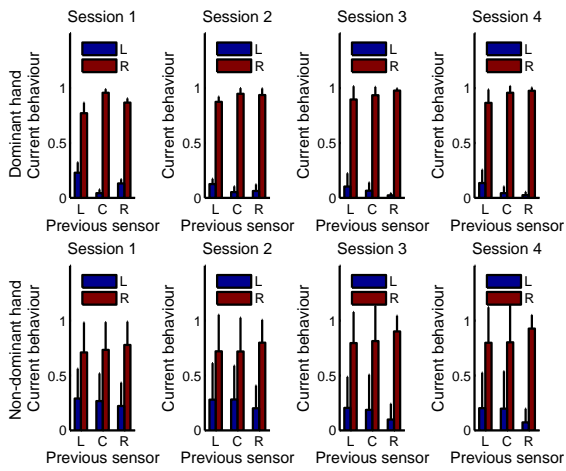

(b) Right-handed, condition initR.
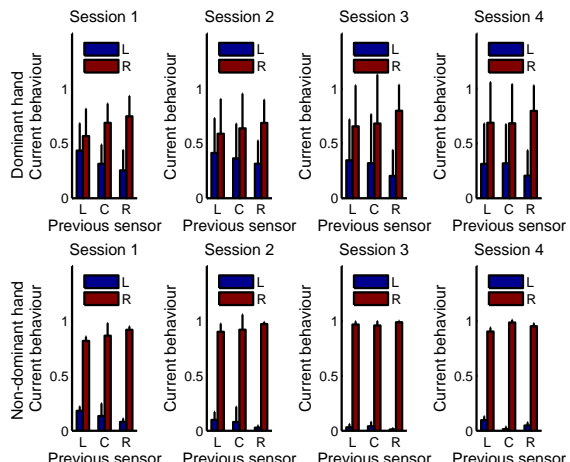

(d) Left-handed, condition initR.

Figure A.1: Influence of previously perceived target T2 location on directional choices in subsequent trials. Normalisation done within group of trials the same previous location of the final movement target. Plots show fraction of trials that went to the left (blue bars) and to the right (red bars) to all trials with the given previously shown location of target T2. Upper rows of subfigures contain data from dominant hand, lower rows from non-dominant hand. 


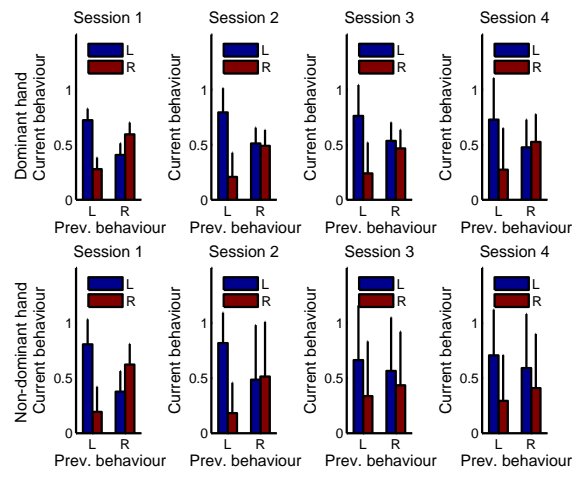

(a) Right-handed, condition initL.

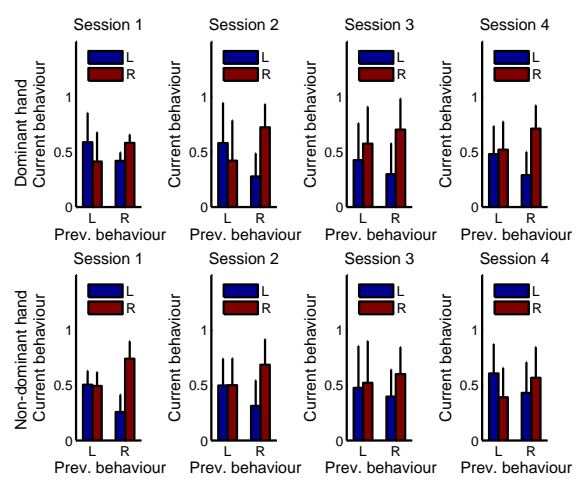

(c) Left-handed, condition initL.

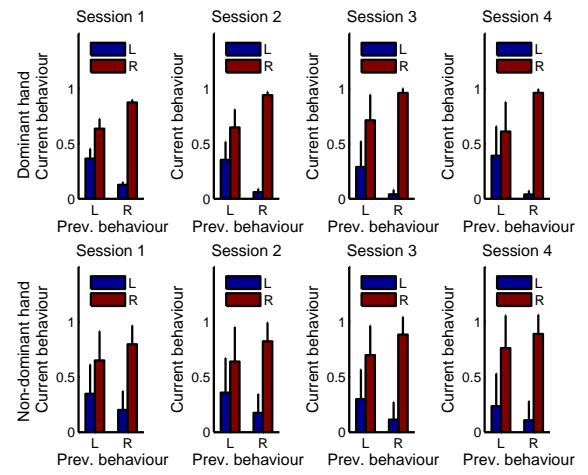

(b) Right-handed, condition initR.

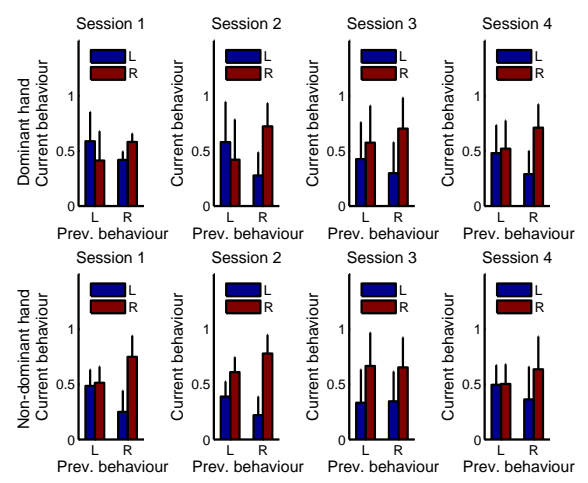

(d) Left-handed, condition initR.

Figure A.2: Influence of previously chosen movement direction on directional choices in subsequent trials. Normalisation done within group of trials the same previous choice of movement direction. Plots show fraction of trials that went to the left (blue bars) and to the right (red bars) to all trials with the given previous behaviour. Upper rows of subfigures contain data from dominant hand, lower rows from non-dominant hand. Numbers of participants same as in Figure A.1 


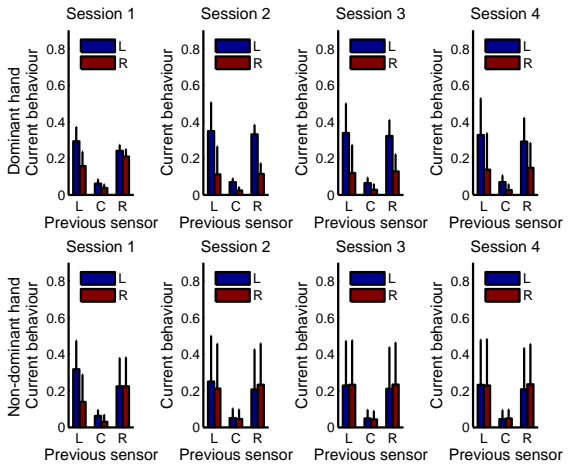

(a) Right-handed, condition initL.

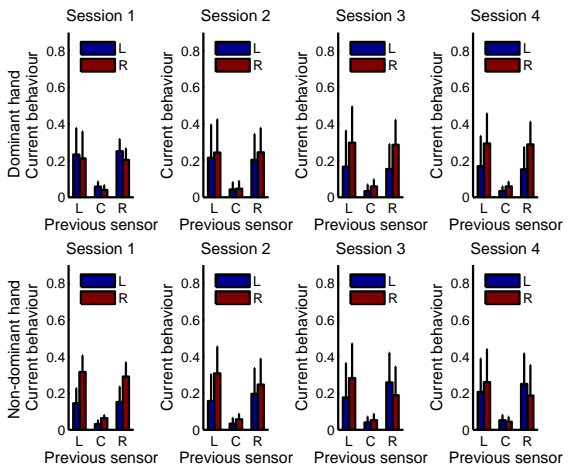

(c) Left-handed, condition initL.

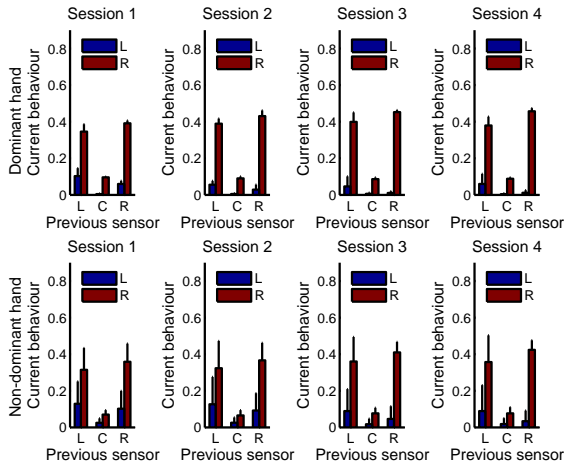

(b) Right-handed, condition initR.
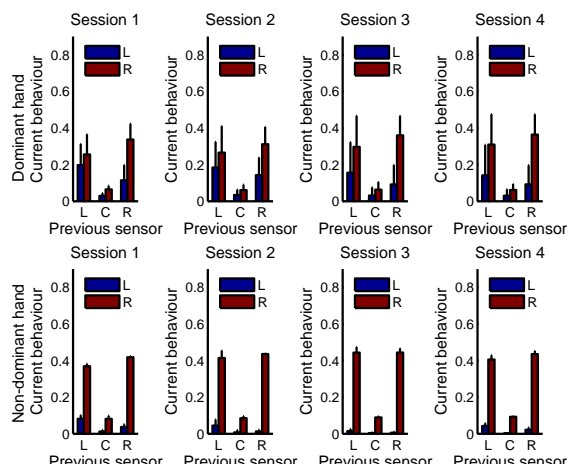

(d) Left-handed, condition initR.

Figure A.3: Influence of previously perceived target T2 location on directional choices in subsequent trials. Normalisation done with respect to all trials. Plots show fraction of trials that went to the left (blue bars) and to the right (red bars) to all trials with the given previously shown location of target T2. Upper rows of subfigures contain data from dominant hand, lower rows from non-dominant hand. 


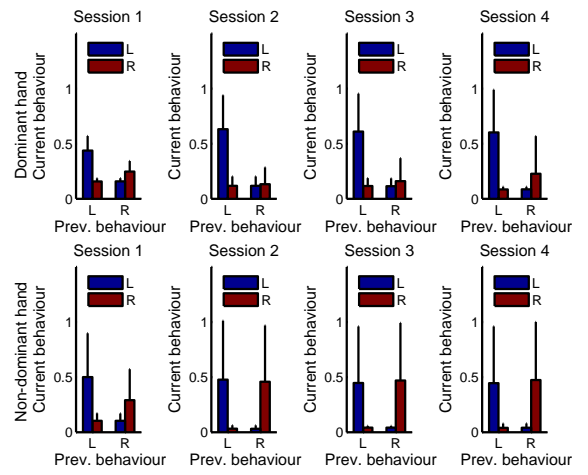

(a) Right-handed, condition initL.

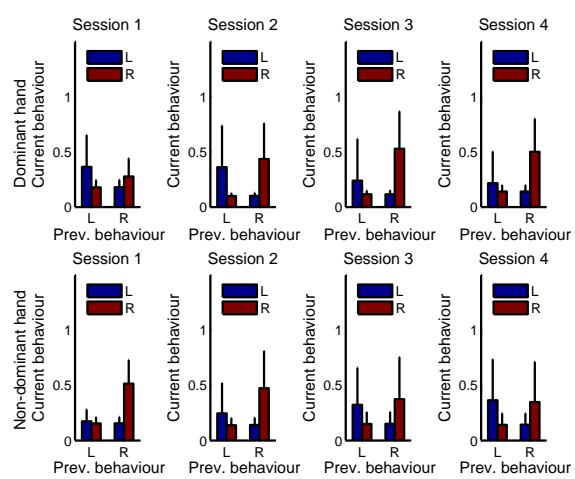

(c) Left-handed, condition initL.

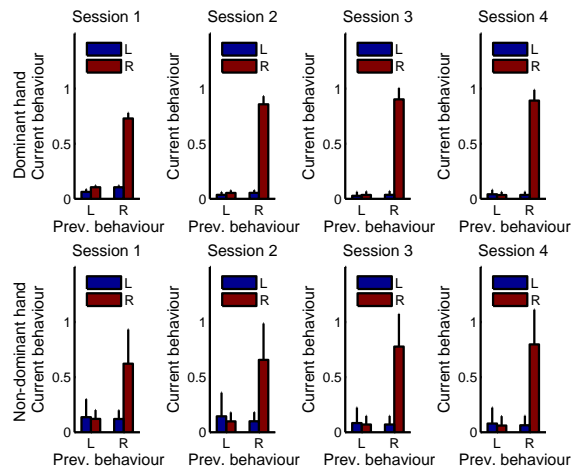

(b) Right-handed, condition initR.

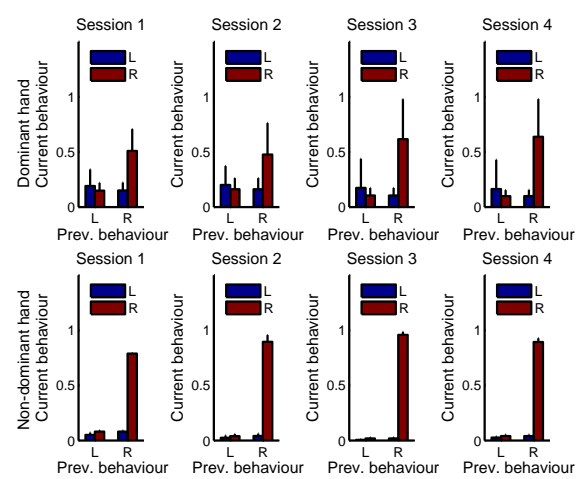

(d) Left-handed, condition initR.

Figure A.4: Influence of previously chosen movement direction on directional choices in subsequent trials. Normalisation done with respect to all trials. Plots show fraction of trials that went to the left (blue bars) and to the right (red bars) to all trials with the given previous behaviour. Upper rows of subfigures contain data from dominant hand, lower rows from non-dominant hand. 

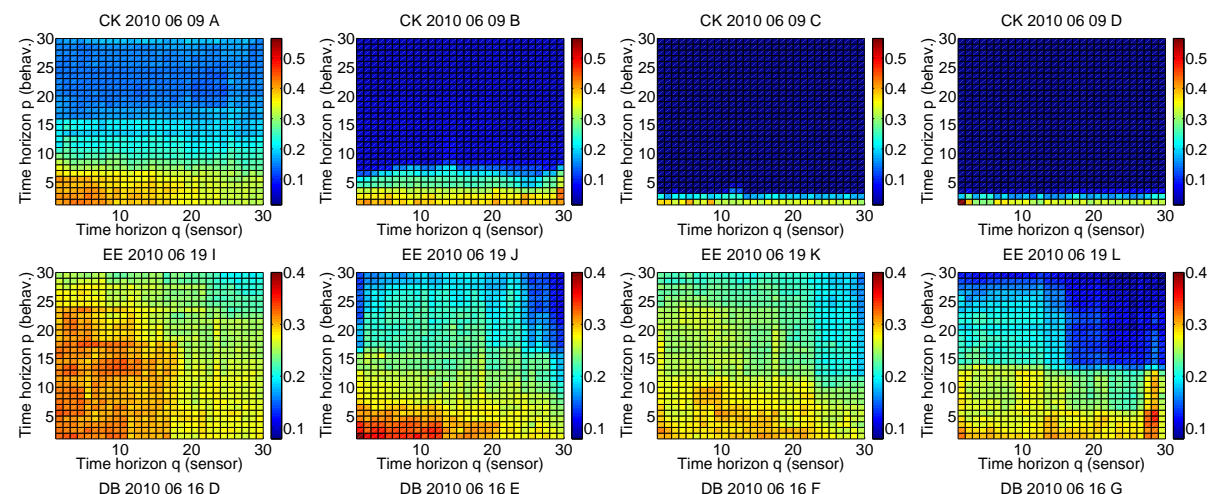

EE 20100619 L

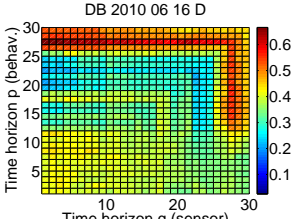

DB $20100616 \mathrm{E}$

DB $20100616 \mathrm{~F}$

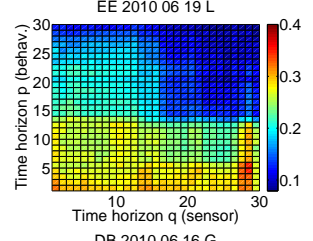

KF 20100607 A
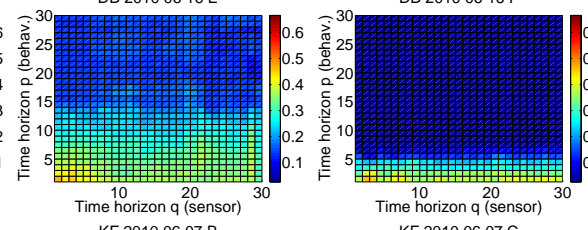

DB $20100616 \mathrm{G}$
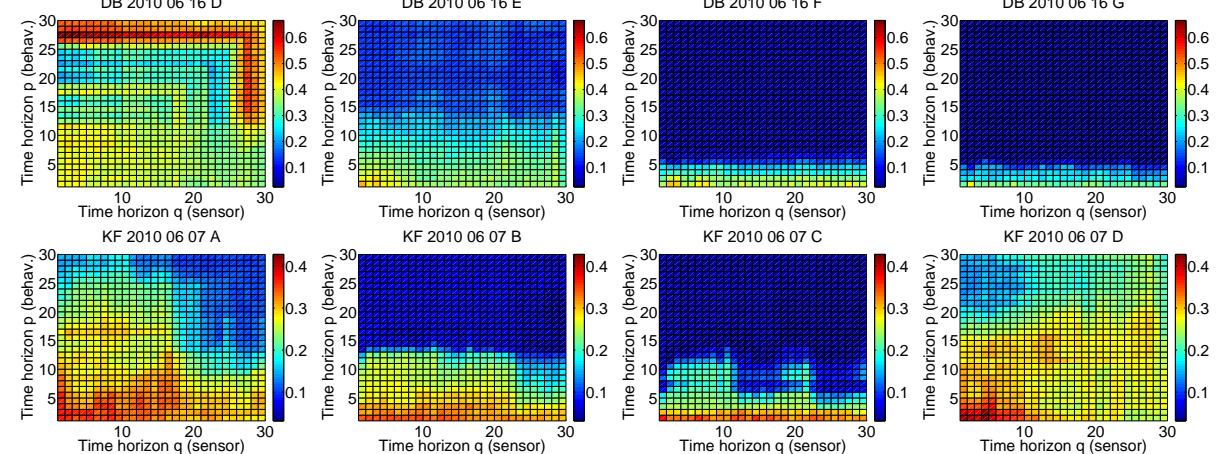

Figure A.5: Prediction errors for right-handed participants using dominant hand (biased to the right): Summed differences for behaviour prediction in dependence on time horizons $p$ in presented directions and $q$ in own behaviour. 


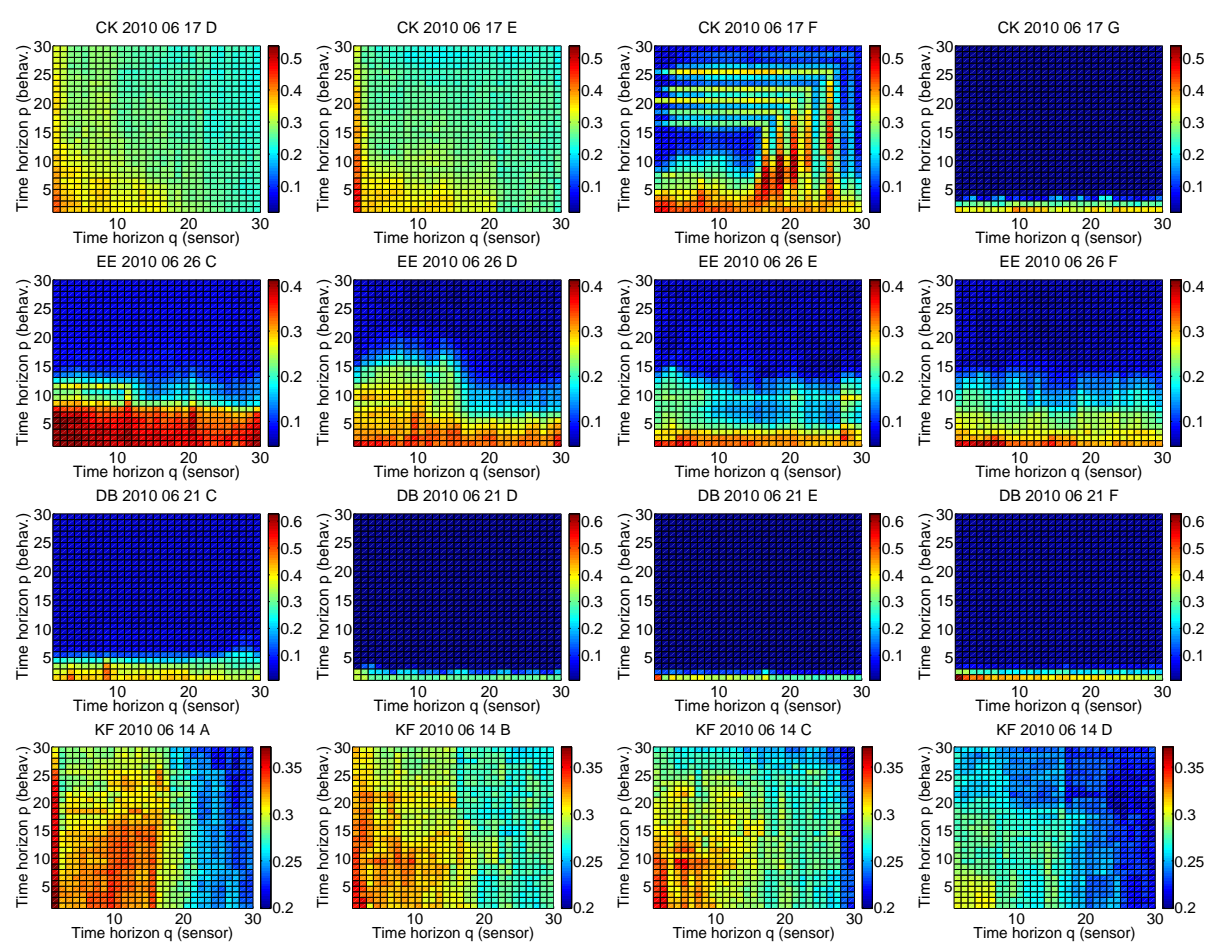

Figure A.6: Prediction errors for right-handed participants using non-dominant hand (biased to the right): Summed differences for behaviour prediction in dependence on time horizons $p$ in presented directions and $q$ in own behaviour. 


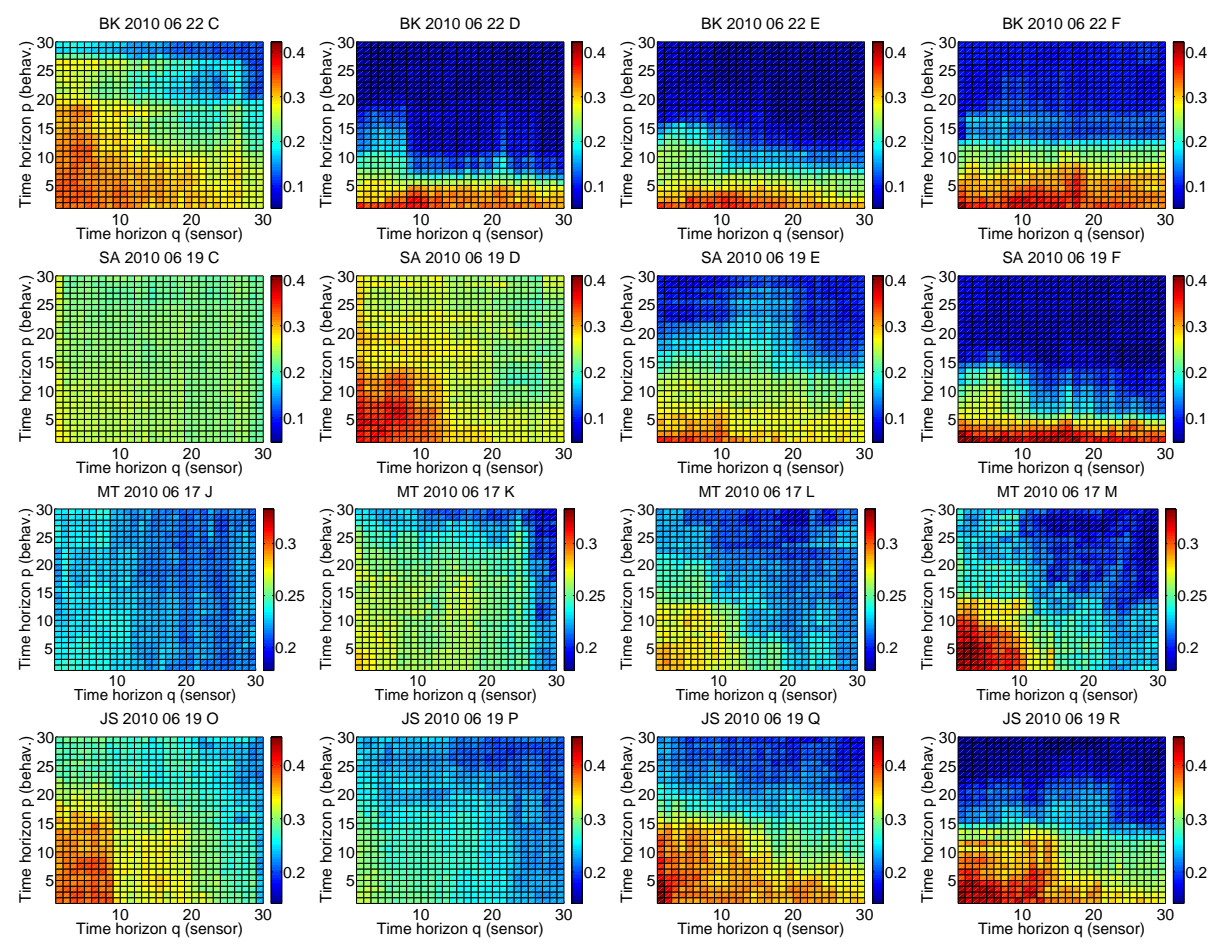

Figure A.7: Prediction errors for left-handed participants using their dominant hand (biased to the right): Summed differences for behaviour prediction in dependence on time horizons $p$ in presented directions and $q$ in own behaviour. 


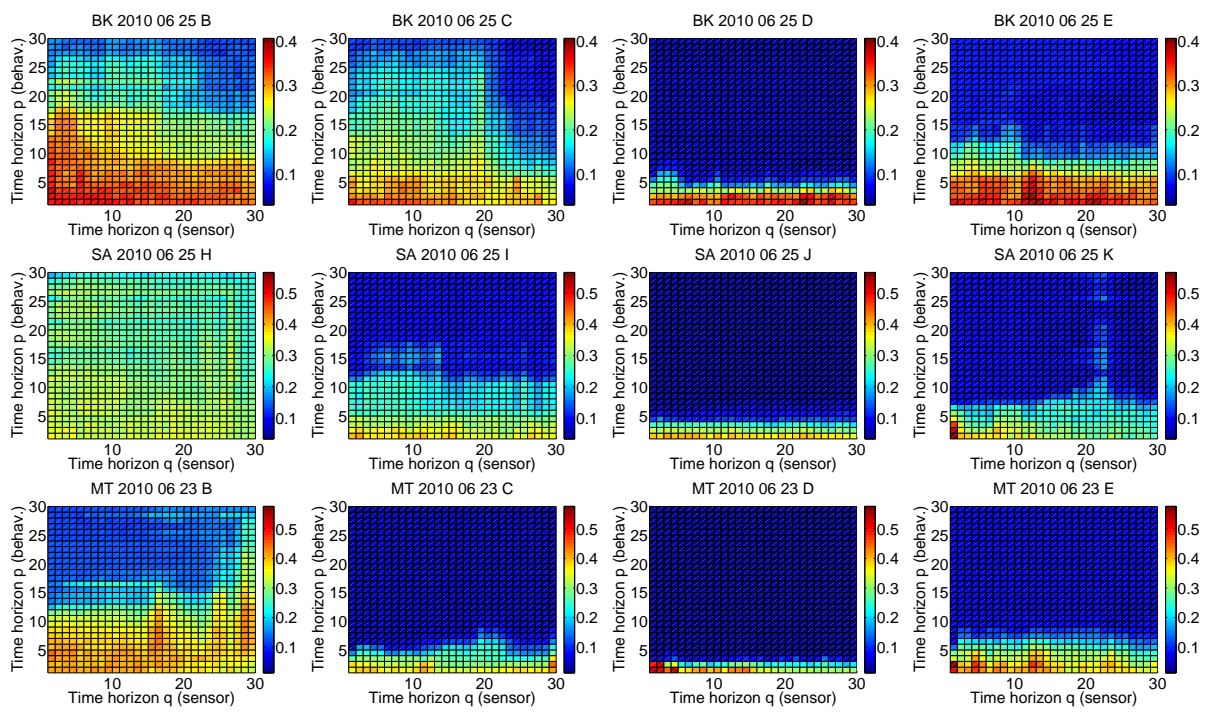

Figure A.8: Prediction errors for left-handed participants using their non-dominant hand (biased to the right): Summed differences for behaviour prediction in dependence on time horizons $p$ in presented directions and $q$ in own behaviour. 

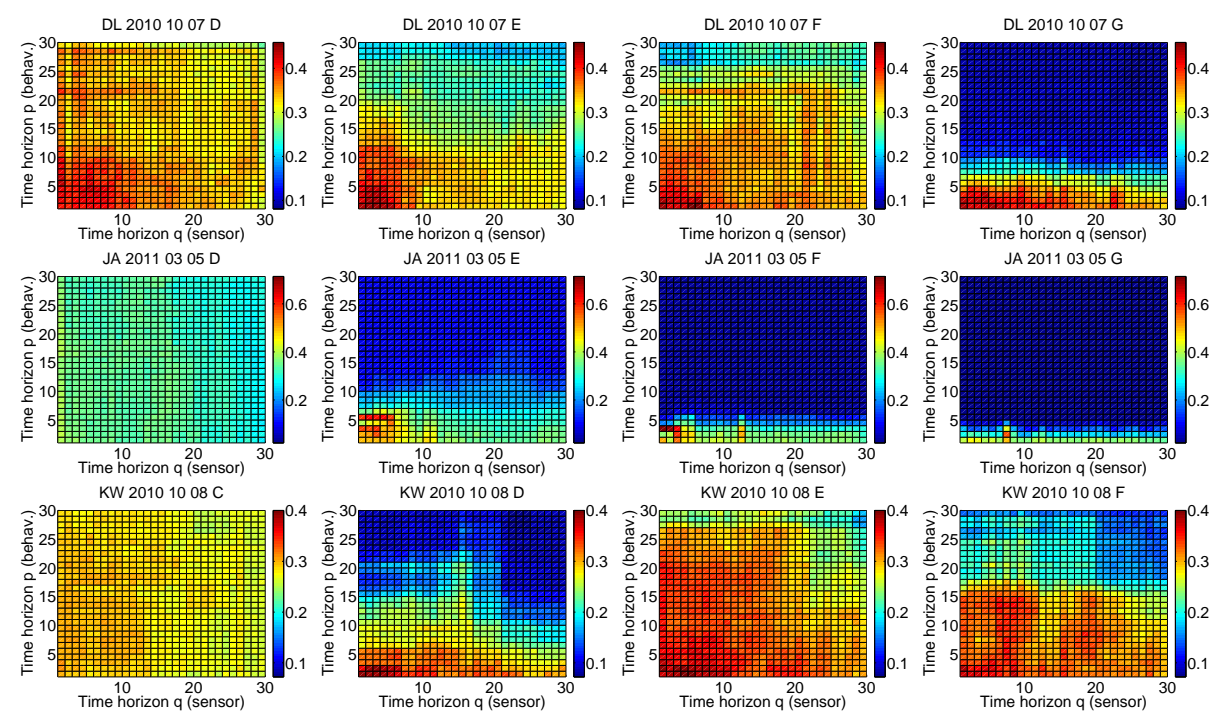

KW 20101008 D
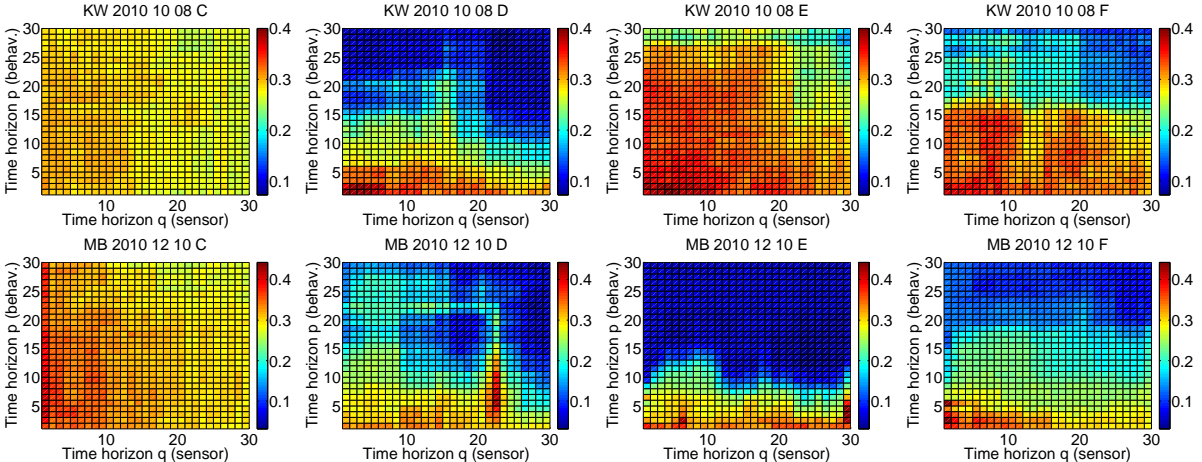

Figure A.9: Prediction errors for right-handed participants using dominant hand (biased to the left): Summed differences for behaviour prediction in dependence on time horizons $p$ in presented directions and $q$ in own behaviour. 
DL 20101011 I
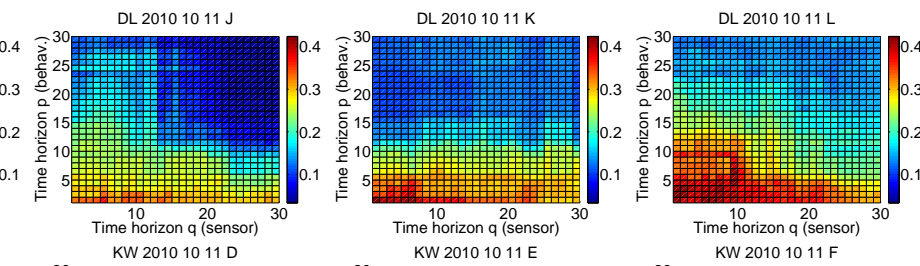

KW 20101011 C
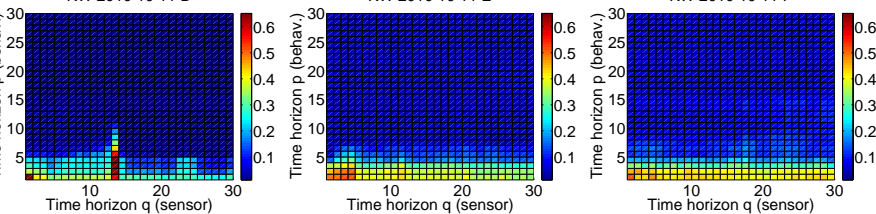

MB 20101217 C MB 20101217 D MB $20101217 \mathrm{E}$ MB $20101217 \mathrm{~F}$
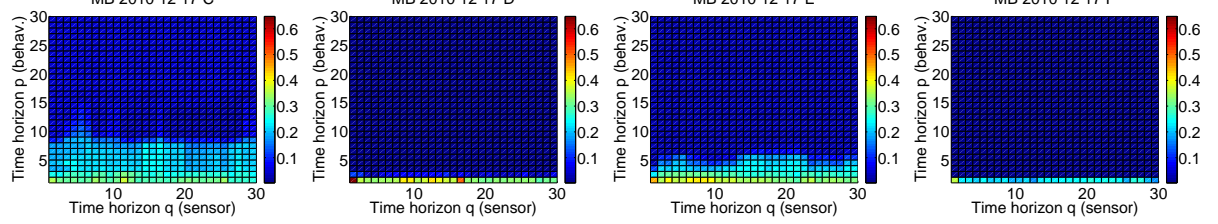

Figure A.10: Prediction errors for right-handed participants using non-dominant hand (biased to the left): Summed differences for behaviour prediction in dependence on time horizons $p$ in presented directions and $q$ in own behaviour. 


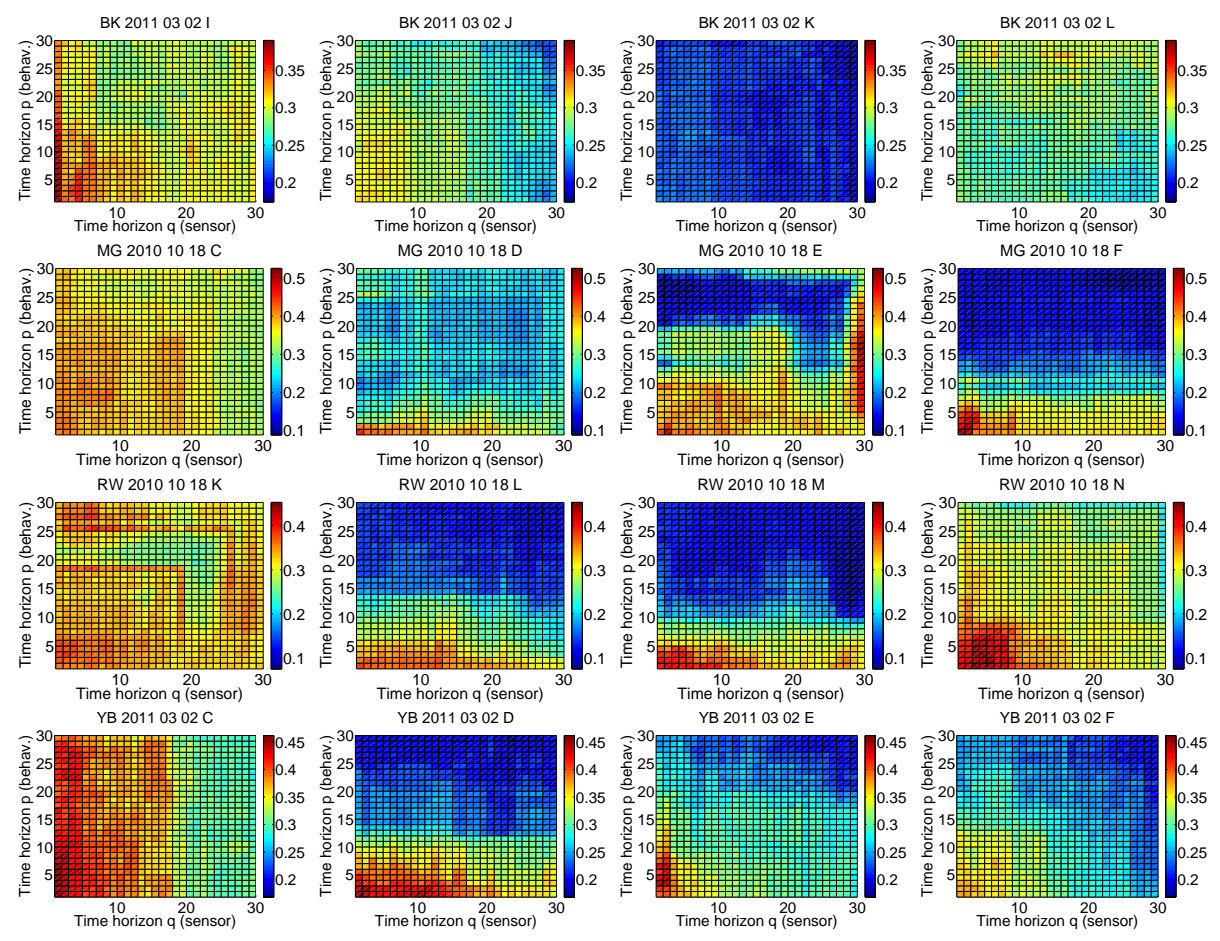

Figure A.11: Prediction errors for left-handed participants using their dominant hand (biased to the left): Summed differences for behaviour prediction in dependence on time horizons $p$ in presented directions and $q$ in own behaviour. 


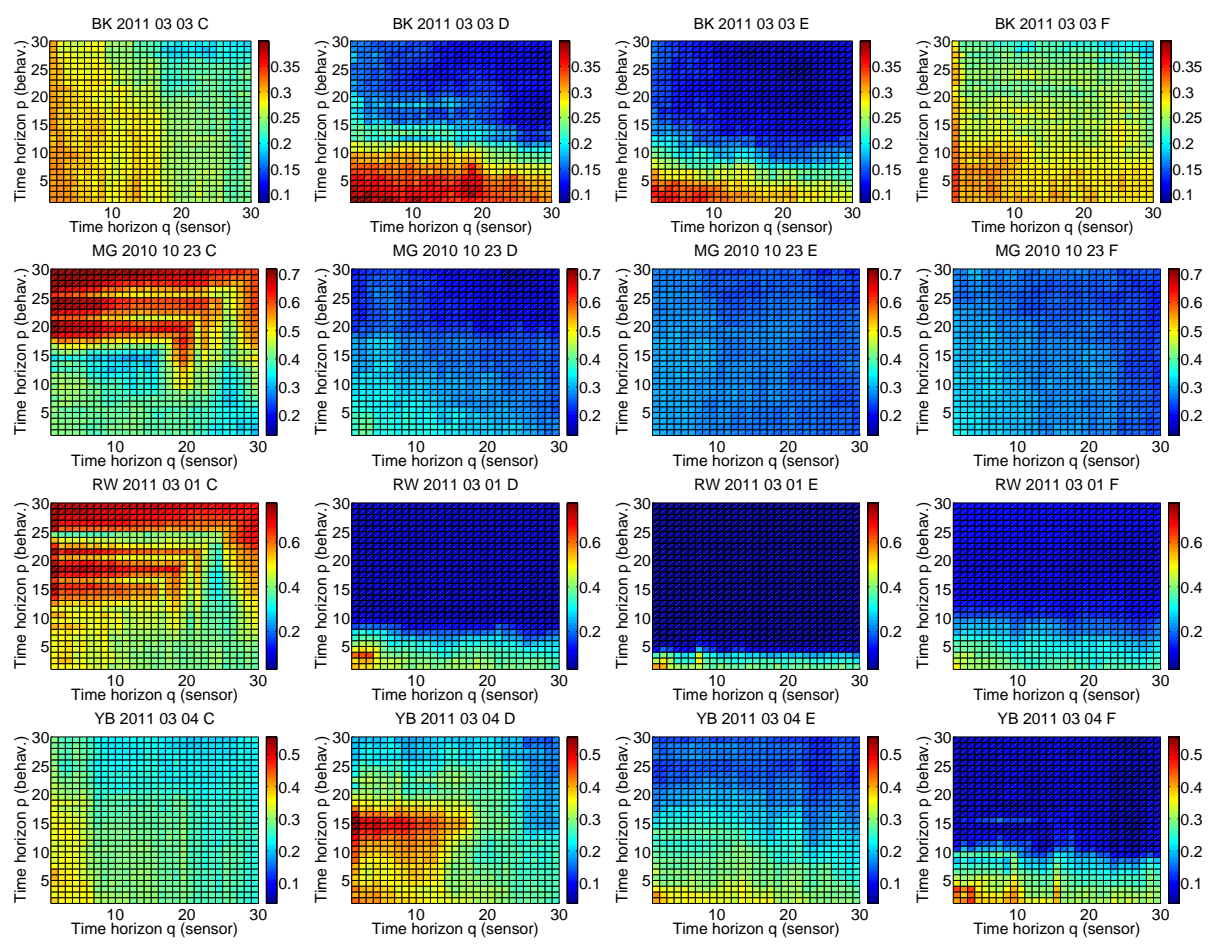

Figure A.12: Prediction errors for left-handed participants using their non-dominant hand (biased to the left): Summed differences for behaviour prediction in dependence on time horizons $p$ in presented directions and $q$ in own behaviour. (BK2, MG, RW, YB 
CK - Dominant hand (biasR)
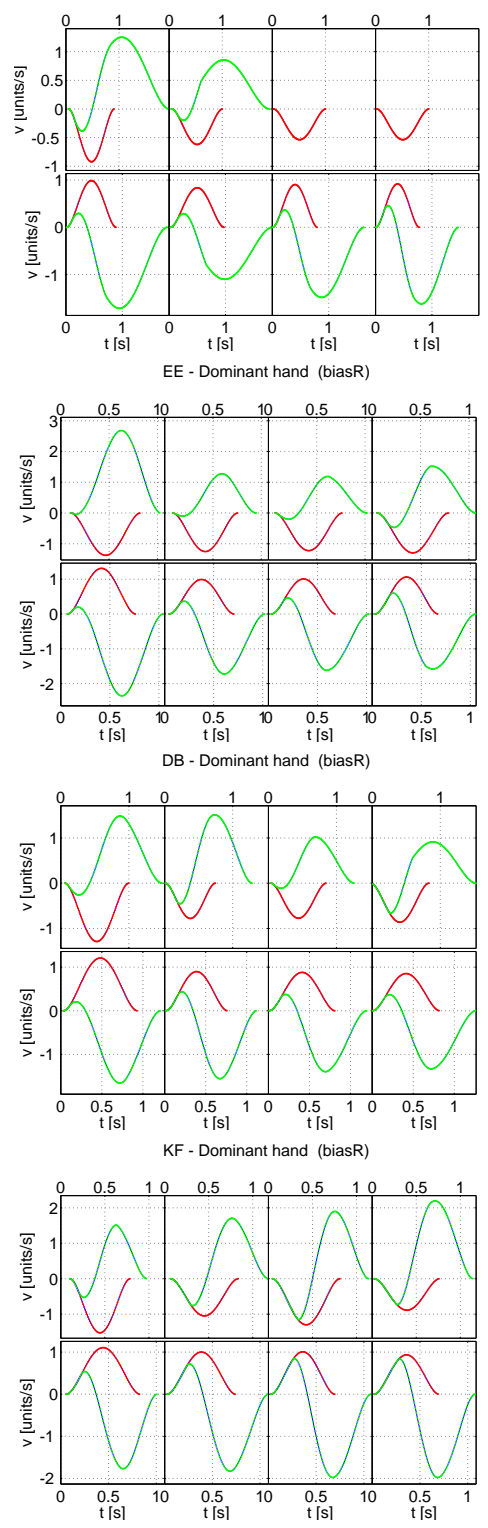

CK - Non-dominant hand (biasR)
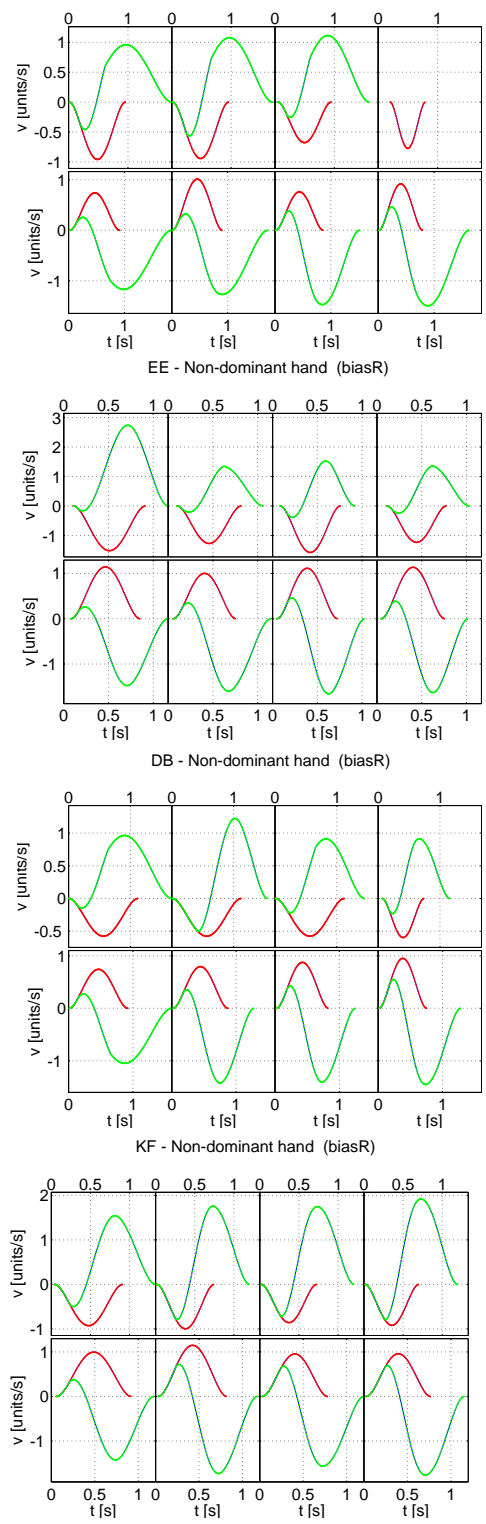

Figure A.13: Average velocity profiles of direct movements (red) and corrected ones (green) generated by adaptive bell fits for $v_{x}(t)$ between arrival in target $\mathrm{T} 1\left(t_{T 1}\right)$ and wall pass $\left(T_{W}\right)$ for right-handed participants in experimental condition of bias to the right. Left column contains data from dominant hand, right column from the non-dominant one. Upper row of each subfigure contains kinks to the left, lower row kinks to the right, respectively. Columns of the subfigures indicate sessions. Kinks to the left have to be understood cum grano due to their sparseness in this experimental condition (see Section 6.4.1). No green graph means that there were no according movements. (CK, EE, DB, KF) 

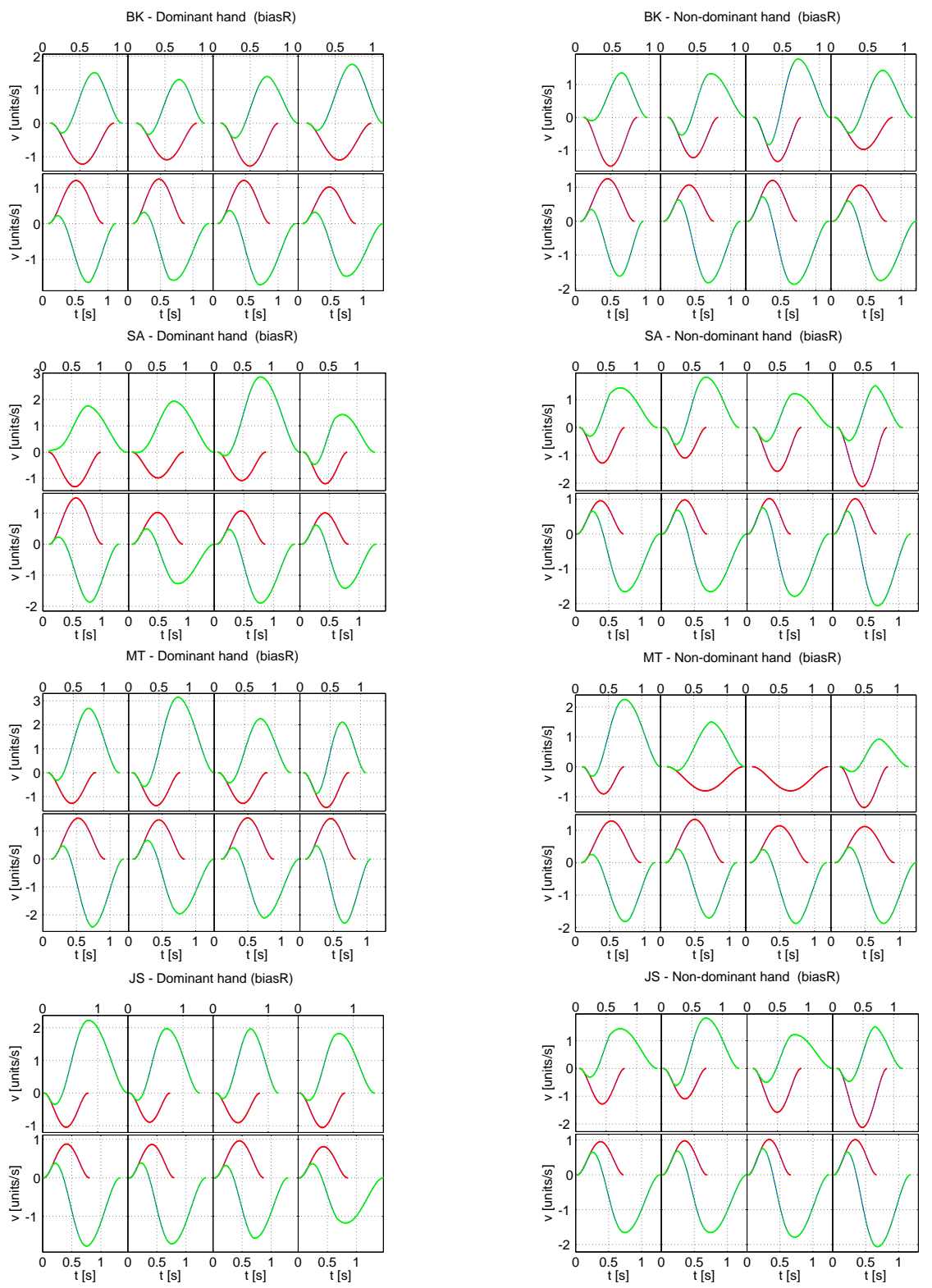

Figure A.14: Average velocity profiles of direct movements (red) and corrected ones (green) generated by adaptive bell fits for $v_{x}(t)$ between arrival in target $\mathrm{T} 1\left(t_{T 1}\right)$ and wall pass $\left(T_{W}\right)$ for left-handed participants in experimental condition of bias to the right. Left column contains data from dominant hand, right column from the non-dominant one. Upper row of each subfigure contains kinks to the left, lower row kinks to the right, respectively. Columns of the subfigures indicate sessions. Kinks to the left have to be understood cum grano due to their sparseness in this experimental condition (see Section 6.4.1). No green graph means that there were no according movements. (BK, SA, MT, JS) 

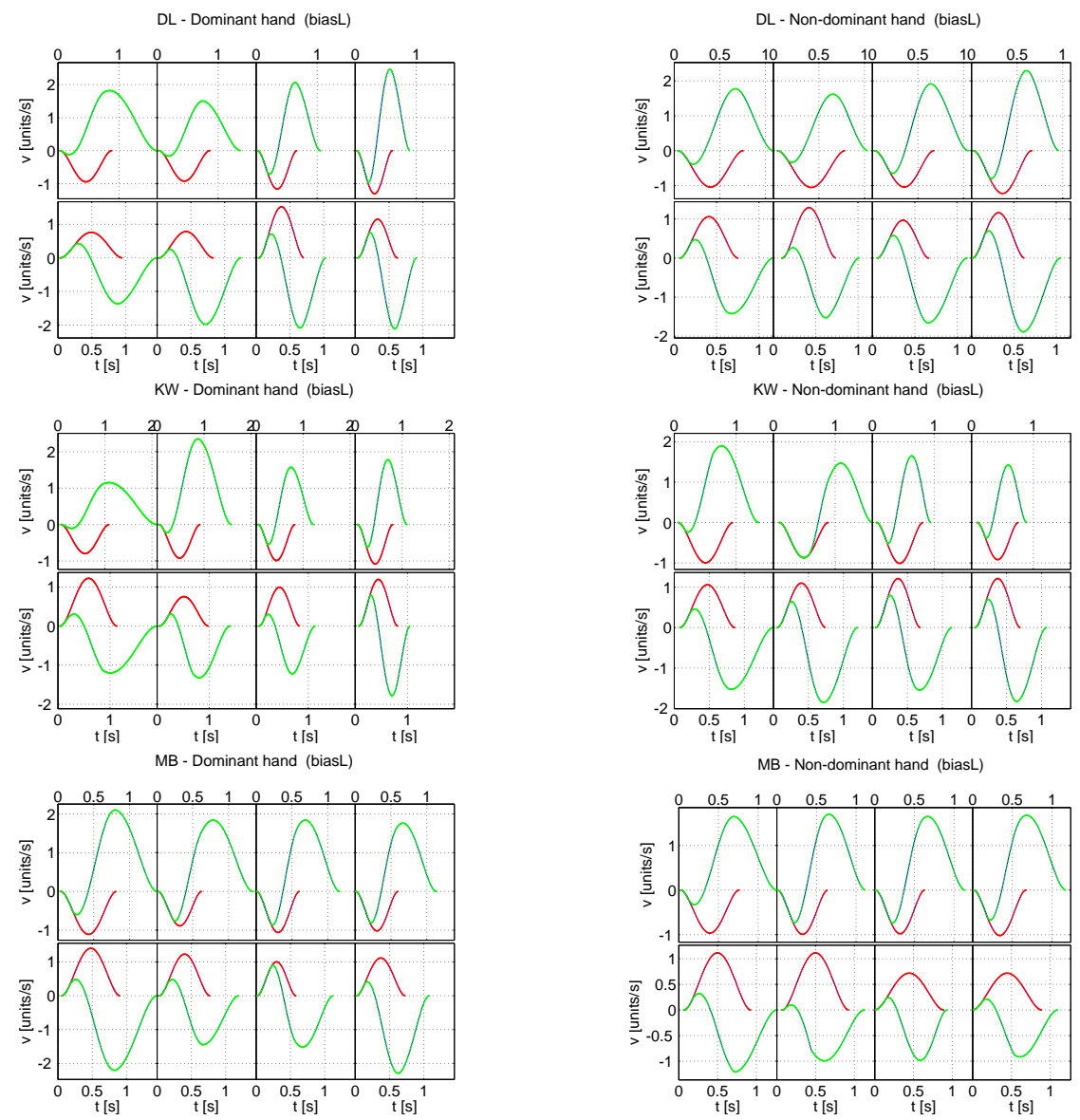

Figure A.15: Average velocity profiles of direct movements (red) and corrected ones (green) generated by adaptive bell fits for $v_{x}(t)$ between arrival in target T1 $\left(t_{T 1}\right)$ and wall pass $\left(T_{W}\right)$ for right-handed participants in experimental condition of bias to the left. Left column contains data from dominant hand, right column from the non-dominant one. Upper row of each subfigure contains kinks to the left, lower row kinks to the right, respectively. Columns of the subfigures indicate sessions. Kinks to the right have to be understood cum grano due to their sparseness in this experimental condition (see Section 6.4.1). (DL, KW, JA) 
MG - Dominant hand (biasL)
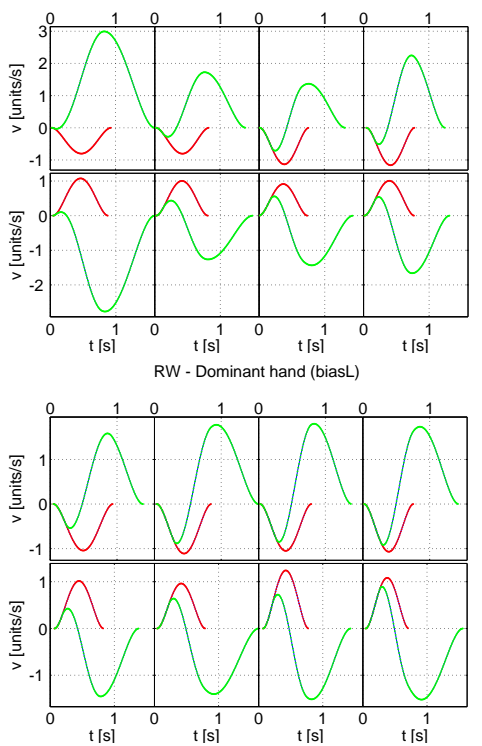

MG - Non-dominant hand (biasL)

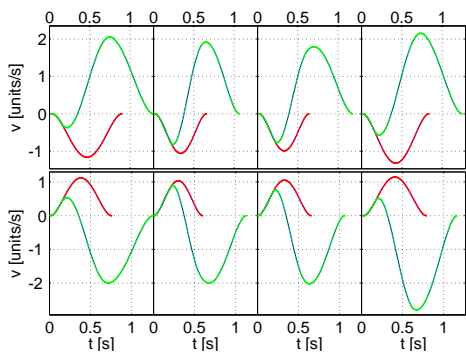

RW - Non-dominant hand (biasL)

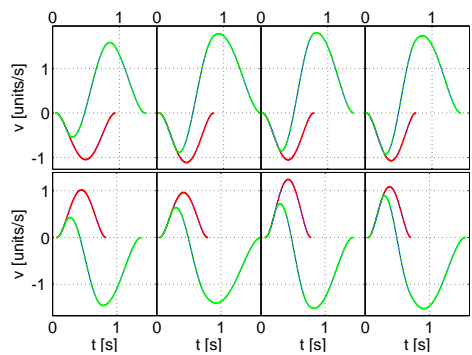

Figure A.16: Average velocity profiles of direct movements (red) and corrected ones (green) generated by adaptive bell fits for $v_{x}(t)$ between arrival in target $\mathrm{T} 1\left(t_{T 1}\right)$ and wall pass $\left(T_{W}\right)$ for left-handed participants in experimental condition of bias to the left. Left column contains data from dominant hand, right column from the nondominant one. Upper row of each subfigure contains kinks to the left, lower row kinks to the right, respectively. Columns of the subfigures indicate sessions. Kinks to the right have to be understood cum grano due to their sparseness in this experimental condition (see Section 6.4.1). (MG, RW) 


\section{A.2 Technical details of the SensAble Phantom 3.0 6DoF}

The SensAble Phantom 3.0 6DoF is a high-performance that tracks specific point at the handle and also handle orientation with a very high precision. Besides research applications it can also be utilised for teleoperation or virtual assembly or prototyping.

The exact size $^{1}$ of its translational workspace is $838 \times 584 \times 406 \mathrm{~mm}$. Its rotational workspace of the handle covers 297 degrees (yaw), 260 degrees (pitch), and 335 degrees (roll). The nominal position resolutions are $0.02 \mathrm{~mm}$ in translations, 0.0023 degrees in pitch and yaw, and 0.0080 degrees in roll. It can exert peak forces up to $22 \mathrm{~N}$. Continuous exertable forces are only guaranteed up to $3 \mathrm{~N}$. Practically it turned out that forces up to $5 \mathrm{~N}$ work reliable for applications in motor control studies with human probands.

Operated with the OpenHaptics toolkit the machine can be controlled using two operation modes: HD and HL. HD denotes "device level". The user has rather direct, i.e. low-level, control of position tracking and force exertion. HL indicates the "high-level" operation mode. This mode provides a number of functions for comfortable interaction with the device.

\section{A.3 Toolbox for stiffness data analysis}

The toolbox for stiffness data analysis provides modular MATLAB implementations of all methods for raw data analysis from Section 2.2.2, reliable stiffness estimation from Section 2.2.3, and visualisation and characterisation of endeffector stiffness from Section 2.2.4.

The following functions comprise the main functionality, and can be used for a basic analysis:

- function getDataSmoothProjThres( dataFileStart, resultFile, wdh, rampTime )

Processes all files that begin with dataFileStart ${ }^{2}$ and were generated in a recording block of wdh repetitions of the twelve displacing forces

\footnotetext{
${ }^{1}$ All technical data is taken from SensAble (2006).

${ }^{2}$ All displacement files are labelled according to the applied force forceNum and the number repNum of the repetition of this force in the individual block according to dataFileStart.forceNum.repNum.
} 
which were ramped on within rampTime ms. Endeffector velocity is projected onto the displacing force vector for the detection of the holding phase. The resulting displacement for each repetition of each of the forces is logged into the resultFile along with the force vector.

- function leaveOutForSigma( datenDat, anz, resultFile )

Applies the $N$-fold cross-validation procedure (with anz repetitions) from Section 2.3.2 to the force-displacement data from datenDat (a results file of the previous function). The resulting stiffnesses are stored in the resultFile for statistical inspection.

- function list_of_K = lnoStiffness $(\mathrm{n}, \mathrm{m}$, datenDat, resultFile)

This function directly applies the $N$-fold cross-validation procedure (with $\mathrm{n}$ repetitions of drawing data samples of size $\mathrm{m}$ ) to the force-displacement data from datenDat, and stores the best stiffness estimate along with the estimated variance in the resultFile.

- function Kreturn=findStiffnessWithSigma( stiffnessLNOfile )

Finds the best stiffness estimate for the stiffnessLNOfile which was generated by leaveOutForSigma.

- function drawStiffnessEllipsoid( K, pos )

Visually displays the symmetric part of the stiffness maxtrix $\mathrm{K}$ in the current MATLAB plot at the position that is defined by the three components of the vector pos.

Their MATLAB documentation ${ }^{3}$ provides further links to and instructions for additional functions that are highly configurable.

\section{A.4 Toolbox for timed via-point movements}

\section{A.4.1 Script language for specification of experiments}

The listing in Table A.3 shows the definition of a protocol for an experimental session. Predefined high-level types for via-point movements with a starting

\footnotetext{
3 help nameOfFunction
} 
position, one intermediate target, one obstacle and a final movement target behind the obstacle are

- FirstExperiment,

- ProbabilityExperiment,

- ProbabilityDelay, and

- ProbabilityScaleDelay ${ }^{4}$.

Setup parameters (cf. Table A.4) can be changed by setting the according setup values. Further session parameters are available in Table A.5, and refer to specific functionality of the Simulator.

The Boolean PopUp parameter allows to hide the position of the final movement target until the participant's hand reaches the intermediate target. In ProbabilityScaleDelay experiments the revealing of the final movement target is done upon arrival in a hidden intermediate target at a configurable fraction of hiddenTargetHeight of the distance between starting position and the first movement target. The startPositionAngle allows to control the angle between starting position and first movement target T1. -90 means approaching T1 from the left, 90 from the right, respectively.

In the combinator TYPE1 each value of parameter one is combined with all of parameter two before taking he next value. TYPE2 applies the same strategy the other way around for parameter two and parameter one. The combinator MIX changes both parameters at the same time.

The generators UP, DOWN, and RANDOM work as the names suggest by increasing or decreasing the value or picking up a random one. The generator OUTSIDE_IN generates a sequence from an interval of indices by starting at one end of the interval, picking the value, jumping to the other end, picking the value, jumping back to the previous end, picking the next value that has not been taken before, and so on. So $\{1,2,3,4\}$ transforms to $\{1,4,2,3\}$. INSIDE_OUT works the other way around, starting in the middle of the sequence. ${ }^{5}$

All session definitions have to start with the keyword new followed by the specification of the Experiment in the next line. The keyword end indicates the

\footnotetext{
${ }^{4}$ The low-level types BallsAndWalls and BasicProbability were only introduced for sake of structure, and provide important functionality for the types of experiments in subsequent rows of the listing.

${ }^{5}$ If the middle falls between two values, the one further to the right is taken.
} 
end of the session definition. One protocol file has to contain contain at least one sessions.

Furthermore, all experiment types (using their default values) are accessible through the graphical user interface which can be used upon a right-click on the screen.

\section{A.4.2 Hierarchy of experimental types and their default settings}

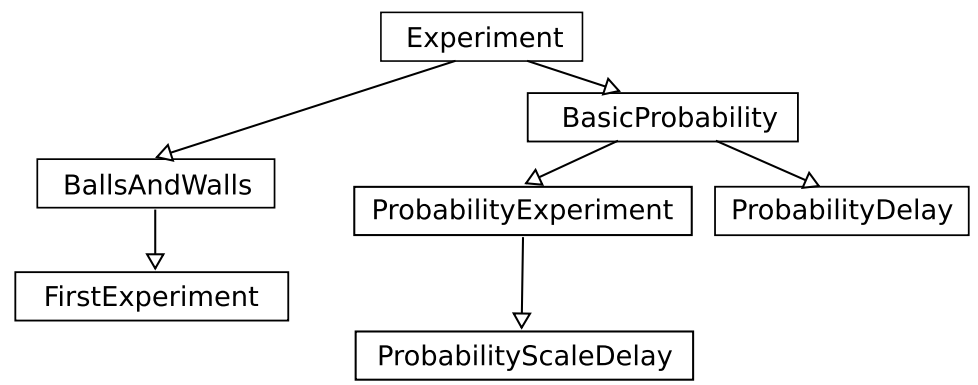

Figure A.17: UML overview of relation between preconfigured types of experiments.

Figure A.17 shows a coarse overview of available tpes of experiments. Data fields and methods are ommited for sake of clearness. The class BallAndWall provides basic functionality that can be used to display almost arbitrary settings of movement targets (balls) and obstacles (walls). FirstExperiment implements an example setup which also was used to give participants the time to accomodate with the sstem before starting the actual experiment.

Basic functionality of handling timing and frequency of occurance of obstacles and movement targets is provided by the BasicProbability. In addition ProbabilityExperiment and ProbabilityDelay provide implementations of specific options for efficient fine-tuning of specific experiments. Technical details on ProbabilityExperiment are given in Section 4.3.1. Table A.5 gives an overview of experimental parameters that can be used for designing specific setups. 
Table A.3: Left: Protocol used for training sessions. Param1, Param2, Repetitions, and Combinator are just mentioned for sake of illustration here. They contained the default values. Target T2 was shown from the beginning of a trial on. Right: Protocol used for experiment. Setup 3 specifies the probability of catch trials with T2 in the centre behind the obstacle. Setup 6 defines the number of repetitions for a single session. Setup 10 is used for setting the tolerance time in which the region of T2 has to be left. The ProbabilityExperiment by default reveals the location of T2 when reaching $\mathrm{T} 1$.

$\begin{array}{llll}\text { new } & & \text { new } & \\ \text { Experiment } & \text { FirstExperiment } & \text { Experiment } & \text { ProbabilityExperiment } \\ \text { Repetitions } & 1 & \text { Param1 } & \text { probability } \\ \text { Param1 } & \text { xpos } & \text { Range1 } & 0.50 .5 \\ \text { Generator1 } & \text { SHUFFLE } & \text { Resolution1 } & 1 \\ \text { Resolution1 } & 2 & \text { Repetitions } & 1 \\ \text { Range1 } & -0.80 .8 & \text { Param2 } & \text { angle } \\ \text { Param2 } & \text { angle } & \text { Range2 } & 00 \\ \text { Generator2 } & \text { SHUFFE } & \text { Resolution2 } & 1 \\ \text { Resolution2 } & 5 & \text { Setup } 3 & 0.1 \\ \text { Range2 } & -6060 & \text { Setup } 6 & 250 \\ \text { Combinator } & \text { MIX } & \text { Setup } 10 & 0.35 \\ \text { end } & & \text { end } & \end{array}$

Table A.4: Overview over experimental parameters and their default values. Restrictions upon parameters were set for practical purposes, and have to be obeyed during definition. Violations lead to abortion of the session.

\begin{tabular}{|c|c|c|c|}
\hline Class & ParamIndex & Parameter & Restriction \\
\hline BallsAndWalls & 1 & centerWallLength & ] $0,2[$ \\
& 2 & TargetSize & ] $0,1[$ \\
\hline FirstExperiment & 3 & PopUp & 0,1 \\
& 4 & repeatOvershoots & 0,1 \\
\hline BasicProbability & 3 & neutralProbability & {$[0,1]$} \\
& 4 & mean targetPosition & ]$-1,1[$ \\
& 5 & mean probability & ]$-1,1[$ \\
& 6 & repetitions & ] $0,1000[$ \\
\hline ProbabilityDelay & 10 & timeTolerance & \\
\hline ProbabilityScaleDelay & 11 & startPositionAngle & {$[-90,90]$} \\
\hline
\end{tabular}


Table A.5: Overview over further experimental parameters.

\begin{tabular}{|l|l|l|}
\hline Name & Description & Values \\
\hline Param [1/2] & first/second varying parameter & $\begin{array}{l}\text { xpos, angle } \\
\text { probability }\end{array}$ \\
\hline Range [1/2] & $\begin{array}{l}\text { lower and upper limit of } \\
\text { range of first/second parameter }\end{array}$ & $\begin{array}{l}\text { double (checked } \\
\text { for consistency) }\end{array}$ \\
\hline Resolution [1/2] & $\begin{array}{l}\text { number of (equally spaced) values } \\
\text { of parameter range }\end{array}$ & integer \\
\hline Generator [1/2] & how parameter range is parsed & $\begin{array}{l}\text { UP, DOWN, SHUFFLE } \\
\text { INSIDE_OUT } \\
\text { OUTSIDE_IN }\end{array}$ \\
\hline Combinator & $\begin{array}{l}\text { combination method for both } \\
\text { prameters to form pairs of trials }\end{array}$ & $\begin{array}{l}\text { TYPE1, TYPE2, } \\
\text { MIX }\end{array}$ \\
\hline
\end{tabular}




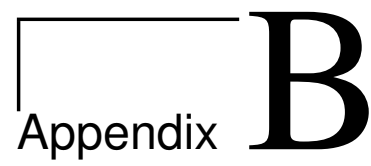

\section{List of own publications}

Fiedler (2007) School on Cognition and Action (Poster)

Fiedler et al. (2008) Computational Neuroscience Meeting (Poster)

Martius et al. (2008) IEEE IROS (Paper)

Fiedler and Herrmann (2008) GGNB Opening Ceremony (Poster )

Fiedler (2009) Göttingen Meeting of the German Neuroscience Society (Poster)

Fiedler and Herrmann (2009b) Conference on Progress in Motor Control (Poster)

Fiedler and Herrmann (2009a) GGNB Science Day (Poster)

Fiedler et al. (2010) Bernstein Conference on Computational Neuroscience (Poster)

Fiedler and Herrmann (2011) Computational Neuroscience Meeting (Poster, Conference Paper) 


\section{Bibliography}

Acosta, A. M., Kirsch, R. F., and Perreault, E. J. (2000). A robotic manipulator for the characterization of two-dimensional dynamic stiffness using stochastic displacement perturbations. J Neurosci Meth, 102:177-186. 6, 9, 104, 106

Arbib, M. A. (1975). Artificial intelligence and brain theory: Unities and diversities. Ann Biomed Eng, 3:238-274. 50

Archambault, P. S., Ferrari-Toniolo, S., and Battaglia-Mayer, A. (2011). Online control of hand trajectory and evolution of motor intention in the parietofrontal system. J Neurosci, 31(2):742-752. 50

Biess, A., Liebermann, D. G., and Flash, T. (2007). A computational model for redundant human $3 \mathrm{~d}$ pointing movements: Integration of independent spatial and temporal motor plans simplifies movement dynamics. J Neurosci, 27(48):13045-13064. 17, 38

Biryukova, E., Roschin, V., Frolov, A., Ioffe, M., Massion, J., and Dufosse, M. (1999). Forearm postural control during unloading: anticipatory changes in elbow stiffness. Exp Brain Res, 124:107-117. 12

Bizzi, E., Accornero, N., Chapple, W., and Hogan, N. (1984). Posture control and trajectory formation during arm movement. J Neurosci, 4:2738-2744. 5, 8,104

Breteler, M. D. K., Hondzinski, J. M., and Flanders, M. (2003). Drawing sequences of segments in 3d: Kinetic influences on arm configuration. $J$ Neurophysiol, 89:3253-3263. 50 
Buneo, C. A., Soechting, J. F., and Flanders, M. (1997). Postural dependence of muscle actions: Implications for neural control. J Neurosci, 17(6):2128-2142. 7

Cisek, P. and Kalaska, J. F. (2010). Neural mechanisms for interacting with a world full of action choices. Annu Rev Neurosci, 33:269-298. 73

Darainy, M., Malfait, N., Gribble, P. L., Towhidkhah, F., and Ostry, D. J. (2004). Learning to control arm stiffness under static conditions. J Neurophysiol, 92:3344-3350. 7

Deco, G. and Rolls, E. (2005). Attention, short-term memory, and action selection: a unifying theory. Prog Neurobiol, 76(4):236-256. 73

Demiris, J. and Hayes, G. M. (1999). Active and passive routes to imitation. In Proceedings of the AISB Symposium on Imitation in Animals and Artifacts. 50

Diftler, M., Mehling, J., Abdallah, M., Radford, N., Bridgwater, L., Sanders, A., Askew, R., Linn, D., Yamokoski, J., Permenter, F., Hargrave, B., Platt, R., Savely, R., and Ambrose, R. (2011). Robonaut 2: The first humanoid robot in space. Proc IEEE ICRA. 1

Eckmann, J. P., Kamphorst, S. O., and Ruelle, D. (1987). Recurrence plots of dynamical systems. Europhys Let, 5:973-977. 87

Feldman, A. G. (1966). Functional tuning of the nervous system with control of movement or maintenance of a steady posture. 2. controllable parameters of the muscle. Biophysics, 11:565-578. 5, 6, 8, 104

Fiedler, K. (2007). Human arm stiffness in three-dimensional space. In Book of abstracts of the 5th Neuro-IT and Neuroengineering School on Cognition and Action. 3, 140

Fiedler, K. (2009). Arm stiffness and movement control. In Book of abstracts of the 8th Goettingen Meeting of the German Neuroscience Society. 140

Fiedler, K. and Herrmann, J. M. (2008). Human arm stiffness and movement control. Technical report, GGNB (Opening ceremony). 140

Fiedler, K. and Herrmann, J. M. (2009a). Human arm stiffness and movement control. Technical report, GGNB (Science Day). 140 
Fiedler, K. and Herrmann, J. M. (2009b). On the relation between arm stiffness and movement control. In Book of abstracts of the Seventh Conference on Progress in Motor Control. 3, 140

Fiedler, K. and Herrmann, J. M. (2011). Nonlinear integration of evidence in a dynamic motor task. Proceedings Twentieth Annual Computational Neuroscience Meeting CNS*2011. 3, 140

Fiedler, K., Martius, G., Hesse, F., and Herrmann, J. M. (2008). Structured control from self-organizing arm movements. In Book of abstracts of Seventeenth Annual Computational Neuroscience Meeting. 3, 140

Fiedler, K., Solla, S., and Herrmann, J. M. (2010). A nonlinear model for probability estimation and revision in a human motor task. In Book of abstracts of the 6th Bernstein Conference on Computational Neuroscience. 3, 4, 73, 140

Finley, R. F., Wirta, R. W., and Cody, K. (1968). Muscle synergies in motor performance. Arch Phys Med Rehabil, 49:655-660. 50

Fishbach, A., Roy, S. A., Bastianen, C., Miller, L. E., and Houk, J. C. (2005). Kinematic properties of on-line error corrections in the monkey. Exp Brain Res, 164(4):442-457. 51

Flash, T. and Gurevich, I. (1991). Arm stiffness and movement adaptation to external loads. Proc IEEE Eng Med Biol Soc, 13:885-886. 6, 29, 30, 107

Flash, T. and Hochner, B. (2005). Motor primitives in vertebrates and invertebrates. Curr Opin Neurobiol, 15:660-666. 49, 50

Flash, T. and Hogan, N. (1985). The coordination of arm movements: an experimentally confirmed mathematical model. J Neurosci, 5:1688-1703. 50, 53

Flash, T. and Mussa-Ivaldi, F. A. (1990). Human arm stiffness characteristics during the maintenance of posture. Exp Brain Res, 82(2):315-326. iii, 6, 18, 19, 20, 106

Foisy, M. and Feldman, A. G. (2006). Threshold control of arm posture and movement adaptation to load. Exp Brain Res, 175(4):726-744. 16

Franklin, D. F., Liaw, G., Milner, T. E., Osu, R., Burdet, E., and Kawato, M. (2007). Endpoint stiffness of the arm is directionally tuned to instability in the environment. J Neurosci, 27(29):7705-7716. 1, 7, 9, 106 
Franklin, D. W. and Milner, T. E. (2003). Adaptive control of stiffness to stabilize hand position with large loads. Exp Brain Res, 152:211-220. 7

Freedman, D. A. and Peters, S. C. (1984). Bootstrapping a regression equation: Some empirical results. JASA, 79(385):97-106. 22

Goble, D. J. and Brown, S. H. (2008a). The biological and behavioral basis of upper limb asymmetries in sensorimotor performance. Neurosci Biobehav Rev, 32:598-610. 63

Goble, D. J. and Brown, S. H. (2008b). Upper limb asymmetries in the matching of proprioceptive versus visual targets. J Neurophysiol, 99:3063-3074. 76, 77

Gomi, H. (2008). Implicit online corrections of reaching movements. Curr Opin Neurobiol, 18(6):558-564. 51

Gomi, H. and Kawato, M. (1996). Equilibrium-point control hypothesis examined by measured arm stiffness during multijoint movement. Science, 272:117120. $7,9,104$

Gomi, H. and Osu, R. (1998). Task-dependent viscoelasticity of human multijoint arm and its spatial characteristics for interaction with environments. $J$ Neurosci, 18(21):8965-8978. 1, 7

Henis, E. A. and Flash, T. (1995). Mechanisms underlying the generation of averaged modified trajectories. Biol Cybern, 72:407-419. 53

Hogan, N. (1985). The mechanics of multi-joint posture and movement. Biol Cybern, 52:315-331. 5

Honda, H. (1982). Rightward superiority of eye movements in a bimanual aiming task. Q J Exp Psychol A, 34(4):499-513. 76

Ijspeert, A. (2001). A connectionist central pattern generator for the aquatic and terrestrial gaits of a simulated salamander. Biol Cybern, 84(5):331-348. 108

Ketchama, C. J., Dounskaiaa, N. V., and Stelmach, G. E. (2004). Multijoint movement control: the importance of interactive torques. Prog Brain Res, 143:207-218. 8

Koerding, K. P. and Wolpert, D. M. (2006). Bayesian decision theory in sensorimotor control. Trends Cogn Sci, 10(7):319-326. 73, 76 
Krutky, M. A., Ravichandran, V. J., Trumbower, R. D., and Perreault, E. J. (2010). Interactions between limb and environmental mechanics influence stretch reflex sensitivity in the human arm. J Neurophysiol, 103(1):429-440. 38,106

Kurtzer, I., Pruszynski, J. A., and Scott, S. H. (2009). Long-latency responses during reaching account for the mechanical interaction between the shoulder and elbow joints. J Neurophysiol, 102:3004-3015.

Manoonpong, P., Geng, T., Kulvicius, T., Porr, B., and Woergoetter, F. (2007). Adaptive, fast walking in a biped robot under neuronal control and learning. PLoS Comput Biol, 3(7):e134. 108

Martius, G., Fiedler, K., and Herrmann, J. M. (2008). Structure from behaviour in autonomous agents. IEEE IROS: Proceedings, 1:858-862. 140

Mataric, M. J. (2000). Sensory-motor primitives as a basis for imitation: Linking perception to action and biology to robotics. In Imitation in Animals and Artifacts, pages 391-422. MIT Press. 50

Mattia, M., Ferraina, S., and Giudice, P. D. (2010). Dissociated multi-unit activity and local field potentials: A theory inspired analysis of a motor decision task. NeuroImage, 52:812-823. 75

McIntyre, J., Mussa-Ivaldi, F., and Bizzi, E. (1996). The control of stable postures in the multijoint arm. Exp Brain Res, 110:248-264. 16

Migliore, S. A., Brown, E. A., and DeWeerth, S. P. (2005). Biologically inspired joint stiffness control. Proceedings of ICRA, 1:4508-4513. 1, 8

Mihaltchev, P., Archambault, P. S., Feldman, A. G., and Levin, M. F. (2005). Control of double-joint arm posture in aldults with unilateral brain damage. Exp Brain Res, 163:468-486. 16

Mussa-Ivaldi, F. A. and Bizzi, E. (2000). Motor learning through the combination of primitives. Phil Trans $R$ Soc Lond, 355:1755-1769. 50

Mussa-Ivaldi, F. A., Gister, S., and Bizzi, E. (1993). Convergent force fields organized in the frogs spinal cord. J Neurosci, 13(2):467-491. 5

Mussa-Ivaldi, F. A., Gister, S., and Bizzi, E. (1994). Linear combinations of primitives in vertebrate motor control. Proc Natl Acad Sci, 91:7534-7538. 50 
Mussa-Ivaldi, F. A., Hogan, N., and Bizzi, E. (1985). Neural, mechanical and geometric factors subserving arm posture in humans. J Neurosci, 5:27322743. $8,12,18,28,104,106$

Nigg, B. M., MacIntosh, B. R., and Mester, J. (2000). Biomechanics and biology of movement. Human Kinetics. 110

Osu, R. and Kawato, H. (1999). Multijoint muscle regulation mechanisms examined by measured human arm stiffness and emg signals. J Neurophysiol, 81:1458-1468. 7

Perreault, E. J., Kirsch, R. F., and Acosta, A. M. (1999). Multiple-input, multiple-output system identification for characterization of limb stiffness dynamics. Biol Cybern, 80:327-337. 9

Pisella, L., Grea, H., Vighetto, A., Desmurget, M., and Rode, G. (2000). An 'automatic pilot' for the hand in human posterior parietal cortex: Towards reinterpreting optic ataxia. Nat Neurosci, 3(7):729-736. 49, 51, 63, 72, 113

Plamondon, R., Alimi, A. M., Yergeau, P., and Leelerc, F. (1993). Modelling velocity profiles of rapid movements: a comparative study. Biol Cybern, 69:119128. $49,53,61,113$

Pruszynski, J. A., Kurtzer, I., and Scott, S. H. (2008). Rapid motor responses are appropriately tuned to the metrics of a visuospatial task. $J$ Neurophysiol, 100:224-238. 9

Pruszynski, J. A., Lillicrap, T. P., and Scott, S. H. (2010). Complex spatiotemporal tuning in human upper-limb muscles. J Neurophysiol, 103:564 - 572 . 50

Rack, P. and Westbury, D. R. (1969). The effects of length and stimulus rate on tension in isometric cat soleus muscle. J Physiol (Lond), 204:443-460. 7

Rosenblatt, F. (1958). The perceptron: A probabilistic model for information storage and organization in the brain. Psychol Rev, 65(6):386-408. 55

Sainburg, R. L. (2002). Evidence for a dynamic-dominance hypothesis of handedness. Exp Brain Res, 142(2):241-258. 76

Sanger, T. D. (2000). Human arm movements described by a low-dimensional superposition of principal components. J Neurosci, 20(3):1066-1072. 8 
SensAble (2006). Specifications for the phantom premium 3.0/6dof haptic device. Technical report, SensAble Technologies, Inc. 134

Shadmehr, R. and Wise, S. P. (2005). The computational neurobiology of reaching and pointing: A foundation for motor learning. MIT Press.

Soechting, J. F., Buneo, C. A., Herrman, U., and Flanders, M. (1995). Moving effortlessly in three dimensions: Does donders' law apply to arm movement? J Neurosci, 15(9):6271-6280. 8

Soon, C. S., Brass, M., Heinze, H.-J., and Haynes, J.-D. (2008). Unconscious determinants of free decisions in the human brain. Nat Neurosci, 11:543-545. 75

Sosnik, R., Hauptmann, B., Karni, A., and Flash, T. (2004). When practice leads to co-articulation: the evolution of geometrically defined movement primitives. Exp Brain Res, 156:422-438. 62

Tee, K. P., Burdet, E., Chew, C. M., and Milner, T. E. (2004). A model of force and impedance in human arm movements. Biol Cybern, 90:368-375. 17

Tillery, S. I. H., Ebner, T. J., and Soechting, J. F. (1995). Task dependence of primate arm posture. Exp Brain Res, 104:1-11. 25

Torres, E. B. and Zipser, D. (2002). Reaching to grasp with a multi-jointed arm. i. computational model. J Neurophysiol, 88:2355-2367. 62

Torres, E. B. and Zipser, D. (2004). Simultaneous control of hand displacements and rotations in orientation-matching experiments. J Appl Physiol, 96:19781987. 62

Trommershaeuser, J. (2009). Biases and optimality of sensory-motor and cognitive decisions. Prog Brain Res, 174:267-278. 76

Tsuji, T., Morasso, P. G., Goto, K., and Ito, K. (1995). Human hand impedance characteristics during maintained posture. Biol Cybern, 72:475-485. 6, 7, 8, 104

Westendorff, S., Klaes, C., and Gail, A. (2010). The cortical timeline for deciding on reach motor goals. J Neurosci, 30(15):5426-5436. 75

Winter, D. A. (2005). Biomechanics and motor control of human movement. John Wiley \& Sons, Inc., 3rd edition. 13 
Xu, Y. and Hollerbach, J. M. (1998). Identification of human joint mechanical properties from single trial data. IEEE Trans Biomed Eng, 45(8):1051-1060. 9

Yang, Z., Poo, A. N., and Hong, G. S. (1993). A new method for implementing active stiffness control of robot manipulator by using sliding mode. AsiaPacific Workshop on Advances in Motion Control: Proceedings, 1:165-170. 1, 8 